Endbericht

\title{
Zwischenevaluierung des Förderschwerpunkts
}

Talente 
Diese Studie wurde im Auftrag im Auftrag des Bundesministeriums für Verkehr, Innovation und Technologie (BMVIT) durchgeführt.

VERFASSER/IN DES BERICHTS:

Eva Heckl (Projektleitung)

Sonja Sheikh

Laurenz Wolf

INTERNES REVIEW/BEGUTACHTUNG:

Sonja Sheikh

LAYOUT:

Martina Gugerell

Die vorliegende Studie wurde nach allen Maßstäben der Sorgfalt erstellt.

Die KMU Forschung Austria übernimmt jedoch keine Haftung für Schäden oder Folgeschäden, die auf diese Studie oder auf mögliche fehlerhafte Angaben zurückgehen.

Dieses Werk ist urheberrechtlich geschützt. Jede Art von Nachdruck, Vervielfältigung, Verbreitung, Wiedergabe, Übersetzung oder Einspeicherung und Verwendung in Datenverarbeitungssystemen, und sei es auch nur auszugsweise, ist nur mit ausdrücklicher Zustimmung der KMU Forschung Austria gestattet.

Mitglied bei:

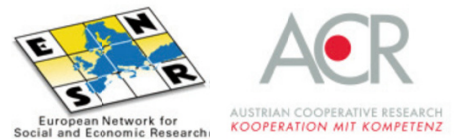




\section{Inhaltsverzeichnis}

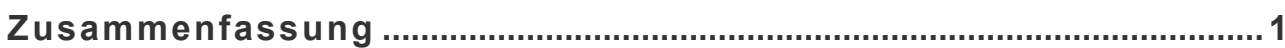

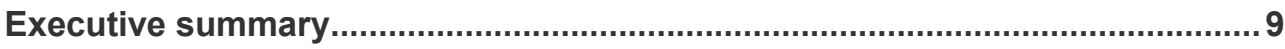

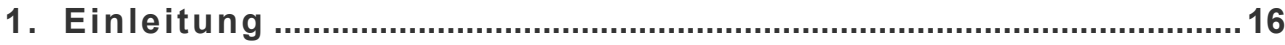

1.1. Gegenstand und Ziele der Evaluierung ..........................................16

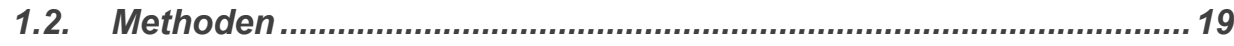

2. Das Konzept des Förderschwerpunkts Talente ..........................21

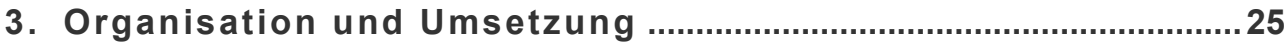

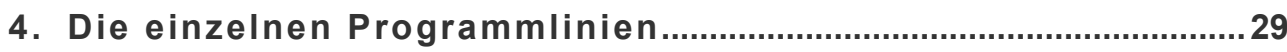

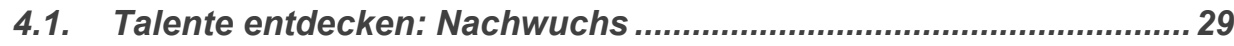

4.1.1. Praktika für SchülerInnen .......................................... 29

4.1.2. Talente regional ........................................................ 35

4.2. Talente nützen: Chancengleichheit ..................................................46

4.2.1. FEMtech Forschungsprojekte .................................... 46

4.2.2. FEMtech Karriere .................................................. 51

4.2.3. FEMtech Praktika für Studentinnen ............................ 57

4.2.4. FEMtech Dissertationen/Dissertationen in den Themen

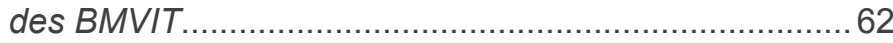

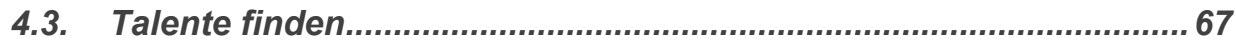

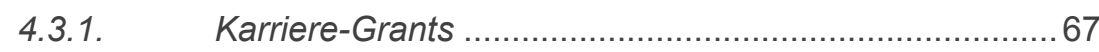

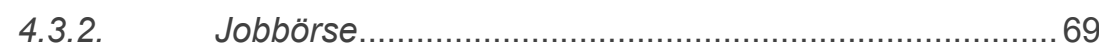

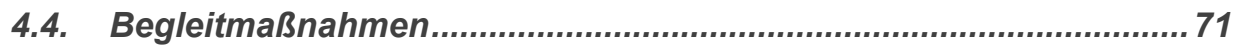

4.4.1. FEMtech Netzwerktreffen............................................ 71

4.4.2. Tickets für Talente@Alpbach ..........................................72

5. Gesamtbetrachtung und Schlussfolgerungen ...........................73

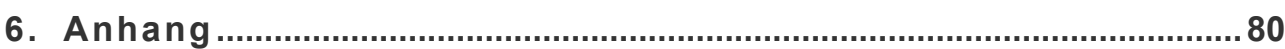

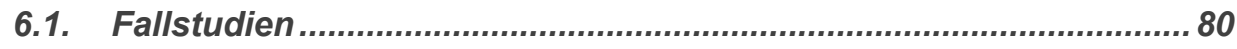

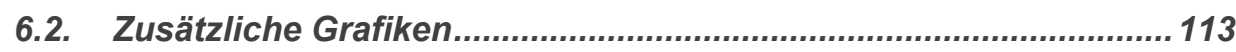




\section{Grafikverzeichnis}

Grafik 1 Die Programmlinien des Forschungsschwerpunkts Talente ............. 22

Grafik 2 Anteil weiblicher bzw. männlicher Praktikumsteilnehmerlnnen ......... 30

Grafik 3 Beurteilung der Praktika durch die SchülerInnen .............................. 32

Grafik 4 Veränderung des Interesses der PraktikantInnen an technisch-naturwissenschaftlichen Berufen

Grafik 5 Wichtigkeit von Faktoren, um Kinder/Jugendliche für Naturwissenschaft/Technik zu begeistern........................................ 39

Grafik 6 Zufriedenheit mit der Kooperation im Rahmen des Talente regional Projekts................................................................... 40

Grafik $7 \quad$ Faktoren für eine gut funktionierende Kooperation ........................... 41

Grafik 8 Wichtigkeit der Faktoren für den Erfolg eines Talente regional

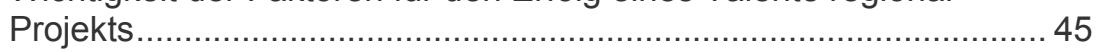

Grafik 9 Additionalität FEMtech Praktika für Studentinnen ........................... 61

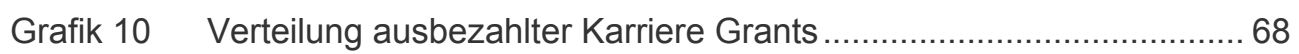

Grafik 11 Gesamtanzahl und Tagesdurchschnitt der veröffentlichten

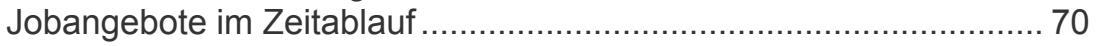

Grafik 12 Verteilung der SchülerInnenpraktika nach Schultypen .................... 113

Grafik 13 Anspruchsgehalt des Praktikums ................................................... 113

Grafik 14 Initiative zur Teilnahme am Projekt ............................................ 114

Grafik 15 Kenntnis der Programmlinie Talente regional ............................... 114

Grafik 16 Früherer Kontakt zu ProjektpartnerInnen ........................................ 115

Grafik 17 Wirkungen bei den teilnehmenden Kindern/Jugendlichen ............... 115

Grafik 18 Wirkungen bei den PädagogInnen ............................................... 116

Grafik 19 Praktikumsdauer der FEMtech Praktika für Studentinnen ............... 116

Grafik 20 Verteilung der Organisationstypen und Projektteilnahmen .............. 117

Grafik 21 Verteilung Erstförderungen ....................................................... 117

\section{Tabellenverzeichnis}

Tabelle 1 Eckpunkte von Praktika für SchülerInnen ....................................... 29

Tabelle 2 Eckpunkte von Talente regional ................................................... 35

Tabelle 3 Eckpunkte von FEMtech Forschungsprojekte.................................. 46

Tabelle 4 Eckpunkte von FEMtech Karriere................................................... 51

Tabelle 5 Eckpunkte von FEMtech Praktika ................................................. 57

Tabelle 6 Eckpunkte von FEMtech Dissertationen/Dissertationen in

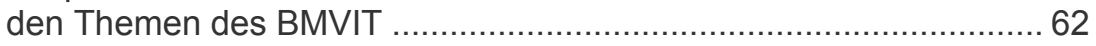

Tabelle 7 Eckpunkte von Karriere Grants .......................................................... 67 


\section{Zusammenfassung}

Der Förderschwerpunkt Talente bündelt alle Aktivitäten zur Förderung der Humanpotenziale im BMVIT (damit wurden Programme wie brainpower austria, FEMtech und generation innovation unter ein Dach zusammengefasst), um die Ausschöpfung des Humanpotenzials im anwendungsorientierten, naturwissenschaftlich-technischen FTI-Bereich zu erhöhen. Der Förderschwerpunkt fokussiert auf den gesamten Karriereverlauf von ForscherInnen im weitesten Sinn, d. h. es werden (potentielle) ForscherInnen im Kindesalter ebenso gefördert wie etablierte ForscherInnen. Der Förderschwerpunkt gliedert sich in drei Interventionsfelder mit insgesamt acht Programmlinien:

- Talente entdecken: Nachwuchs

○ Praktika für Schülerinnen und Schüler

- Talente regional

- Talente nützen: Chancengleichheit

- FEMtech Forschungsprojekte

- FEMtech Karriere

- FEMtech Praktika für Studentinnen

- FEMtech Dissertationen

- Talente finden: Forscherinnen und Forscher

- Karriere-Grants

- Die österreichische Jobbörse für Forschung, Entwicklung und Innovation

Die KMU Forschung Austria wurde beauftragt, die Zwischenevaluierung des Förderschwerpunkts durchzuführen. Ziel der gegenständlichen Evaluierung ist die Analyse und Reflexion des bisherigen Verlaufs des Förderschwerpunkts (Betrachtungszeitraum sind die Jahre 2011 bis 2013), dabei werden die Konzeption, die Umsetzung und die bisher feststellbaren Wirkungen untersucht. Auf Basis der Resultate werden Schlussfolgerungen und Empfehlungen für die Weiterentwicklung des Förderschwerpunkts erarbeitet. Die Evaluierung kombiniert quantitative und qualitative Methoden: Dokumentenanalyse, Interviews mit Stakeholdern, Analyse der FFG-Monitoringdaten, Online-Befragung von in Talente regional Projekten involvierten PädagogInnen, Fallstudien sowie Interviews mit Projektverantwortlichen.

\section{Das Konzept des Förderschwerpunkts Talente}

Der Förderschwerpunkt deckt alle Karrierestationen (Vorschulkinder, SchülerInnen, Studentinnen/DissertantInnen, ForscherInnen) im weitesten Sinn ab, und die verschiedenen Programmlinien greifen ineinander. Jede „Karrierestation“ (mit Ausnahme der Vorschulkinder) wird von mindestens zwei Programmlinien adressiert. 
Als Querschnittsmaterie wird "Gender" zudem in fast allen Programmlinien explizit berücksichtigt. Somit entspricht das Konzept von Talente seinem Ziel, Menschen in der angewandten Forschung über den gesamten Karriereverlauf zu unterstützen.

Der Förderschwerpunkt zeichnet sich zudem durch eine hohe Flexibilität und eine große Auswahl an Instrumenten aus, mit denen unterschiedlichste Zielgruppen erreicht werden können und die auch wichtige Schnittstellen und kritische Phasen im Karriereverlauf (Bewusstseinsbildung bei Kleinkindern, Ausbildungsentscheidungen von Schülerlnnen, Berufsentscheidungen von Studentinnen, Karriereentscheidungen von ForscherInnen) adressieren. Alle Programmlinien mit Ausnahme von Karriere-Grants setzen nicht nur auf die Förderung des Individuums, sondern auch auf die Verbesserung von Rahmenbedingungen, indem Unternehmen und Forschungseinrichtungen als Fördernehmerlnnen für die Förderung von Humanpotenzial sensibilisiert werden.

\section{Organisation und Umsetzung}

Mit dem Förderschwerpunkt Talente und seinem Fokus auf Humanressourcen hat die FFG Aufgaben übernommen, die nicht in ihr Kerngeschäft - die Forschungsförderung im klassischen Sinn - fallen. Auch handelt es sich bei einigen Programmlinien um Kleinstförderungen, was zusätzlich zur Sonderstellung des Förderschwerpunkts innerhalb der FFG beiträgt. Dieses „Besondere“ spiegelt sich auch bei den Fördernehmerlnnen und involvierten Personen wieder. EinzelforscherInnen (bei Karriere-Grants) und ErstfördernehmerInnen (v. a. bei Praktika für Schülerlnnen) sind im Förderschwerpunkt Talente vertreten genauso wie HRAbteilungen (z. B. bei FEMtech Karriere) von Unternehmen. Diese Förderneulinge benötigen intensivere Betreuung, sowohl in der Phase der Antragsstellung als auch im Zuge der Abwicklung.

Grundsätzlich dürfte die Zusammenführung der Vorgängerprogramme aus organisatorischer Sicht gelungen sein. Das gemeinsame Dach unterstützt einen besseren Austausch und eine bessere Vernetzung der FFG-Programmverantwortlichen. Damit können die KundInnen in der Beratung auch auf die anderen Programmlinien verwiesen werden, was der Idee der Förderkette entspricht. Der Wegfall des FEMtech Kompetenzzentrums und der Koordinierungsstelle (generation innovation) und einiger Begleitmaßnahmen beeinträchtigt jedoch im Gegenzug die Sichtbarkeit der Programmlinien.

Die Fördernehmerlnnen zeigen sich in allen näher untersuchten Programmlinien grundsätzlich sehr zufrieden mit der Betreuung durch die FFG. 


\section{Die einzelnen Programmlinien}

Die Programmlinie Praktika für Schülerinnen und Schüler fördert Schülerlnnenpraktika in Unternehmen und Forschungseinrichtungen im Zeitraum Juni bis September, um Jugendlichen die Tätigkeiten in der Forschung im naturwissenschaftlich-technischen Bereich näherzubringen. Im Zeitraum 2011 bis 2013 wurden in Summe 4.337 geförderte (Ferial-)Praktikumsplätze für Schülerlnnen zur Verfügung gestellt, im Zuge der letzten Ausschreibung 1.500. Die Praktika wurden mehrheitlich von männlichen Jugendlichen in Anspruch genommen, der Anteil der teilnehmenden Schülerinnen stieg aber kontinuierlich auf zuletzt $38 \%$ im Jahr 2013. Die PraktikantInnen rekrutieren sich zum überwiegenden Teil aus Berufsbildenden Höheren Schulen (BHS) - vorwiegend HTL. Knapp jeder dritte Praktikumsplatz wurde von AHS-SchülerInnen besetzt. Dies ist wohl $u$. a. darauf zurückzuführen, dass in den HTLs verpflichtende Ferialpraktika vorgesehen sind, während dies bei AHS-SchülerInnen nicht der Fall ist. Die Verteilung der Praktikumsplätze nach Organisationsart zeigt, dass Unternehmen die deutlich größte Gruppe der PraktikumsgeberInnen bilden, gefolgt von den Universitäten und den außeruniversitären Forschungseinrichtungen.

Die Zufriedenheit der PraktikantInnen liegt auf sehr hohem Niveau und ist im Zeitverlauf nahezu konstant. Fast alle Schülerlnnen bewerten das absolvierte Praktikum mit sehr gut oder gut. Der überwiegende Teil der SchülerInnen bewertete die inhaltlichen Anforderungen zudem als (sehr) anspruchsvoll. Mehr als die Hälfte der befragten SchülerInnen gab an, dass ihr Interesse an einem Beruf im technischnaturwissenschaftlichen Bereich auf Grund des Praktikums gestiegen ist. Auch die PraktikumsanbieterInnen zeigen sich sehr zufrieden. Die Unternehmen und Forschungseinrichtungen ziehen nach eigenen Angaben aus dem Anbieten von Praktika konstant hohen, unmittelbaren bzw. langfristigen Nutzen. Das Praktikumsangebot wird in einem Großteil der Fälle durch die Förderung erst ermöglicht.

Talente regional fördert kooperative Projekte, die Kindern und Jugendlichen ermöglichen, sich über einen längeren Zeitraum in räumlicher Nähe mit FTI-Themen zu beschäftigten. Ein Konsortium muss aus mindestens einem/einer wissenschaftlichen PartnerIn und zwei UnternehmenspartnerInnen mit Innovationsbezug bestehen. Zudem müssen mindestens fünf (vor-)schulische Bildungseinrichtungen aus drei von vier Bildungsstufen beteiligt sein. In den ersten beiden Ausschreibungsperioden wurden 11 bzw. 17 Projekte gefördert. Die ProjektpartnerInnen wollten mit ihren Projekten Kinder und Jugendliche an Naturwissenschaft und Technik heranführen, den Kindern und Jugendlichen Berufsmöglichkeiten aufzeigen und ihre (Forschungs-) Tätigkeiten in der jeweiligen Region bekannter machen. Die PädagogInnen sahen v. a. die Möglichkeit, mit wissenschaftlichen Einrichtungen und Unternehmen zu kooperieren. Im Zentrum aller Projekte stand die Begegnung der SchülerInnen und Kleinkinder mit ForscherInnen, meist im Rahmen von Aktionstagen, an denen gemeinsam experimentiert, geforscht, gemessen, analysiert und 
ausprobiert wurde. Den Abschluss des Projekts bildete in den meisten Fällen ein Forschungsfest, bei dem die Kinder und Jugendlichen ihre Projekte einem breiteren Publikum präsentieren konnten.

Die interviewten ProjektpartnerInnen sehen die Programmlinie Talente regional nicht nur sehr geeignet, um Kinder und Jugendliche für Naturwissenschaft und Technik zu begeistern, sondern erachten derartige Förderungen auch als absolut notwendig, um das Interesse der Zielgruppe zu steigern. Als Stärke der Programmlinie werden die Offenheit bezüglich der Themen und Herangehensweisen genannt sowie die Langfristigkeit der Projekte. Von großer Relevanz sind auch die hohe Praxisorientierung sowie die zahlreichen Interaktionsmöglichkeiten von Forscherlnnen und Kindern/Jugendlichen.

Im Zentrum der FEMtech Forschungsprojekte stehen Forschungsvorhaben aus dem Bereich der angewandten Forschung, die genderrelevante Inhalte abhandeln. In den bisherigen zwei Ausschreibungsrunden wurden jeweils acht Projekte gefördert. Die themenoffene Gestaltung der FEMtech Forschungsprojekte drückt sich in den bisherigen Ausschreibungsrunden durch recht heterogene Anwendungsfelder der geförderten FTI-Projekte aus. Bei den untersuchten FördernehmerInnen handelt es sich überwiegend um Unternehmen und Forschungseinrichtungen, die sich im Zuge ihrer Forschungstätigkeiten bereits mit Genderthemen auseinandergesetzt haben. Für die betrachteten Projekte bzw. die forschenden Organisationen gilt, dass ohne Unterstützung durch die FEMtech Forschungsprojekte die Umsetzung auf Grund unzureichender finanzieller Eigenmittel nicht möglich gewesen wäre. Die spezielle Programmlinie trage dazu bei, die Wahrnehmung unterschiedlicher Lebensrealitäten in oftmals männerdominierte Forschungsfelder einzubeziehen. Die Programmlinie weist eine hohe Zufriedenheit der beteiligten Unternehmen und Forschungseinrichtungen auf und eine neuerliche Teilnahme an FEMtech Forschungsprojekte ist für die Befragten durchaus vorstellbar.

FEMtech Karriere unterstützt strukturelle und nachhaltige Maßnahmen, die zur Chancengleichheit von Frauen und Männern in FTI Unternehmen und außeruniversitären Forschungseinrichtungen im naturwissenschaftlich-technischen Bereich führen. Jedes FEMtech Karriere Projekt besteht aus zwei Pflichtmodulen (Aufbau Genderkompetenz und Projektmanagement) und mehreren Wahlmodulen (aus den Bereichen Öffentlichkeitsarbeit, Personalmanagement, Work-Life-Balance sowie Coaching/Mentoring und Karriereentwicklung). Im Beobachtungszeitraum wurden insgesamt acht FEMtech Karriere Projekte beantragt und gefördert. Bei den FördernehmerInnen handelt es sich Großteils um Organisationen, die sich mit dem Genderthema bereits befasst haben bzw. um Organisationen, deren Personalverantwortliche für das Thema sensibilisiert sind. Der Einsicht über die Notwendigkeit von Personalentwicklungsmaßnahmen im Allgemeinen und Maßnahmen zur Erhöhung der Chancengleichheit im Besonderen stehen aber die fehlenden Ressourcen gegenüber, daher scheint erst durch die Förderung die Möglichkeit der Durch- 
führung derartiger Maßnahmen gegeben. Auswirkungen der FEMtech Karriere Projekte sind z. B. die Sensibilisierung für die Genderthematik v. a. der Führungskräfte, Änderungen von Recruitingprozessen, eine Erhöhung der Anteile weiblicher TechnikerInnen, die Einführung flexibler Arbeitszeitmodelle, die Beförderung von Frauen in Führungspositionen etc.

Das Förderangebot FEMtech Praktika für Studentinnen zielt auf die Gewinnung von weiblichen Nachwuchskräften im Themenbereich naturwissenschaftlich-technischer Forschungsfelder ab. Es wurden 234 (2012) bzw. 490 (2013) Praktikumsstellen gefördert, in erster Linie in Forschungseinrichtungen (rd. $60 \%$ ) und Unternehmen (rd. 30 \%). Die Praktikumsdauer kann je nach Bedarf zwischen einem und sechs Monaten liegen, sechsmonatige Praktika werden am häufigsten vergeben. Für fast jede fünfte Studentin führte laut eigenen Angaben das Praktikum nach Abschluss zu einer weiterführenden Anstellung. Das Feedback der Praktikantinnen ist äußerst gut, sowohl in Hinblick auf die Inhalte als auch die Betreuung während des Praktikums. Durch das Praktikum dürfte auch das Interesse der Studentinnen an naturwissenschaftlich-technischer Forschung gestiegen sein, ebenso wie ihr Fachwissen. Auch die FördernehmerInnen sehen die Praktika sehr positiv, zwei Drittel der teilnehmenden Unternehmen und Forschungseinrichtungen profitieren dabei nach eigenen Angaben unmittelbar oder langfristig von den Praktikantinnen. Die Additionalitätswirkungen dürften sehr hoch sein, da fast alle Fördernehmerlnnen angegeben haben, dass sie ohne Förderung keine oder weniger Praktikumsplätze angeboten hätten.

Im Rahmen der Programmlinie FEMtech Dissertationen bzw. Dissertationen in den Themen des BMVIT werden Dissertationsprojekte von Personen (erste Ausschreibung nur Frauen - diese wurde näher untersucht) gefördert, die für die Dauer des Dissertationsprojekts in einem Unternehmen bzw. einer außeruniversitären Forschungseinrichtung angestellt werden. Im Beobachtungszeitraum wurden fünf bzw. 13 Projekte gefördert. Die Motivation zur Teilnahme war eher inhaltlicher Natur und weniger der Aspekt der Förderung einer Dissertantin. Durch die Programmlinie gelingt es, Frauen bei der Höherqualifizierung zu unterstützen bzw. diese dazu zu motivieren. Die Dissertantinnen werden von den geförderten Unternehmen und Forschungseinrichtungen sehr umfassend betreut. Insbesondere innerhalb der geförderten Forschungseinrichtungen scheinen die Dissertantinnen dabei auch sehr gut in die jeweiligen Forschungsteams bzw. die Organisation als solches integriert zu sein. Für Unternehmen dürfte dies hingegen mitunter eine große Herausforderung darstellen. Bei drei der Projektnehmerlnnen ist die entsprechende Dissertantin bereits in Vollzeit bzw. zu 5/6 angestellt, bei den anderen beiden ist eine Weiterbeschäftigung der Dissertantin nach Projektende geplant bzw. grundsätzlich vorstellbar. Damit dürfte diesen Frauen der Einstieg ins Berufsleben auch durch FEMtech Dissertationen erfolgreich geglückt sein. Von den Projektnehmerlnnen wird die Programmlinie durchwegs sehr positiv beurteilt. 
Die Programmlinie Karriere-Grants unterstützt ForscherInnen, die eine Beschäftigung am Forschungsstandort Österreich anstreben. Förderbar sind hierbei Reisekosten für Vorstellungsgespräche (Interview Grants), Umzugskosten bei Stellenantritt (Relocation Grants) und die berufliche Integration des/der Partners/Partnerin (Dual Career Grants). Im Beobachtungszeitraum wurden 29 (2011), 130 (2012) bzw. 90 (2013) Karriere-Grants ausbezahlt, vorwiegend Interview und Relocation Grants.

Die Österreichische Jobbörse für Forschung, Entwicklung und Innovation ist eine frei zugängliche und kostenlos nutzbare Plattform zur Zusammenführung von JobanbieterInnen und -suchenden im F\&E- sowie Innovationsbereich. Ende des Jahres 2013 wurden 1.031 Jobangebote in der Jobbörse angeboten, wobei der Tagesdurchschnitt der angebotenen Stellen bei einem Wert von 333 lag.

\section{Gesamtbetrachtung und Schlussfolgerungen}

Die drei Interventionsfelder mit ihren acht Programmlinien decken alle Karrierestationen im weitesten Sinn ab und die verschiedenen Programmlinien ergänzen einander zu einer „Förderkette“. Von insgesamt knapp 1.200 Organisationen, die Förderungen aus dem Talente-Portfolio in Anspruch genommen haben, sind etwas mehr als die Hälfte Unternehmen, $17 \%$ Universitäten und Fachhochschulen und $8 \%$ außeruniversitäre Forschungseinrichtungen. $12 \%$ der FördernehmerInnen haben zwei oder mehr Programmlinien in Anspruch genommen, und $33 \%$ haben mehrere Förderungen innerhalb einer Programmlinie erhalten. Zudem scheint der Förderschwerpunkt mit seinen teils sehr niederschwelligen Angeboten (v. a. Praktika für SchülerInnen) ein „Einsteigerprogramm“ zu sein. 17 \% der Fördernehmerlnnen haben im Rahmen von Talente das erste Mal eine Förderung bei der FFG in Anspruch genommen. Die Bedeutung der Genderthematik wird durch die Integration des Programms FEMtech, aber auch durch die Vorgabe, Genderkriterien in anderen Programmlinien zu berücksichtigen, unterstrichen, ebenso wie durch FEMtech Forschungsprojekte, das als einzige Programmlinie nicht die Förderung von Humanressourcen zum Ziel hat, sondern genderrelevante Forschungsprojekte fördert.

Mögliche Interventionsfelder in den einzelnen Programmlinien lassen sich wie folgt resümieren:

- Praktika für Schülerinnen und Schüler: Verstärktes Augenmerk sollte auf Schülerlnnen gerichtet werden, deren Weg noch nicht in Richtung eines naturwissenschaftlich-technischen Studiums oder eines Berufs in diesem Bereich vorgezeichnet ist. Daher sollte die Informationsverbreitung in den AHS verstärkt werden. Die verstärkte Nutzung der Praktikabörse könnte auch zu mehr Ausgewogenheit in Bezug auf den schulischen Hintergrund der PraktikantInnen beitragen. 
- Talente regional: Aufgrund der besonderen Herausforderungen bei der Koordinierung der vielen ProjektpartnerInnen wird eine Erhöhung des Budgets angeregt bzw. die Möglichkeit dem Projektmanagement mehr Budgetanteil zuordnen zu können. Bei der Einbeziehung der BildungspartnerInnen könnte eine Reduzierung auf zwei Bildungsstufen ein solches Projekt vereinfachen (auch bezüglich der Akquise der Bildungspartnerlnnen) und zu noch passgenaueren Angeboten führen. Der Zeitpunkt der Ausschreibung bzw. der Förderentscheidung sollte so gewählt werden, dass die Projekte von Beginn an in der Planung des neuen Schuljahres berücksichtigt werden können.

- FEMtech Forschungsprojekte: Obwohl es sich hier um klassische Forschungsförderung handelt, hat die Programmlinie insofern ihren Platz im Talente-Portfolio, als von einer ausstrahlenden Wirkung der Inhalte auf das Forschungsfeld und die Organisationen, die dieses Feld bearbeiten, ausgegangen werden kann. Solange genderrelevante Forschungsinhalte nicht in ausreichendem Maße in anderen Programmlinien Berücksichtigung finden, scheint eine derartige Programmlinie im Rahmen von Talente unabdingbar.

- FEMtech Karriere: Um einen breiteren Adressatenkreis ansprechen zu können, könnte die Programmlinie adaptiert bzw. gesplittet werden. FEMtech Karriere „light“ könnte erste Maßnahmen zur Implementierung von Chancengleichheit fördern. Hier könnte z. B. nur ein Modul gefördert werden sowie die Förderung (und der Eigenmittelanteil) entsprechend geringer ausfallen. Dies würde die Barrieren für Neueinsteiger bzw. KMU verringern. „Fortgeschrittene“ Fördernehmerlnnen könnten wie bisher ein volles FEMtech Karriere Projekt beantragen, bei einem Folgeantrag müsste dann aber gesichert sein, dass neue Elemente mit neuem Fokus ins Projekt integriert werden.

- FEMtech Praktika für Studentinnen: Da die Studentinnen durch die einschlägige Studienwahl schon einen Schritt in Richtung Naturwissenschaft/Technik gemacht haben, aber die endgültige Berufswahl noch aussteht, scheinen Faktoren, die die Motivation im Berufsfeld zu bleiben erhöhen, wie dies ein Praktikum darstellt, entscheidend, um dem Phänomen der leaky pipeline (Frauen gehen im Karriereverlauf der Forschung verloren) zu begegnen. Daher sollte eine Aufstockung der Mittel für die FEMtech Praktika für Studentinnen in Erwägung gezogen werden.

- FEMtech Dissertationen: Es müsste geprüft werden, in wie weit eine Anhebung der Förderquote für Unternehmen, die erstmals eine Dissertantin / einen Dissertanten aufnehmen bzw. für KMU sinnvoll bzw. notwendig sein könnte, da diese oftmals über wenig Erfahrung in der Durchführung wissenschaftlicher Projekte verfügen und somit einen deutlich höheren Betreuungs- und Organisationsaufwand haben, als Forschungseinrichtungen. 
Insgesamt scheint die Bündelung der Aktivitäten des BMVIT im Bereich Humanressourcen gelungen und notwendig, um die Wichtigkeit des Themas für die Forschung an sich zu unterstreichen, denn es gilt, dass es ohne exzellente Forscherlnnen auch keine exzellente Forschung geben kann. Zur Sichtbarmachung des Förderschwerpunkts Talente und um eine noch größere Breitenwirksamkeit zu erzeugen, erscheint es wichtig, Talente als Marke stärker zu etablieren und den Förderschwerpunkt in seiner Gesamtheit sowie die einzelnen Programmlinien entsprechend zu promoten. 


\section{Executive summary}

The programme (Förderschwerpunkt) Talente pools all activities to support human potential of the Austrian Ministry for Transport, Innovation and Technology (as an umbrella for former programmes like brainpower Austria, FEMtech and generation innovation) to increase the use of human potential in the application-oriented, scientific-technological field of RTI. The programme focusses on the entire career of researchers in the broadest sense, i.e. (potential) researchers - children and pupils - as well as established researchers and can be divided into three intervention fields with in total eight sub-programmes:

- Talente entdecken: Nachwuchs (find talents: young talents)

- Praktika für Schülerinnen und Schüler (internships for pupils)

- Talente regional (talents regional)

- Talente nützen: Chancengleichheit (use talents: equal opportunities)

- FEMtech Forschungsprojekte (research projects)

- FEMtech Karriere (career)

- FEMtech Praktika für Studentinnen (internships for female students)

- FEMtech Dissertationen (doctoral theses)

- Talente finden: Forscherinnen und Forscher (find talents: researchers)

- Karriere-Grants (career-grants)

- Die österreichische Jobbörse für Forschung, Entwicklung und Innovation (the Austrian job platform for research, development and innovation)

The Austrian Institute for SME Research was commissioned with the interim evaluation of the programme. The aim of the evaluation is the analysis of the development of the programme so far (period under review: 2011 - 2013), its concept, implementation and impacts. Conclusions and recommendations for future developments are formulated. The evaluation combines quantitative and qualitative methods: document analysis, interviews with stakeholders, analysis of the monitoring data of the Austrian Research Promotion Agency (FFG), an online survey among teachers involved in Talente regional projects, case studies as well as interviews with project managers.

\section{The concept of the programme Talente}

The programme covers all career levels in the broadest sense (pre-schoolers, pupils, students/postgraduates, researchers) and the various sub-programmes are interlinked. Each "career station" (except pre-schoolers) is addressed by at least two sub-programmes. "Gender" as a cross-cutting issue is explicitly considered in almost all sub-programmes. Thus, the concept corresponds with the aim to support persons in applied research during their entire career. 
The programme is characterised by high flexibility and a large variety of instruments to reach the different target groups and to address also important interfaces and critical periods during the career (raising awareness in small children, educational decisions of pupils, decisions of students regarding career choices, and career decisions of researchers). All sub-programmes except Karriere-Grants do not only focus on the support of individuals but also on the improvement of the framework conditions by sensitising the programme's beneficiaries (enterprises and research institutions) for the support of human potential.

\section{Organisation and implementation}

The Austrian Research Promotion Agency (FFG) resumed responsibility with the programme Talente, which does not belong to its core activities - namely the classical research funding. Furthermore, some of the sub-programmes can be regarded as diminutive funding, which underlines the special position of the programme within the Austrian Research Promotion Agency. This is also reflected in the beneficiaries and the involved persons. Individual researchers (Karriere-Grants) and unexperienced beneficiaries (especially Praktika für Schülerlnnen) participate but also HR departments of enterprises (e.g. FEMtech Karriere). These newcomers regarding funding need more intensive support during the application period and the whole funding process.

From an organisational point of view the merging of the former programmes seems to have been successful in general. The programme as umbrella supports the exchange and networking of the persons responsible for the sub-programmes. Customers can thus be referred to other programmes during the consulting which underlines the idea of the funding chain. Due to the abolition of the FEMtech competence centre and the coordination point (generation innovation) and some other accompanying measures the visibility of the programmes is in the contrary less distinctive.

In all programmes that were analysed the beneficiaries basically confirm being very satisfied with the service of the Austrian Research Promotion Agency (FFG).

\section{The sub-programmes}

The sub-programme Praktika für Schülerinnen und Schüler supports internships in enterprises and research institutions from June to September to introduce young people to activities in research in the scientific-technical field. From 2011 to 2013 a total of 4,337 funded (summer) internships for pupils were provided, during the last period 1,500 . Internships were mainly claimed by male youngsters, the percentage of participating female youngsters has however increased constantly to $38 \%$ in 2013. The youngsters mainly come from vocational colleges (Berufsbildende Höhere Schulen, BHS) - mainly secondary technical schools (HTL). Almost every third internship position was claimed by pupils from secondary academic schools 
(AHS). This can partly be explained by the fact that internships are obligatory in HTL, but not in AHS. The analysis of internships by types of organisation shows that the main group of internships are offered by enterprises, followed by universities and non-university research institutions.

The satisfaction of trainees is very high and almost constant over time. Almost all pupils rate the completed internship as very good or good. The main part of pupils regarded the requirements in the internship as (very) ambitious. More than half of the pupils indicated that their interest in a job in the field of technology and science increased after the internship. Also the institutions offering internships were very satisfied. Enterprises and research institutions gain a constant high and short-term or long-term benefit from offering internships. Internships would not have been offered without financial support in most cases.

Talente regional supports cooperative projects allowing children and young adults to deal with RTI topics for a longer period in their region. The members of the consortium shall be at least one scientific partner and two enterprise partners with reference to innovation. Furthermore, at least 5 (pre-)school institutions belonging to three out of four educational levels shall be involved. Within the first two calls 11 resp. 17 projects were supported. It was the intention of the project partners to lead the kids and youngsters to natural science and technology, to show them a variety of job possibilities and to better communicate their own research activities in the region. Teachers on the other hand found a possibility to co-operate with scientific institutions and enterprises. The exchange between pupils/small kids and researchers were the focal point, in most cases during action days where they could experiment, study, measure, analyse, etc. The last part of the project was in most cases a research event, where the kids and youngsters could present their projects to the audience.

The interviewed project partners do not only regard the sub-programme as very suitable to inspire children and youngsters for natural science and technology, but also believe that such support is absolutely necessary to increase the interest of the target group. The openness regarding topics and procedures as well as the long-term nature of the projects is regarded as strength of the sub-programme. The high practical focus and the various possibilities for interaction between researchers and children/youngsters are highly relevant.

FEMtech Forschungsprojekte focuses on applied research with gender-relevant content. In the first two calls eight projects were funded within each call. The openness considering the research topics is expressed by the rather heterogeneous project applications. The beneficiaries are mainly enterprises and research institutions that were already considering gender issues in their research activities. It is true for all projects and research institutions that the implementation would not have been possible without funding of FEMtech Forschungsprojekte due to insufficient financial means. The sub-programme contributes to considering different 
living conditions in frequently male-dominated research fields. Enterprises and research institutions are highly satisfied with the sub-programme and the persons questioned confirm interest in re-participating in FEMtech Forschungsprojekte.

FEMtech Karriere supports structural and sustainable measures that contribute to equal opportunities for women and men in RTI-enterprises and non-university research institutions in the area of nature science and technology. Each FEMtech Karriere project has two compulsory modules (gender competence and project management) and several optional modules (public relations, human resource management, work-life balance, coaching/mentoring, and career development). During the period under observation eight FEMtech Karriere projects have been funded. The beneficiaries were mainly organisations that had already experience with gender issues or organisations where the HR managers were sensitised for the topic. Although the necessity of HR development measures in general and especially measures to increase equal opportunities is recognised, resources to implement such measures are missing. Thus, funding offers the possibility for realising such measures. Effects of FEMtech Karriere projects are e.g. awareness raising regarding gender issues, especially for managers, changes in recruiting processes, increase of the number of female technicians, introduction of flexible working time models, promotion of women to management positions, etc.

The sub-programme FEMtech Praktika für Studentinnen aims at recruiting young female staff in the fields of nature science and technology. 234 (2012) and 490 (2013) internships were funded, mainly in research institutions (about $60 \%$ ) and enterprises (about $30 \%$ ). Internships could be offered from one to six months, the six months internships were preferred. Almost every fifth female student reported that the internship resulted in an employment. Feedback of the students was very good, regarding contents as well as support during the internship. The internship obviously increased the interest of the female students in natural science and technical research and in addition their specialised knowledge. The beneficiaries also gave positive feedback regarding the internships, two thirds of the participating enterprises and research institutions stated that they profited in the short or long-run from the female students. The effects of additionality were obviously very high as all beneficiaries stated that internships were only offered because of the financial funding.

In the sub-programmes FEMtech Dissertationen and Dissertationen in den Themen des BMVIT PhD-projects are funded, that are carried out by students (only women in the first call) who have to be employed by an enterprise or a nonuniversity research institution during the project. During the period under observation 5 resp. 13 projects were funded. The motivation to participate was rather the content of the project and not the aspect of supporting female postgraduates. The sub-programme helps to support women in reaching higher qualifications and motivates them to write a doctoral thesis, respectively. The female post-graduate stu- 
dents receive comprehensive assistance by the enterprises and research institutions. Especially in the research institutions the female post-graduate students seem to be well integrated in the respective research teams or the organisation as such. This, however, seems to be a challenge for enterprises in some cases. In the case of three beneficiaries the respective students were already employed full time, the others plan to employ the female post-graduate students after the end of the project or can imagine it in principle. This however means that these women managed to successfully start their work life with the help of FEMtech Dissertationen. The beneficiaries are in general very positive regarding the subprogramme.

The sub-programme Karriere-Grants supports researchers who intent to work in Austria. Travel costs for interviews (interview grants) are eligible, as well as costs for moving in case of a new employment (relocation grants) and the labour market integration of the partner (dual career grants). During the observation period 29 (2011), 130 (2012) and 90 (2013) Karriere-Grants were payed, mainly interview and relocation grants.

The Österreichische Jobbörse für Forschung, Entwicklung und Innovation is a free and open platform bringing together those offering jobs as well as those looking for a job in the field of R\&D as well as innovation. Until the end of 2013 a total of 1,031 jobs were offered, with a daily average of 333 jobs.

\section{Synthesis and conclusions}

The three intervention fields with the eight sub-programmes cover all career steps in the broadest sense and the different sub-programmes complement each other like a "funding chain". Out of the almost 1,200 organisations having benefitted from the funding portfolio, slightly more than $50 \%$ are enterprises, $17 \%$ are universities or universities of applied sciences and $8 \%$ non-university research institutions. $12 \%$ of the beneficiaries used two or more sub-programmes, and $33 \%$ received several fundings within one sub-programme. In addition the programme seems to be a "starter programme" with its partly rather low-threshold offers (especially Praktika für Schülerlnnen). $17 \%$ of the beneficiaries used with "Talente" a funding of the Austrian Research Promotion Agency for the first time. The importance of the gender issue is underlined by integrating the former programme FEMtech, but also by considering the requirements of gender criteria in other sub-programmes as well as by FEMtech Forschungsprojekte - the only sub-programme that does not aim at human resources but at gender relevant research projects. 
Possible fields for interventions can be summarised as follows:

- Praktika für Schülerinnen und Schüler: more attention should be given towards pupils having not considered studies of natural sciences or technology or a job in this area so far. Thus, information dissemination should be increased in secondary academic schools (AHS). The more intensive use of the Praktikabörse could result in a better balance regarding the educational background of the trainees.

- Talente regional: Due to the specific challenges with view to the coordination of the many project partners, an increase of the budget is suggested or the possibility to assign a higher part of the budget to the project management. The integration of the educational partners could be simplified by the reduction to two educational levels of such a project (also regarding acquisition of educational partners) and could lead to more tailormade offers. The timing for the call or the decision for funding should be determined in such a way that projects could better be considered in the plan of a new school year.

- FEMtech Forschungsprojekte: even though this refers to classical research funding the sub-programme has found its place in the Talente portfolio as one can assume that the contents have an effect on the research field and the organisations working in this field. As long as gender relevant research contents are not integrated accordingly in other programmes, such a sub-programme seems to be indispensable within the programme Talente.

- FEMtech Karriere: the programme could be adapted or split up to be able to address a broader group of addressees. FEMtech Karriere light could be a first step to implement equal opportunities. In this case only one module could be supported and the funding (and the share of own funds) could be accordingly lower. This would reduce the barriers for newcomers and SMEs. "Experienced" beneficiaries could apply for a full FEMtech Karriere project. In case of a second application it has to be guaranteed that new elements with a new focus are integrated into the project.

- FEMtech Praktika für Studentinnen: The female students already made a first step towards natural science and technology with having chosen their field of study, but they have not defined their final job, yet. Thus, factors that increase the motivation to stay in this field in this case the internship - seem to be crucial to counteract the phenomenon of the leaky pipeline (women leaving research during their career). Thus, an increase of the funds for FEMtech Praktika für Studentinnen should be considered. 
- FEMtech Dissertationen: It should be checked whether or not an increase of the funding rate for enterprises employing a postgraduate for the first time or for SMEs in general would be reasonable or necessary as they often do not have much experience in managing scientific projects. Thus, their effort to support the PhD students is higher than for research institutions.

Overall, the bundling of activities of the Austrian Ministry for Transport, Innovation and Technology (BMVIT) in the field of human resources seems to be successful and necessary to underline the importance of the topic for research as such, as there is no excellent research without excellent researchers. To increase the visibility of the programme Talente and its impact it seems to be important to establish Talente as a brand and to promote the programme and its different sub-programmes. 


\section{Einleitung}

\subsection{Gegenstand und Ziele der Evaluierung}

Die Entwicklung von Humanpotenzialen in Forschung, Technologie und Innovation (FTI) ist eine der größten Herausforderungen sowohl in Österreich als auch im europäischen Raum. Dabei gilt es, die verfügbaren Humanpotenziale quantitativ zu erweitern und ihre Qualität zu steigern. Unter der Annahme, dass die Talente grundsätzlich vorhanden sind, wurden die Aktivitäten zur Förderung der Humanpotenziale im BMVIT gebündelt und verstärkt und unter dem Förderschwerpunkt Talente subsumiert. Im Rahmen dieses Förderschwerpunkts werden die Menschen in der angewandten Forschung über den gesamten Karriereverlauf, vom Kind zum/zur etablierten Forscherln unterstützt. Übergeordnetes Ziel ist die erhöhte Ausschöpfung des Humanpotenzials im anwendungsorientierten, naturwissenschaftlich-technischen FTI-Bereich. ${ }^{1}$

Zielgruppe des Förderschwerpunkts Talente sind die (potentiellen) ForscherInnen in ihrem gesamten Karriereverlauf, d. h. Kindergartenkinder, Schulkinder, StudentInnen, DissertantInnen, NachwuchsforscherInnen bis zu etablierten Forscherlnnen, die über einreichberechtigte FörderungswerberInnen angesprochen werden. Die einreichberechtigten FörderungswerberInnen - Unternehmen, Universitäten und Fachhochschulen, außeruniversitäre Forschungseinrichtungen, Intermediäre und Einrichtungen des Technologietransfers ${ }^{2}$, jeweils mit Standort in Österreich können als erweiterte Zielgruppe verstanden werden.

Der Förderschwerpunkt Talente hat drei Interventionsfelder, in denen jeweils mehrere Programmlinien enthalten sind: ${ }^{3}$

\section{Interventionsfeld Talente entdecken: Nachwuchs}

Praktika für Schülerinnen und Schüler - Vier Wochen Technik und Naturwissenschaft

Es werden hochwertige Ferialpraktika für SchülerInnen in der technischnaturwissenschaftlichen Forschung im Zeitraum Juni bis September gefördert. Sie sollen den Jugendlichen als Bildungs- und Orientierungsangebot und als Impulsgeber für die Studien- und Berufswahl dienen. Einreichberechtigt sind Forschungseinrichtungen und forschende Unternehmen.

Vgl. Programmdokument (2011-31.12.2013) und Sonderrichtlinie (2011-30.09.2014). Talente - Der Förderschwerpunkt des BMVIT, Wien, 3.10.2011, GZ 621.120/0001-III/I2/2011

2 Bei Karriere-Grants sind die EinzelforscherInnen auch die FörderungswerberInnen.

3 Nähere Informationen zu den einzelnen Instrumenten finden sich in Kapitel 4. 
Talente regional - Kinder, Unternehmen und die Welt der Forschung

Hier werden regionale Kooperationen von (vor-)schulischen Bildungseinrichtungen und PartnerInnen aus Wirtschaft und Forschung gefördert, die junge Menschen (vom Kindergarten bis zur Matura) für Forschungs- und Innovationsthemen im Bereich Naturwissenschaft und Technik sensibilisieren und innen erste Praxiserfahrungen ermöglichen.

\section{Interventionsfeld Talente nützen: Chancengleichheit}

FEMtech Forschungsprojekte - Gendergerechte Innovation

Gefördert werden Projekte, die das Wissen für die gendergerechte Entwicklung von Produkten und Technologien umsetzen und deren Forschungsgegenstand die unterschiedlichen Lebensrealitäten und Bedürfnisse von Frauen und Männern berücksichtigt.

FEMtech Karriere - Chancengleichheit in der angewandten Forschung

Es werden strukturelle und nachhaltige Maßnahmen in Unternehmen und außeruniversitären Forschungseinrichtungen in Naturwissenschaft und Technik gefördert, durch die die Chancengleichheit von Frauen und Männern erhöht wird.

FEMtech Praktika für Studentinnen - Einstieg in die Forschungskarriere

Es werden hochwertige Praktika und Diplomarbeiten für Studentinnen in Unternehmen und außeruniversitären Forschungseinrichtungen im naturwissenschaftlich-technischen Bereich gefördert.

FEMtech Dissertationen - Unterstützung der Forschungskarriere

Gefördert werden Dissertationsprojekte aus dem naturwissenschaftlichtechnischen Bereich von Unternehmen oder außeruniversitären Forschungseinrichtungen, die eine Dissertantin aktiv unterstützen und in die Organisation integrieren.

\section{Interventionsfeld Talente finden: Forscherinnen und Forscher}

Karriere-Grants - für Vorstellungsgespräche und Umzug nach Österreich

Diese sind eine gezielte Kostenunterstützung für die Anreise nach Österreich zu einem Vorstellungsgespräch (Interview Grants), für den Umzug, wenn ein Jobwechsel nach Österreich erfolgt (Relocation Grants) oder für die Integration des Partners/der Partnerin beim Umzug nach Österreich (Dual Career Grants).

Die österreichische Jobbörse für Forschung, Entwicklung und Innovation

Die Jobbörse ist für alle frei zugänglich und kostenlos nutzbar. In der Jobbörse werden freie Stellen (von Praktika über Dissertationsstellen bis zu Senior Positions) in der österreichischen Forschung und Entwicklung bzw. Stellen mit Innovationsbezug veröffentlicht. 
Der Förderschwerpunkt Talente wurde im Jahr 2011 gestartet und wird von der Österreichischen Forschungsförderungsgesellschaft mbH (FFG) im Rahmen des Bereichs Strukturprogramme (SP) durchgeführt, wobei die Abwicklung des Förderschwerpunkts Talente in Form von Ausschreibungen erfolgt. Das BMVIT hat der FFG bis dato in zwei Ausführungsverträgen (2011 und 2012) Mittel in Höhe von rund $€ 15,350$ Mio. für die Abwicklung des Förderschwerpunkts Talente übertragen. Für die Fortsetzung des Förderschwerpunkts Talente waren im Jahr 2013 $€ 8,300$ Mio. geplant.

Laut Programmdokument ist eine Zwischenevaluierung vorgesehen, die von November 2013 bis April 2014 durchgeführt wurde. Ziel der gegenständlichen Evaluierung ist die Analyse und Reflexion des bisherigen Verlaufs des Förderschwerpunkts. Es werden die Konzeption, die Umsetzung und die bisher feststellbaren Wirkungen untersucht. Schwerpunkt der Analyse liegt auf den Interventionsfeldern Talente entdecken und Talente nützen. ${ }^{4}$ Auf Basis der Resultate werden Schlussfolgerungen und Empfehlungen für die Weiterentwicklung des Förderschwerpunkts erarbeitet. Betrachtungszeitraum sind die Jahre 2011 bis 2013, d.h. die Ausschreibungen, die in diesem Zeitraum erfolgten.

Entsprechend des strategischen Ziels des Förderschwerpunkts Talente - die Unterstützung von Menschen in der angewandten Forschung über den gesamten Karriereverlauf - setzen die Interventionsfelder und ihre Instrumente in verschiedenen Phasen der ForscherInnenlaufbahn (und sogar bereits davor) an und adressieren eine sehr heterogene Zielgruppe. Die Wirkungen der einzelnen Interventionsfelder in Hinblick auf das Globalziel - die bestmögliche Ausschöpfung des Humanpotenzials für die anwendungsorientierte, naturwissenschaftlich-technische Forschung - werden daher je nach Interventionsfeld und Zielgruppe lang-, mitteloder kurzfristig eintreten. Je früher im Karriereverlauf eine Maßnahme ansetzt, desto ungewisser ist zudem die Nachhaltigkeit der Wirkungen. Diese Überlegungen waren bei der Auswahl der Indikatoren wie auch bei der Beurteilung der Instrumente und Interventionsfelder sowie des gesamten Förderschwerpunkts zu berücksichtigen. Da es sich zudem um eine Zwischenevaluierung handelt und in einigen Programmlinien erst sehr wenige Projekte gefördert wurden, kann hier nur eine vorläufige Beurteilung des Förderschwerpunkts und der einzelnen Programmlinien erfolgen.

Der vorliegende Endbericht analysiert das Konzept des Förderschwerpunkts (Kapitel 2) und die Organisation und Umsetzung (Kapitel 3). In Kapitel 4 werden die einzelnen Programmlinien beleuchtet. Schlussfolgerungen und Empfehlungen werden in Kapitel 5 formuliert. Im Anhang finden sich Fallstudien und zusätzliche Grafiken.

4 Das Interventionsfeld Talente finden wird nur über die Analyse der Monitoringdaten untersucht. 


\subsection{Methoden}

Bei der Zwischenevaluierung des Förderschwerpunkts Talente wurde dem Ansatz der Methodentriangulation gefolgt, d.h. quantitative und qualitative Methoden wurden ergänzend angewandt. Diese sind im Folgenden kurz dargestellt:

\section{Dokumentenanalyse}

Basis der Evaluierung des Förderschwerpunkts Talente bildete eine ausführliche Analyse der vom Auftraggeber zur Verfügung gestellten Unterlagen und Dokumente (Programmdokumente, Richtlinien, Projektanträge, Projektberichte etc.). Darüber hinaus wurden Berichte der Evaluierungen der Vorgängerprogramme (brainpower austria, FEMtech,) herangezogen.

\section{Interviews mit Stakeholdern}

Insbesondere mit Blick auf die Analyse des Konzepts und des Vollzugs sowie der Organisation des Förderschwerpunkts Talente wurden folgende persönliche Interviews mit relevanten Stakeholdern des Förderschwerpunkts Talente durchgeführt:

- Gruppeninterview mit den Programmverantwortlichen im BMVIT (2 Personen)

- 3 Gruppeninterviews mit den Programmmanagerlnnen und ProgrammlinienleiterInnen der einzelnen Programmlinien in der FFG (9 Personen)

- Gruppeninterview mit der Programmleitung Talente und der Leitung Programmgruppe Humanpotenzial in der FFG (2 Personen)

\section{Analyse der FFG-Monitoringdaten}

Es erfolgte eine Analyse der von der FFG zur Verfügung gestellten Monitoringdaten zum Förderschwerpunkt Talente. Darüber hinaus beinhaltete die Datenanalyse auch die Untersuchung der Auswertungen der Feedbackfragebögen der FördernehmerInnen und PraktikantInnen in den Programmlinien Praktika für Schülerinnen und Schüler und FEMtech Praktika für Studentinnen. Diese enthalten neben quantitativen Analysen auch qualitative Aussagen der Befragten auf offene Fragen.

\section{Onlinebefragung}

Im Zuge der Onlinebefragung wurden 184 PädagogInnen bzw. (vor-)schulische Bildungseinrichtungen, die in ein Talente regional Projekt (erste und zweite Ausschreibungsrunde) involviert waren/sind, zur Teilnahme eingeladen. Insgesamt konnten 53 ausgefüllte Fragebögen für die Auswertung berücksichtigt werden (dies entspricht einer Rücklaufquote von $29 \%$ ).

Der überwiegende Anteil der Antwortenden sind dem Sekundären Bildungsbereich zuzuordnen (68 \%) - vorwiegend AHS und BHS, während $15 \%$ der Befragten in Volksschulen tätig sind bzw. weitere $17 \%$ in Kindergärten. 
Mehr als drei Viertel der Antwortenden (79\%) sind in den Bildungseinrichtungen als KindergartenpädagogInnen oder LehrerInnen tätig, weitere $21 \%$ in der Doppelfunktion Lehrerln-Direktorln.

\section{Fallstudien}

Es wurden insgesamt 10 Fallstudien von Projekten im Rahmen von Talente regional (4), FEMtech Karriere (3) und FEMtech Forschungsprojekte (3) durchgeführt. Die Auswahl der Fallstudien erfolgte auf der Basis der Analyse der Projektanträge und Projektberichte. Der Fokus wurde auf Projekte gelegt, die mit Ende 2013 (fast) abgeschlossen waren. D.h. dass bei Talente regional nur Projekte aus der ersten Ausschreibungsrunde berücksichtigt wurden ebenso wie bei FEMtech Forschungsprojekte.

Neben der detaillierten Dokumentenanalyse wurden im Rahmen der Fallstudien bei Talente regional Interviews mit der Projektleitung und den wissenschaftlichen PartnerInnen, bei FEMtech Karriere Interviews mit Projektverantwortlichen und teilweise der Geschäftsführung und bei FEMtech Forschungsprojekte mit den ProjektleiterInnen durchgeführt.

\section{Interviews mit Projektverantwortlichen von FEMtech Dissertationen}

Für die Analyse der Programmlinie FEMtech Dissertationen wurden telefonische Interviews mit den ProjektnehmerInnen durchgeführt. Da sich die Projekte der zweiten Ausschreibung zum Zeitpunkt der Evaluierung erst in der Startphase befanden, konnten dabei nur die fünf im Zuge der ersten Ausschreibung geförderten Unternehmen und Forschungseinrichtungen in die Evaluierung einbezogen und entsprechend befragt werden. 


\section{Das Konzept des Förderschwerpunkts Talente}

Der Förderschwerpunkt Talente bündelt die Förderungen des BMVIT im Bereich Humanpotenzial. Damit wurden 2011 die bis dahin existierenden Programme (brainpower austria, FEMtech, Forschung macht Schule bzw. generation innovation), die auf die Förderung des Humanpotenzials in der Naturwissenschaft und Technik abzielten, unter ein Dach zusammengefasst. Der Förderschwerpunkt fokussiert auf den gesamten Karriereverlauf von Forscherlnnen im weitesten Sinn, d. h. es werden (potentielle) Forscherlnnen im Kindesalter ebenso gefördert wie etablierte ForscherInnen. Der Förderschwerpunkt gliedert sich in drei Interventionsfelder mit insgesamt acht Programmlinien:

- Talente entdecken: Nachwuchs

○ Praktika für Schülerinnen und Schüler

- Talente regional

- Talente nützen: Chancengleichheit

○ FEMtech Forschungsprojekte

- FEMtech Karriere

- FEMtech Praktika für Studentinnen

- FEMtech Dissertationen

- Talente finden: Forscherinnen und Forscher

- Karriere-Grants

- Die österreichische Jobbörse für Forschung, Entwicklung und Innovation

Die folgende Grafik (siehe Grafik 1) verortet die Programmlinien entlang des „Entwicklungspfads“ (potenzieller) Forscherlnnen bzw. entlang einer „Forscherlnnenkarriere". Sie illustriert, dass der Förderschwerpunkt alle Karrierestationen im weitesten Sinn abdeckt, und dass die verschiedenen Programmlinien ineinandergreifen. Zudem ist ersichtlich, dass jede „Karrierestation“ (mit Ausnahme der Vorschulkinder) von mindestens zwei Programmlinien adressiert wird und somit (potentielle) ForscherInnen in jedem Stadium ihrer Karriere von mindestens zwei unterschiedlichen Fördermöglichkeiten profitieren können. Als Querschnittsmaterie wird "Gender" zudem in fast allen Programmlinien explizit berücksichtigt. 
Grafik 1 Die Programmlinien des Forschungsschwerpunkts Talente
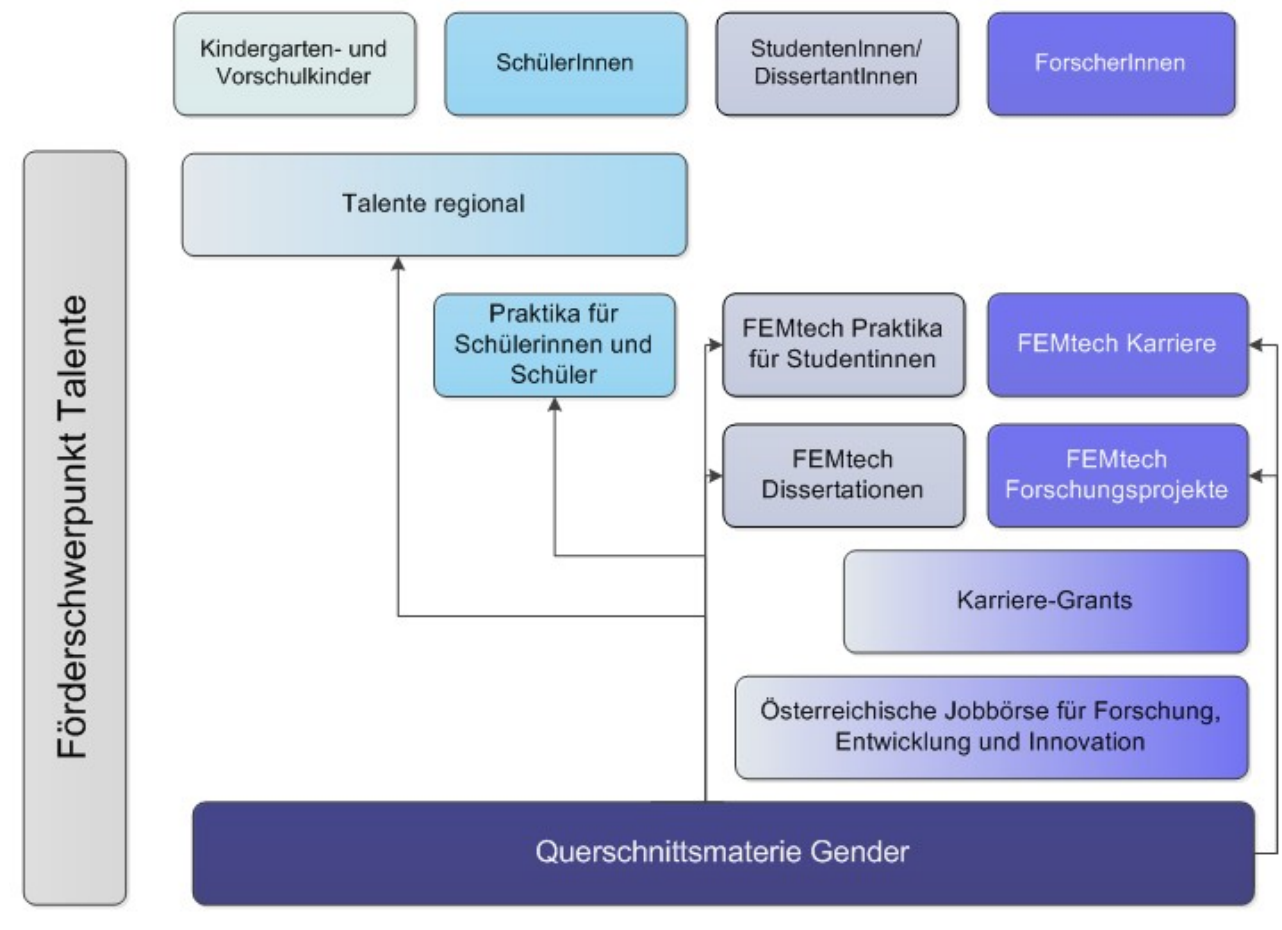

Quelle: KMU Forschung Austria

Somit entspricht das Konzept von Talente seinem Ziel, Menschen in der angewandten Forschung über den gesamten Karriereverlauf zu unterstützen, um das Humanpotenzial für den anwendungsorientierten, naturwissenschaftlich-technischen FTI-Bereich besser ausschöpfen zu können. Der Förderschwerpunkt setzt beim jüngsten Nachwuchs mit bewusstseinsbildenden Maßnahmen an (Praktika für Schülerlnnen, Talente regional) und zielt auf die Verbesserung von Rahmenbedingungen durch die Förderung der Vernetzung von (vor-)schulischen Bildungseinrichtungen mit Partnern aus Wissenschaft und Wirtschaft, die wiederum motiviert werden, langfristige Nachwuchspflege auf ihre Agenda zu setzen (Talente regional).

Um das Potential von Frauen zu fördern und deren Unterrepräsentanz in Naturwissenschaft und Technik bzw. im FTI-Bereich zu bekämpfen, werden einerseits Maßnahmen gesetzt, um Studentinnen und Absolventinnen zu einem Berufseinstieg und einer Höherqualifizierung in einem naturwissenschaftlich-technischen Bereich zu motivieren (FEMtech Praktika für Studentinnen, FEMtech Dissertationen), andererseits werden Unternehmen gefördert, die Chancengleichheit in ihrem Unternehmen verankern wollen (FEMtech Karriere). Zur Unterstreichung der Relevanz des Genderthemas werden zudem Forschungsprojekte mit genderrelevantem Inhalt gefördert (FEMtech Forschungsprojekte) und finden sich in einigen Bereichen zusätzliche Vorgaben zur Integration von Genderkriterien (Talente regional, Praktika für Schülerlnnen). 
Maßnahmen zur Vernetzung von ForscherInnen aus dem In- und Ausland mit Unternehmen und Forschungseinrichtungen in Österreich (Jobbörse) und zur Unterstützung von Forscherlnnen, die ihre Karriere in Österreich fortsetzen wollen (Karriere-Grants), runden das Angebot ab.

Der Förderschwerpunkt zeichnet sich somit durch eine hohe Flexibilität und eine große Auswahl an Instrumenten aus, mit denen unterschiedlichste Zielgruppen erreicht werden können und die auch wichtige Schnittstellen und kritische Phasen im Karriereverlauf adressieren. Es werden Kleinkindern mit ersten bewusstseinsbildenden Maßnahmen adressiert, Schülerlnnen, die vor Ausbildungsentscheidungen stehen (Wahl von Schulzweigen, Wahl von Lehrberufen, Studienwahl), Studentinnen, die vor einer Berufsentscheidung stehen, Technikerinnen und Forscherinnen, die vor Karriereentscheidungen stehen, sowie ForscherInnen, die sich in Österreich etablieren wollen. Zudem setzen alle Programmlinien mit Ausnahme von Karriere-Grants nicht nur auf die Förderung des Individuums, sondern auch auf die Verbesserung von Rahmenbedingungen, indem Unternehmen und Forschungseinrichtungen als FördernehmerInnen für die Förderung von Humanpotenzial sensibilisiert werden. Es gibt in Österreich kein anderes Förderprogramm im Bereich FTI und Humanressourcen, das eine so breit definierte Zielgruppe adressiert.

In der FFG hat der Förderschwerpunkt insofern eine Sonderstellung als es sich bei den Programmlinien (mit Ausnahme von FEMtech Forschungsprojekte und teilweise FEMtech Dissertationen) um keine klassische Forschungsförderung handelt, sondern der "Mensch“ in den Mittelpunkt gerückt wird. Auch handelt es sich bei mehreren Programmlinien um ein sehr niederschwelliges Angebot, sowohl in Hinblick auf administrative Anforderungen bei der Einreichung als auch bezüglich der Förderhöhe (z B. Praktika für SchülerInnen, FEMtech Praktika für Studentinnen), die damit eine beträchtliche Breitenwirkung haben. Über die Förderung des Humanpotenzials hinaus hat der Förderschwerpunkt Talente damit zudem einen Mehrwert für die Förderagentur, da insgesamt das Interesse an der Forschung auch bei weniger forschungsaffinen Gruppen geweckt und erhöht werden kann.

Diese Besonderheiten bringen jedoch auch einige Nachteile mit sich. Da es sich eben bei den meisten Programmlinien nicht um Forschungsförderung im klassischen Sinn handelt, fehlt es dem Thema Humanressourcen oftmals am nötigen Backup innerhalb der Community. Dadurch weisen einzelne Programmlinien unterkritische Größen auf. Insgesamt scheint auch nicht klar, inwieweit die Themen Humanressourcen und Bildung Schwerpunkte des BMVIT sind bzw. sein können. Diese etwas unklare Stellung des Förderschwerpunkts ist vielleicht auch vor dem Hintergrund zu sehen, dass die Marke Talente noch nicht etabliert und ausreichend bekannt ist. 
Die Integration der Vorgängerprogramme in den Förderschwerpunkt kann als durchaus gelungen betrachtet werden. Durch die Bündelung aller Förderungen im Bereich des Humanpotenzials ist es gelungen, eine Förderkette zu etablieren und somit die Förderungen über den gesamten Karriereverlauf der ForscherInnen erkennbar und zuordenbar zu machen. Allerdings sind diese Zusammenführung und das gemeinsame Dach noch nicht ausreichend bekannt, im Vergleich zu den Vorgängerprogrammen. Um den Förderschwerpunkt nachhaltig zu positionieren, scheint daher ein Branding von Talente notwendig, ebenso wie weitere Bemühungen, damit die einzelnen Programmlinien unter dem Förderschwerpunkt vollständig aufgehen können. 


\section{Organisation und Umsetzung}

Mit dem Förderschwerpunkt Talente und seinem Fokus auf Humanressourcen hat die FFG Aufgaben übernommen, die nicht in ihr Kerngeschäft - die Forschungsförderung im klassischen Sinn - fallen. Auch handelt es sich bei einigen Programmlinien um Kleinstförderungen (Praktika, Karriere-Grants), was zusätzlich zur Sonderstellung des Förderschwerpunkts innerhalb der FFG beiträgt. Die MitarbeiterInnen zeigen sich aber nicht nur von der Wichtigkeit dieser Fördermaßnahmen überzeugt, das „Besondere“ am Förderschwerpunkt führt auch zu einem sehr ausgeprägten Engagement seitens der FFG-MitarbeiterInnen.

Dieses „Besondere“ spiegelt sich auch bei den Fördernehmerlnnen und involvierten Personen wider, die teilweise nicht der klassischen FFG-Klientel entsprechen und die niederschwelligen Angebote nützen. Einzelfoscherlnnen (bei KarriereGrants) und Erstfördernehmerlnnen ${ }^{5}$ (v. a. bei Praktika für Schülerlnnen) sind im Förderschwerpunkt Talente vertreten, aber selbst bei Organisationen, die schon Förderungen der FFG in Anspruch genommen haben, sind es andere Ansprechpersonen, wie etwa die HR-Abteilungen (z. B. bei FEMtech Karriere), als üblicherweise. Diese Förderneulinge benötigen intensivere Betreuung, sowohl in der Phase der Antragsstellung als auch im Zuge der Abwicklung. Auch kommt es zu speziellen Fragen in Zusammenhang mit der Förderung von Organisationen und Personen (z. B. Bildungseinrichtungen und LehrerInnen bei Talente regional), die in Projekte integriert und in deren Rahmen gefördert werden, aber der FFG grundsätzlich eher fern stehen. Hier sind Beratungsgespräche gefragt, die bei den AntragstellerInnen wenig Vorwissen voraussetzen können. Die sehr breit gefasste Zielgruppe des Förderschwerpunkts Talente sowie die kleinteilige Struktur mit den zahlreichen unterschiedlichen Programmlinien erfordern damit eine vergleichsweise hohe Betreuungsintensität, der die FFG durch ein relativ großes Betreuungsteam begegnet. Darüber hinaus trägt das sehr ausführliche Monitoringsystem (z. B. Feedback-Fragebögen der PraktikantInnen) zur Ressourcenintensität des Förderschwerpunktes bei.

Durch die Bündelung der verschiedenen Aktivitäten zur Förderung des Humanpotenzials in den Förderschwerpunkt Talente stand die FFG vor der Aufgabe, diese auch organisatorisch zusammenzuführen. Dies gestaltete sich in den Fällen etwas aufwendiger, in denen zuvor die Förderung von einer anderen Stelle als der FFG abgewickelt wurde. V. a. im Bereich generation innovation hat es bereits zwei Ausschreibung für die neue Programmlinie Talente regional gegeben. In diesem Fall

$517 \%$ der Fördernehmerlnnen haben im Rahmen von Talente das erste Mal eine Förderung bei der FFG in Anspruch genommen (v. a. in der Programmlinie Praktika für Schülerlnnen), wenn man die Karriere-Grants hinzuzählt, die allerdings auf eine einmalige Förderung ausgelegt sind, sind es sogar 33 \% (Quelle: FFG- Monitoringdaten) 
mussten die Verträge FFG-kompatibel umgestaltet werden. In anderen Fällen, in denen die FFG schon zuvor die Förderungen abgewickelt hatte, verlief der Übergang unproblematisch. Grundsätzlich dürfte die Zusammenführung aus organisatorischer Sicht gelungen sein.

Das gemeinsame Dach unterstützt einen besseren Austausch (es finden regelmäBige Austauschtermine innerhalb der FFG statt) und eine bessere Vernetzung der FFG-Programmverantwortlichen. Damit können die Kundlnnen in der Beratung auch auf die anderen Programmlinien verwiesen werden, was der Idee der Förderkette entspricht.

Dadurch, dass die FFG nur mehr zwei Ansprechpartnerlnnen im Ministerium hat, die für alle Programmlinien zuständig sind, hat sich auch die Abstimmung wesentlich erleichtert und die Rollenaufteilung und Verantwortlichkeiten sowie die Entscheidungsstrukturen sind klar und strukturiert. Vor der Integration war mit dem FEMtech Kompetenzzentrum und der Koordinationsstelle von generation innovation weitere Akteurlnnen in die Programmabwicklung involviert, was laut FFG teilweise zu unklaren Kompetenzaufteilungen und schwierigen Entscheidungsfindungsprozessen geführt hat.

Diese AkteurInnen verliehen den Programmlinien jedoch im Gegenzug erhöhte Sichtbarkeit und setzten verstärkt Begleitmaßnahmen. Die Koordinationsstelle hatte beispielsweise die (personellen) Ressourcen, systematisch generation innovation im Bildungsbereich bekannt zu machen. Über das Kompetenzzentrum konnte die Marke FEMtech im Laufe der Zeit etabliert werden und ist noch immer ein Begriff. Dagegen ist noch wenig bekannt, dass FEMtech nun Teil des Förderschwerpunkts Talente ist. Daher scheinen hier zum einen ein Branding von Talente notwendig, zum anderen aber auch weitere Anstrengungen zur vollständigen Integration der einzelnen Programmlinien unter den Förderschwerpunkt und seine Zielsetzungen.

\section{Ausschreibung und Auswahlverfahren}

Alle Programmlinien mit Ausnahme von FEMtech Karriere und Karriere-Grants (laufende Einreichung möglich) haben vorgegebene Ausschreibungszeiträume, in denen Einreichungen möglich sind. Bei den Auswahlverfahren kommen zwei FFGModelle zur Anwendung:

- Modell 1 steht für verkürzte Antragsverfahren mit einem vereinfachten Bewertungsschema. Die Förderungsentscheidung trifft die Geschäftsführung der FFG im Auftrag des BMVIT. Dieses Modell wird für Praktika für SchülerInnen und Schüler, FEMtech Praktika für Studentinnen und KarriereGrants angewandt, sowie bei FEMtech Karriere und FEMtech Dissertationen unter Einholung von Fachgutachten. 
- Modell 3 als Wettbewerbsverfahren, bei dem die Bewertung durch FFGinterne und externe Fachgutachten erfolgt auf deren Basis ein standing committee eine Förderungsempfehlung abgibt. Das BMVIT trifft die Förderungsentscheidung. Dieses Verfahren kommt bei Talente regional und FEMtech Forschungsprojekte zur Anwendung.

Grundsätzlich werden Ausschreibungsart und Auswahlverfahren sowohl von der FFG als auch von den im Rahmen der Fallstudien befragten Fördernehmerlnnen als adäquat für die jeweilige Programmlinie gesehen und es gibt nur einzelne Kritikpunkte. Bei Talente regional gibt es beispielsweise die Anregung, die Ausschreibungszeitpunkte bzw. Förderungsentscheidungen besser auf das Schuljahr abzustimmen. Da die Jurysitzungen bis dato im März stattfinden, fällt der Förderentscheid und in der Folge der Startschuss für die Projekte oft erst am Ende des Schuljahres. Zu diesem Zeitpunkt ist aber das im Herbst beginnende Schuljahr oft schon sehr verplant, was die Koordinierung der Aktivitäten im Projekt deutlich erschwert (siehe Kapitel 4.1.2.). Hier wird eine Verschiebung der Ausschreibung vorgeschlagen bzw. könnte eine raschere Förderentscheidung den besonderen Rahmenbedingungen dieser Projekte entgegenkommen.

Auch bei FEMtech Dissertationen erscheint der Zeitpunkt zwischen Ausschreibungsbeginn und Förderungsentscheid eher lang, was beispielsweise dazu geführt hat, dass in der ersten Ausschreibungsrunde eine Dissertantin abgesprungen ist. Kürzere Ausschreibungszeiträume und raschere Entscheidungen könnten hier eine höhere Planbarkeit unterstützen.

Kontroversiell werden in der FFG auch die themenbezogenen Ausschreibungen gesehen. Diese führen zwar einerseits zu einer Fokussierung, andererseits erschwert ein jährlicher Themenwechsel die Mobilisierung der Zielgruppe, da immer wieder neue potenzielle Fördernehmerlnnen angesprochen werden müssen. Eine offene Ausschreibung mit Themenschwerpunkt könnte hier Abhilfe schaffen.

\section{Beurteilung der FFG durch die FördernehmerInnen}

Die FördernehmerInnen zeigen sich in allen näher untersuchten Programmlinien grundsätzlich sehr zufrieden mit der Betreuung durch die FFG.

Die Zufriedenheit der Fördernehmerlnnen mit der Instrumentenabwicklung in der Programmlinie Praktika für Schülerinnen und Schüler drückt sich in konstant positiven Beurteilungen aus. Sowohl die persönliche Beratung und das angebotene Informationsmaterial, als auch die Abwicklung der Praktikabörse werden von den Fördernehmerlnnen mit Durchschnittsnoten von 1,2 bis 1,4 (2013) beurteilt. ${ }^{6}$ Gleiches lässt sich für die Programmlinie FEMtech Praktika für Studentinnen feststel-

6 Quelle: Talente entdecken: Schülerinnen und Schüler 2013, Feedback FörderungsnehmerInnen 
len. Die Zufriedenheit der FördernehmerInnen pendelt in den beiden Ausschreibungsjahren zwischen durchschnittlichen Bewertungen von 1,2 bis $1,5{ }^{7}$

Im Rahmen von Talente regional sprechen die für die Fallstudien Befragten von einer sehr guten Betreuung durch die FFG. Auch die vorbereiteten Hilfstools (Infoblätter, Antragsformular, Checkliste etc.) für die Kooperationszuschüsse werden als sehr hilfreich und zweckmäßig bezeichnet. Nur die Vorlage für den Konsortialvertrag wird von einer Befragten als nicht geeignet für solche Projekte empfunden.

Auch die Verantwortlichen der untersuchten FEMtech Karriere Projekte betonen, dass sie sich sehr gut und intensiv betreut gefühlt haben, und dass diese Betreuung zum Erfolg der Projekte beigetragen habe. Auch wird positiv hervorgehoben, dass in den Ausschreibungsunterlagen die Module näher erläutert und auch anhand von Beispielen konkrete Maßnahmen illustriert werden. Diese Beispiele werden als sehr hilfreich eingestuft.

Ähnlich positives Feedback gibt es von den Interviewten im Rahmen von FEMtech Dissertationen, die auch die FFG-MitarbeiterInnen als sehr kompetent und hilfsbereit beschrieben. Anfragen würden rasch und unbürokratisch behandelt.

„Antragstellung war gut, sehr viel direkter Kontakt mit der FFG, das war sehr hilfreich, viel Hilfestellung durch die FFG. Template von FFG ist sehr klar, selbsterklärend."

Die befragten Fördernehmerlnnen von FEMtech Forschungsprojekte zeigten sich in der Projektdurchführung ebenso zufrieden mit dem Angebot, der Betreuung und der Abwicklung der Fördermaßnahme durch die FFG. Im Speziellen beurteilten die Fördernehmerlnnen den projektbezogenen administrativen Aufwand und die Anforderungen seitens der FFG als überschaubar und transparent gestaltet. In Bezug auf die Förderausschreibung wurde seitens einer Fördernehmerln allerdings die starke Produkt- bzw. Technologieorientierung kritisiert. So müssen im Antrag schon Outputs beschrieben werden, welche erst am Ende des F\&E-Prozesses stehen, und im Vorfeld nur schwer abgeschätzt werden können. Hier wären inhaltliche Hilfestellungen seitens der FFG bezüglich relevanter Outputs der Projektdurchführung hilfreich.

7 Quelle: Feedback der FörderungsnehmerInnen FEMtech Praktika für Studentinnen 


\section{Die einzelnen Programmlinien}

\subsection{Talente entdecken: Nachwuchs}

\subsubsection{Praktika für SchülerInnen}

Die Programmlinie Praktika für Schülerlnnen soll durch die Förderung von SchülerInnenpraktika in Unternehmen und Forschungseinrichtungen Jugendlichen die Tätigkeiten in der Forschung im naturwissenschaftlich-technischen Bereich näherbringen und sie für diese begeistern.

Tabelle 1 Eckpunkte von Praktika für Schülerlnnen

\begin{tabular}{|c|c|}
\hline Praktika für SchülerInnen & Kurzbeschreibung \\
\hline Ziele & $\begin{array}{l}\text { Junge Menschen sollen für Forschung und Entwicklung be- } \\
\text { geistert werden. }\end{array}$ \\
\hline Zielgruppen & $\begin{array}{l}\text { Unternehmen, Universitäten, Fachhochschulen, Kompetenz- } \\
\text { zentren, Forschungseinrichtungen (Fördernehmerlnnen). } \\
\text { Schülerlnnen (österreichische Schule), die mindestens } 15 \\
\text { Jahre alt sind. In allen Anträgen mit drei oder mehr Praktika } \\
\text { muss zumindest ein Drittel der Praktikumsplätze an Mädchen } \\
\text { vergeben werden. }\end{array}$ \\
\hline Inhalt & $\begin{array}{l}\text { Praktika für SchülerInnen im Zeitraum Juni bis September im } \\
\text { FTI-Bereich mit technischem oder naturwissenschaftlichem } \\
\text { Bezug }\end{array}$ \\
\hline Einreichverfahren & $\begin{array}{l}\text { Laufende Einreichung nach Ausschreibung (Jänner bis Juli } \\
2011,2012, \quad 2013 \text { ), bei Mittelausschöpfung vorzeitiges } \\
\text { Schließen der Ausschreibung, } 2013 \text { Schwerpunkt Mobilität }\end{array}$ \\
\hline Laufzeit & $\begin{array}{l}\text { Praktikumsdauer mindestens } 4 \text { Wochen } \\
\text { (mind. } 28,5 \text { Wochenstunden) }\end{array}$ \\
\hline Förderhöhe & $€ 1.000$,- pro Praktikum (davon $€ 700$,- Praktikumsentgelt) \\
\hline
\end{tabular}

Im Zeitraum 2011 bis 2013 wurden in Summe 4.337 geförderte (Ferial-)Praktikumsplätze für Schülerlnnen im Bereich von technischen bzw. naturwissenschaftlichen FTI-Aktivitäten zur Verfügung gestellt, im Zuge der letzten Ausschreibung waren es über 1.500. Die Möglichkeit, Einblicke in die Tätigkeitsfelder von Forscherlnnen zu gewinnen, wurde zum überwiegenden Teil von männlichen Interessenten in Anspruch genommen, jedoch konnte von Jahr zu Jahr der Anteil weiblicher PraktikantInnen angehoben werden. So stieg der Anteil der teilnehmenden Schülerinnen von 32 \% im Jahr 2011 auf 38 \% im Jahr 2013 (siehe Grafik 2). Die Zielsetzung der Programmlinie, zumindest ein Drittel der Praktikumsplätze an Schülerinnen zu vergeben, konnte damit erreicht und zuletzt sogar übertroffen werden. 
Grafik 2 Anteil weiblicher bzw. männlicher Praktikumsteilnehmerlnnen

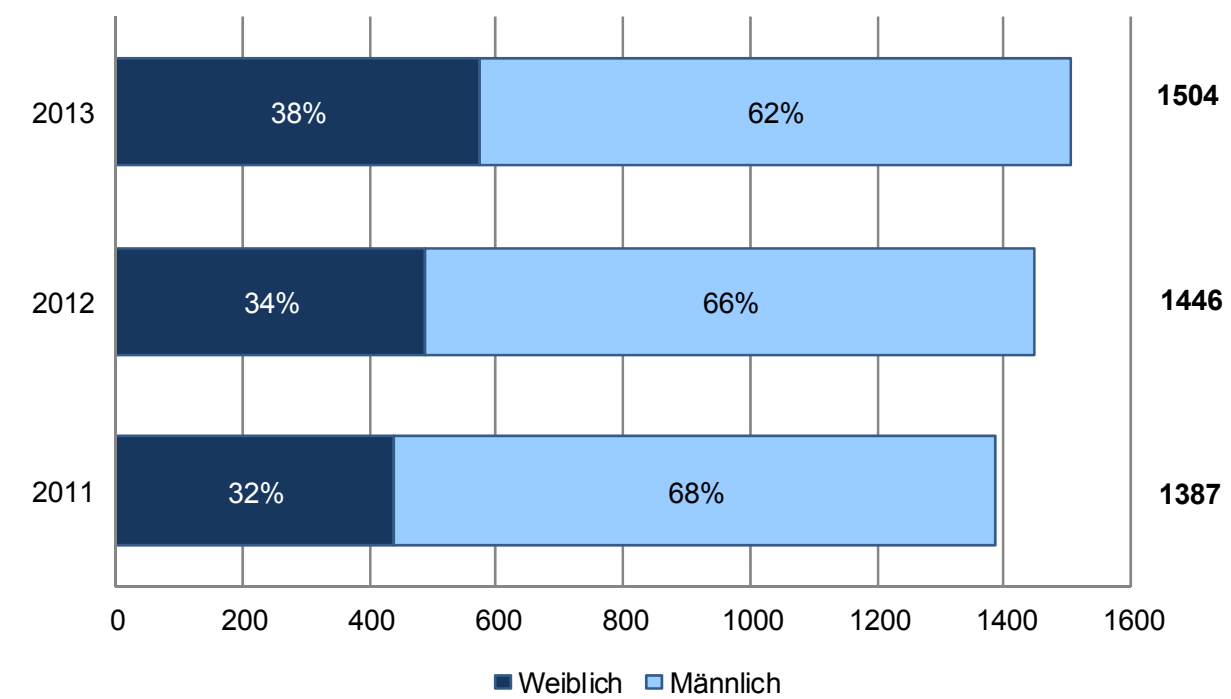

Quelle: FFG-Monitoring-Daten

Die PraktikantInnen rekrutieren sich zum überwiegenden Teil aus Berufsbildenden Höheren Schulen (BHS) - vorwiegend HTL. Rd. zwei Drittel der SchülerInnen (2013: $69 \%$ ), die erste Berufserfahrungen im technisch-naturwissenschaftlichen Forschungsbereich sammelten, waren diesem Schultypus zuzurechnen. Knapp jeder dritte Praktikumsplatz (2013: $28 \%$ ) wurde von AHS-SchülerInnen besetzt, während die übrigen den sonstigen Schultypen entstammten (Fachschulen, Bundes-Oberstufenrealgymnasium etc.). Der Anteil der PraktikantInnen aus BHS hat sich darüber hinaus im Zeitablauf leicht erhöht (siehe Grafik 12 im Anhang). Der angestrebte Zielwert von $50 \%$ PraktikantInnen aus AHS und sonstigen Schulen konnte damit nicht erreicht werden. ${ }^{8}$ Dies ist wohl u. a. darauf zurückzuführen, dass in den HTLs verpflichtende Ferialpraktika vorgesehen sind, während dies bei AHS-SchülerInnen nicht der Fall ist.

Der hohe Anteil an SchülerInnen aus BHS erklärt auch, warum Mädchen nur zu etwa einem Drittel die Praktika in Anspruch nehmen. Während aus den AHS rd. die Hälfte der PraktikantInnen Mädchen sind, ist es bei den BHS nur ein Drittel. Hier spielt natürlich der geringe Mädchenanteil in HTLs eine entscheidende Rolle. Die Altersverteilung der PraktikantInnen zeigt über alle drei Untersuchungsjahre hinweg, dass die 17- bis 18-jährigen (11. bis 12. Schulstufe) am häufigsten Praktika in Anspruch genommen haben (jeweils rund $55 \%$ ).

8 Programmdokument und Sonderrichtlinien Talente - Der Förderschwerpunkt des BMVIT 
Die Verteilung der Praktikumsplätze nach Organisationsart zeigt, dass Unternehmen die deutlich größte Gruppe der Praktikumsgeberlnnen (2013: $60 \%$ insgesamt, davon: $34 \%$ Großunternehmen, $21 \%$ mittlere Unternehmen und $6 \%$ Kleinunternehmen) bilden, gefolgt von den Universitäten (2013: $18 \%$ ) und den außeruniversitären Forschungseinrichtungen (2013: $10 \%$ ). Die verbliebenen rd. $12 \%$ der durchgeführten Praktika teilen sich auf Fachhochschulen, Kompetenzzentren und sonstige Organisationen auf. Diese Anteile änderten sich im Betrachtungszeitraum kaum. Wirft man einen Blick auf die Geschlechterverteilung nach Organisationsart, so zeigen sich deutliche Unterschiede zwischen Unternehmen und Forschungseinrichtungen. Während die SchülerInnen in universitären und außeruniversitären Einrichtungen 2013 zwischen $43 \%$ und $45 \%$ der Praktikumsstellen besetzt haben, waren es bei den Unternehmen nur $27 \%$ (KU) bis $37 \%(\mathrm{GU})$ und bei den Kompetenzzentren $36 \%$. Zwar hat sich der Anteil weiblicher Praktikantinnen in Unternehmen gegenüber 2012 leicht verbessert, allerdings liegt der Anteil bei den KMU immer noch um 12 bis 16 Prozentpunkte hinter den Forschungseinrichtungen.

Knapp zwei Drittel der Praktikumstätigkeiten waren im Bereich der technischen Wissenschaften, ein weiteres Drittel im naturwissenschaftlichen Bereich angesiedelt. Die Bereiche Humanmedizin, Land- und Forstwirtschaft sowie Veterinärmedizin waren nur bei rd. $4 \%$ der Praktikumsstellen vertreten. Im Themenfeld der technischen Wissenschaften wurde im Jahr 2013 nur jede dritte Praktikumstelle weiblich besetzt, während dieser Anteil in den Naturwissenschaften bei $44 \%$ lag. Ein ausgeglichenes Geschlechterverhältnis lies sich nur im Forschungsbereich Humanmedizin feststellen. Die regionale Verteilung im Zeitverlauf zeigt, dass die Steirischen und Wiener Forschungseinrichtungen und Unternehmen im Durchschnitt jeweils rd. ein Fünftel der Praktikumsplätze zur Verfügung gestellt haben. Ebenfalls im zweistelligen Prozentbereich lagen die Praktikumsplätze der Länder Oberösterreich, Niederösterreich und Kärnten, während die Unternehmen und F\&EEinrichtungen der übrigen Bundesländer im einstelligen Prozentbereichen partizipiert haben.

Der größte Teil der SchülerInnen hat über das persönliche Umfeld von der Möglichkeit eines geförderten Praktikums erfahren (2013: 80 \%). Darüber hinaus sind die ArbeitgeberInnen selber (50\%) bzw. die Schulen (42\%) aktiv in der Bewerbung von Praktika. Mädchen bedienen sich, um an ein Praktikum zu gelangen, eher der Praktikumsbörse. Allerdings wird nur ein sehr kleiner Teil der Praktikumsplätze über diese Börse (2012: 16 \%, 2013: 15 \%) angeboten.

Eine Beurteilung des Praktikums durch die Schülerlnnen erfolgt durch eine direkte Befragung nach Praktikumsende (siehe Grafik 3). Die Zufriedenheit mit dem Praktikum liegt demzufolge auf sehr hohem Niveau und ist im Zeitverlauf nahezu konstant. 
Mehr als $95 \%$ der Schülerlnnen bewerten das absolvierte Praktikum mit sehr gut oder gut (mehr als zwei Drittel „sehr gut"). Die Beurteilung mit „sehr gut“ ist im Zeitablauf gesunken.

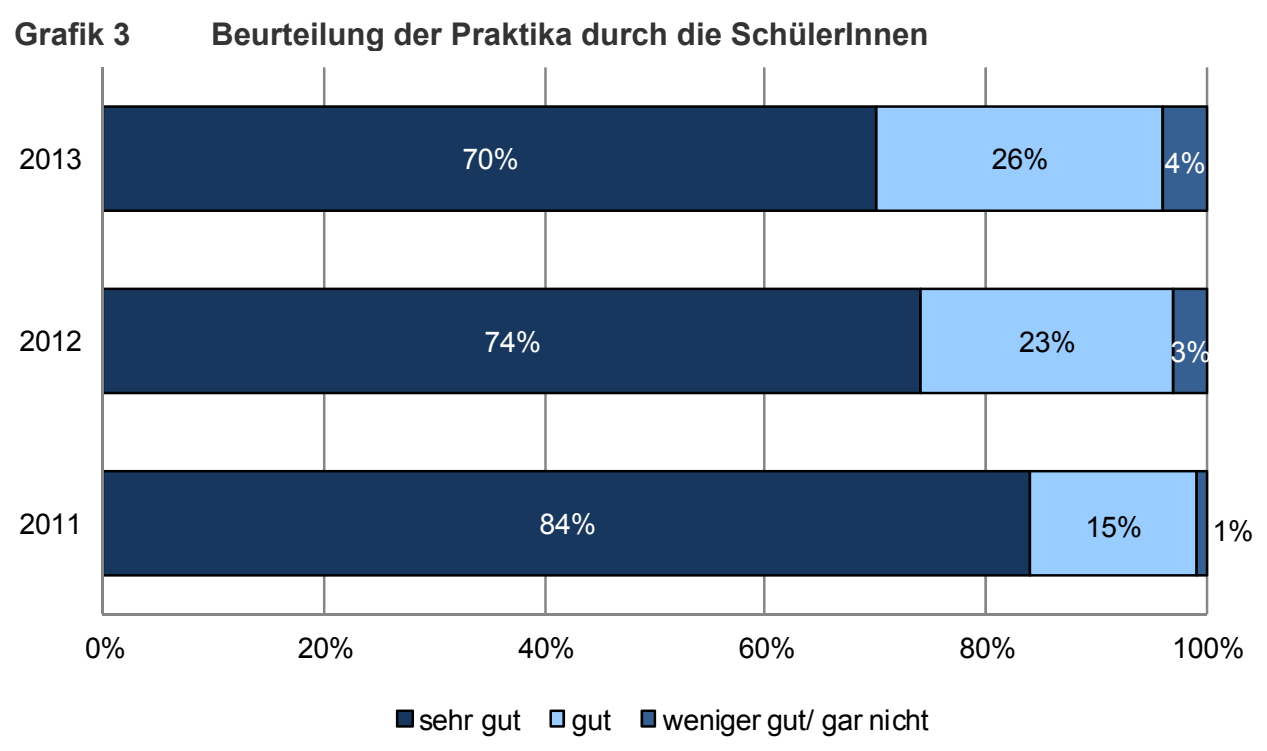

Quelle: Talente entdecken: Praktika für Schülerinnen und Schüler 2013, Feedback Schülerlnnen; $\mathrm{n}=133$ (2011), 164 (2012), 187 (2013)

Stellvertretende Kommentare der SchülerInnen unterstreichen die hohe Zufriedenheit.

„ICh habe gute Einblicke in die Welt der Forschung bekommen und konnte mich mit interessanten Themen auseinandersetzen."

„ICh habe erste Eindrücke in der Arbeitswelt gesammelt, konnte Einblicke in die Forschung tätigen und habe zusätzlich etwas Geld verdient."

Die Praktika dürften inhaltlich ein sehr hohes Niveau aufweisen (siehe Abbildung 13 im Anhang). Der überwiegende Teil der Schülerlnnen (mehr als $80 \%$ ) bewertete die inhaltlichen Anforderungen als (sehr) anspruchsvoll. Im Zeitverlauf ist der Anteil der inhaltlich als (sehr) anspruchsvollen beurteilten Praktika allerdings leicht gesunken (83\% im Jahr 2013 gegenüber $92 \%$ im Jahr 2011). Damit dürften die Beurteilung der Praktika insgesamt und die Beurteilung des Anspruchsniveaus korrelieren.

Mehr als die Hälfte der befragten SchülerInnen gab an, dass ihr Interesse an einem Beruf im technisch-naturwissenschaftlichen Bereich auf Grund des Praktikums gestiegen ist (siehe Grafik 4). Auch hier ist ein leicht abnehmender Trend bemerkbar (von 73 \% 2011 auf 57 \% 2013). Negative Wirkungen auf das Interesse, einen solchen Beruf auszuüben, hat das Praktikum allerdings in den wenigsten Fällen ( $1 \%$ bis $3 \%)$. 


\section{Grafik 4 Veränderung des Interesses der PraktikantInnen an technisch-} naturwissenschaftlichen Berufen

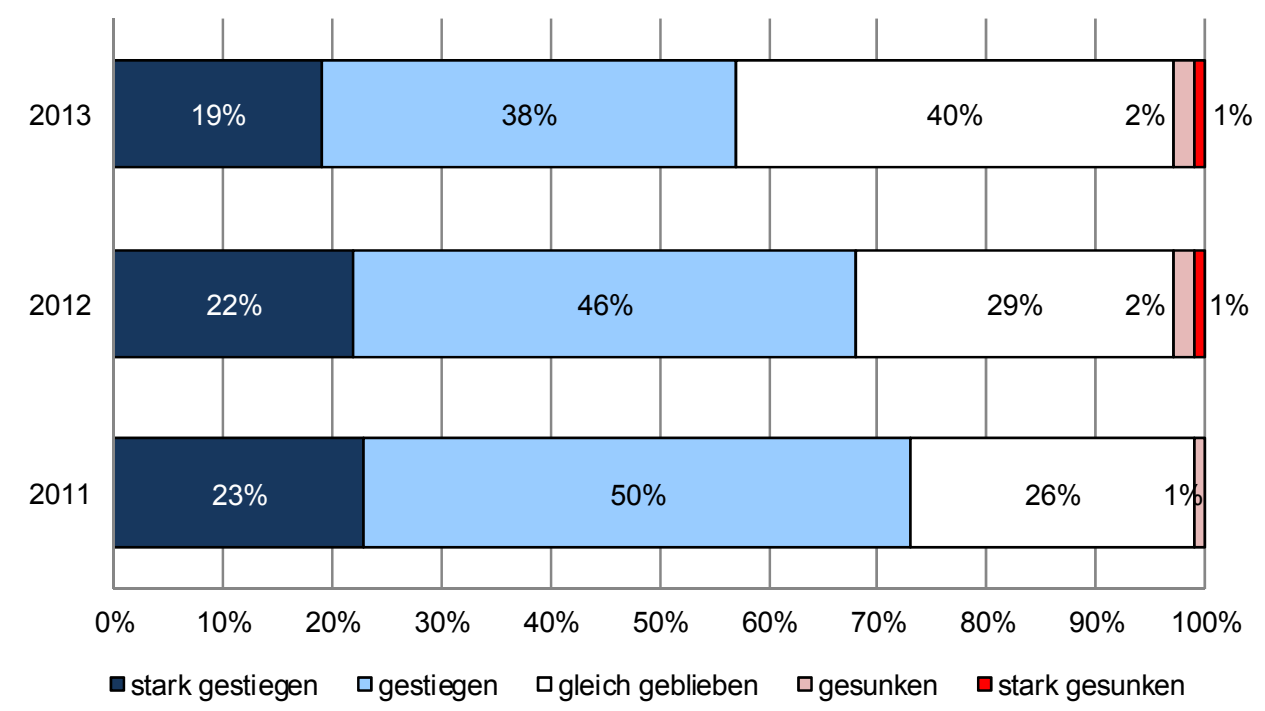

Quelle: Talente entdecken: Praktika für Schülerinnen und Schüler 2013, Feedback Schülerlnnen; $\mathrm{n}=133$ (2011), 164 (2012), 187 (2013)

Aus dem Feedback ist erkennbar, dass die Praktika in den meisten Fällen dazu beigetragen haben, die Vorstellung der Schülerlnnen darüber, welche Aufgaben und Tätigkeiten im Themenfeld von FTI-Organisationen liegen, zu präzisieren und das Informationsniveau über bestimmte Berufe und Ausbildungsmöglichkeiten zu verbessern. Neben Einblicken in die praktische Umsetzung von F\&E-Tätigkeiten lernten die SchülerInnen auch allgemeine Unternehmensabläufe kennen. Darüber hinaus wurden von den SchülerInnen angegeben, dass sie sich neues theoretisches Wissen aneignen konnten sowie bereits erlerntes Wissen, Methoden oder Techniken anwenden konnten. Mehr als die Hälfte der PraktikantInnen (2013 rd. $60 \%$ ) werden die Talente Praktika weiterempfehlen bzw. planen fast $50 \%$ der SchülerInnen auch im nächsten Jahr ein gefördertes Praktikum in Anspruch nehmen.

„Durch die Tätigkeiten während des Praktikums habe ich viel gelernt und konnte mir auch theoretisches Fachwissen aneignen. Das selbständige Arbeiten im Labor ermöglichte mir einen selbstsichereren Umgang mit Forschungsapparaturen und machte mich vertraut mit verschiedensten Arbeitsschritten."

"ICh wollte Physik studieren und dieses Praktikum hat mir den Forschungsalltag und eine etwas technischere Seite von Physik gezeigt und meine Studienwahl bestätigt." 
Negative Aspekte, die in Zusammenhang mit der Beschreibung des Praktikums genannt wurden, waren teilweise unzureichende Betreuungen durch die Praktikumsanbieterlnnen sowie die Einbindung in Bereiche abseits des tatsächlichen Forschungfeldes (z.B. Office-Tätigkeiten, Produktionsbereich).

Für den größten Teil der Unternehmen und Forschungseinrichtungen ist die Programmlinie Praktika für Schülerlnnen eine Möglichkeit, SchülerInnen erste Einblicke in die Forschung und Berufswelt zu gewähren. Das Verhältnis zwischen Formalaufwand und Förderungsleistung wird von fast allen Unternehmen ( $\mathrm{rd}$. $95 \%$ ) als (sehr) ausgewogen bewertet. Dabei ist die PraktikantInnenbörse, sofern sie in Anspruch genommen wurde, auf generellen Anklang gestoßen.

Das Interesse der Unternehmen an der Förderung des ForscherInnennachwuchses wird laut eigenen Aussagen durch die Erfahrungen mit SchülerInnenpraktika gefördert. Rd. 80 \% (dieser Wert ist im Zeitverlauf konstant) der Fördernehmerlnnen geben diesbezüglich an, dass ihr Interesse an der aktiven Nachwuchsförderung stark gestiegen bzw. gestiegen ist.

„Jugendliche können sich durch die Ausschreibung schon früh ein Bild über F\&ETätigkeiten machen, womit evtl. weitere Arbeitskräfte für diesen Bereich begeistert und gewonnen werden können."

Die Unternehmen und Forschungseinrichtungen ziehen nach eigenen Angaben aus dem Anbieten von Praktika konstant hohen, unmittelbaren (2013: 48 \%) bzw. langfristigen Nutzen (2013: 65 \%). Das Praktikumsangebot wird in einem Großteil der Fälle durch die Förderung erst ermöglich, rd. die Hälfte der PraktikumsanbieterInnen sah sich nur aufgrund der Förderung in der Lage, einen Praktikumsplatz anzubieten (2013: $49 \%$, 2012: $48 \%$, 2011: $48 \%$ ), weitere $43 \%$ konnten mehr Praktikumsplätze anbieten, als innen ohne Förderung möglich gewesen wäre (2013: $43 \%$, 2012: $43 \%$, 2011: $44 \%$ ). Hingegen haben nur $8 \%$ bis $9 \%$ der teilnehmenden Forschungstreibenden angegeben, dass sie auch ohne Förderung Praktika in selben Umfang angeboten hätten. Die positive Resonanz spiegelt sich aber auch darin wider, dass jährlich rd. $97 \%$ der geförderten Unternehmen und Forschungseinrichtungen angeben „sicherlich“ bzw. „wahrscheinlich“ auch in Zukunft Praktika für SchülerInnen anzubieten. ${ }^{9}$

„Für uns war es ideal, da wir zusammen mit den Jugendlichen viele Dinge tun konnten, für die wir sonst kaum oder keine Möglichkeit gehabt hätten."

„Die Ausschreibung hat es uns als KMU erst ermöglicht, ein Ferialpraktikum anzubieten. Uns ist es damit gelungen, einem sehr interessierten und begabten Schüler die Mitarbeit in einem Projekt zu ermöglichen und ihn an der Thematik arbeiten zu lassen."

9 Quelle: Talente entdecken: Schülerinnen und Schüler 2013, Feedback Förderungsnehmerlnnen 
Aus Sicht der FFG stellen die SchülerInnenpraktika ein attraktives, unbürokratisches Angebot für FördereinsteigerInnen dar, wobei der Kontakt oft über die Personalabteilungen erfolgt, die nicht unbedingt FFG-erfahren sind. Obwohl das Angebot sehr gut angenommen wird, ist laut FFG doch eine kontinuierliche Informationsverbreitung nötig, da sich die Zielgruppe der SchülerInnen laufend ändert und das Förderangebot immer wieder neuen Personengruppen näher gebracht werden muss.

\subsubsection{Talente regional}

Im Rahmen von Talente regional werden kooperative Projekte gefördert, die Kindern und Jugendlichen ermöglichen, sich über einen längeren Zeitraum in räumlicher Nähe mit FTI-Themen zu beschäftigten.

Tabelle 2 Eckpunkte von Talente regional

\begin{tabular}{|l|l|}
\hline Talente regional & Kurzbeschreibung \\
\hline Ziele & $\begin{array}{l}\text { Die Steigerung des Interesses an FTI und die Förderung der Ausei- } \\
\text { nandersetzung mit Naturwissenschaft/Technik von Kindern/Jugend- } \\
\text { lichen und insbesondere Mädchen. } \\
\text { Die Vernetzung von (vor-)schulischen Bildungseinrichtungen und } \\
\text { Partnern aus Wirtschaft und Forschung. }\end{array}$ \\
\hline Zielgruppen & $\begin{array}{l}\text { Unternehmen, Bildungseinrichtungen (Universitäten, Fachhochschu- } \\
\text { len), Forschungseinrichtungen, Kompetenzzentren, Vereine (För- } \\
\text { dernehmerlnnen). } \\
\text { Kinder und Jugendliche österreichischer, (vor-)schulischer Bildungs- } \\
\text { einrichtungen. }\end{array}$ \\
\hline Inhalt & $\begin{array}{l}\text { Umsetzung praxisnaher FTI-Projekte für Kinder und Jugendliche, um } \\
\text { Einblicke in Tätigkeiten und Berufsbilder im Bereich der Forschung } \\
\text { zu ermöglichen. Projektthemen müssen regionale Verankerungen } \\
\text { aufweisen und geschlechtersensibel umgesetzt werden. }\end{array}$ \\
\hline Einreichverfahren & $\begin{array}{l}\text { Ausschreibungszeiträume von September bis Jänner (2011, 2012, } \\
2013, \text { geplant 2014 September bis Dezember). Förderungswerberln } \\
\text { und Konsortialpartnerlnnen bilden ein Konsortium (mind. ein/eine } \\
\text { wissenschaftliche/r Partnerln und zwei Unternehmenspartnerlnnen } \\
\text { mit Innovationsbezug), welches mit (vor-)schulischen Bildungsein- } \\
\text { richtungen (mind. fünf) als Projektpartnerlnnen kooperiert. }\end{array}$ \\
\hline Faufzeit & $\begin{array}{l}\text { Projektdauer 12 bis 18 Monate } \\
\text { Bis zu } € 60.000 .- \text { (inkl. € 10.000.- Kooperationszuschüsse) }\end{array}$ \\
\hline
\end{tabular}

Schwerpunkt von Talente regional Projekten ist die Entwicklung und Umsetzung verschiedener Bildungsangebote für Kinder und Jugendliche. Durch die Auseinandersetzung mit spannenden FTI-Themen und dem Beruf des Forschers/der Forscherin, Möglichkeiten des Forschens und Experimentierens etc. soll der Zielgruppe Naturwissenschaft/Technik näher gebracht werden. 
Dabei sollen praxisnahe Elemente in den Unterricht eingebracht werden. Auf eine regionale Verankerung ist zu achten.

Ein Konsortium muss aus mindestens einem/einer wissenschaftlichen Partnerln (akademische Einrichtung oder außeruniversitäre Forschungseinrichtung) und zwei UnternehmenspartnerInnen mit Innovationsbezug bestehen. Zudem müssen mindestens fünf (vor-)schulische Bildungseinrichtungen aus drei von vier Bildungsstufen (Kindergarten, Primarstufe, Sekundarstufe I und II) beteiligt sein. Die Konsortialführung oblag den wissenschaftlichen oder wirtschaftlichen Partnerlnnen bzw. in einigen Fällen auch Innovationszentren oder Vereinen.

Im Rahmen eines Talente regional Projekts können max. 10 Kooperationszuschüsse von jeweils 1.000,- Euro vergeben werden. Kooperationszuschüsse bieten (vor-)schulischen Bildungseinrichtungen, die nicht in das Projekt involviert sind, die Möglichkeit, innovative Projekte mit dem Schwerpunkt Naturwissenschaft/Technik durchzuführen. Einen Kooperationszuschuss beantragen PädagogInnen als natürliche Personen direkt bei der Konsortialführung. Damit sollen die Reichweite des Projekts erhöht werden und andere Bildungseinrichtungen vom regionalen Netzwerk profitieren können.

In der ersten Ausschreibung (2011) gelangten 35 Projekte zur Einreichung, 11 davon wurden genehmigt. Vier waren in der Steiermark, je zwei in Tirol und Wien, und jeweils eines in Kärnten, Niederösterreich und Oberösterreich verankert. In der zweiten Ausschreibung (2012) wurden 17 Projekte von 34 eingereichten gefördert. Davon sind vier in Wien, je drei in Oberösterreich und Tirol, je zwei in Kärnten, Niederösterreich und der Steiermark und eines in Salzburg angesiedelt. ${ }^{10}$ In der dritten Ausschreibung (2013) wurden 24 von 50 eingereichten Projekten zur Förderung empfohlen. ${ }^{11}$

\section{Motivation zur Teilnahme}

Grundsätzlich deckte sich die Motivation der ProjektpartnerInnen zur Teilnahme an Talente regional mit den Zielen der Programmlinie, das Interesse und die Begeisterung von Kindern und Jugendlichen für Naturwissenschaft und Technik zu wecken und zu erhöhen. Dabei hatten die Projekte unterschiedliche Themenschwerpunkte und Anknüpfungspunkte: Umweltfragen und Klimawandel, erneuerbare Energien, Ernährung und Nahrungsmittel, Raum- und Luftfahrt, Licht- und Beleuchtung,

10 Die Zuweisung zu den Bundesländern erfolgte von der FFG nach dem Standort der Konsortialführung und nicht nach der Region, in denen die Aktivitäten erfolgten.

11 Die folgenden Ausführungen beziehen sich in erster Linie auf Projekte, die im Rahmen der ersten Ausschreibung gefördert wurden, da diese fast alle mit Ende 2013 abgeschlossen wurden (drei Projekte im Jänner bzw. Februar 2014) und daher Endberichte vorliegen. Die Analysen stützen sich insbesondere auf vier Fallstudien über bereits abgeschlossene Projekte und auf eine OnlineBefragung von PädagogInnen ( $n=53$; hier wurden allerdings Personen befragt, die in Projekte der ersten und zweiten Ausschreibung involviert waren/sind). 
Robotik, Medikamente und Arzneimittel etc. Bei einigen Projekten war ein Genderschwerpunkt zu erkennen.

Die UnternehmenspartnerInnen zielten auch auf den direkten Kontakt zum potentiellen Nachwuchs und wollten den Kindern und Jugendlichen Berufsmöglichkeiten aufzeigen sowie ein realitätsnäheres Bild von technischen Berufen vermitteln, um insbesondere auch Mädchen für diesen Bereich zu begeistern. Aber auch den LehrerInnen sollten ein realistisches Bild von Unternehmen vermittelt und ein Praxisbezug für ihren Unterricht gegeben werden.

Die wissenschaftlichen PartnerInnen sahen im Talente regional Projekt auch eine PR-Maßnahme, um in der jeweiligen Region bei potentiellen StudentInnen und Multiplikatorlnnen bekannter zu werden und ihre Forschungstätigkeiten einem breiteren Publikum näherzubringen. Auch konnten didaktische Konzepte an anderer Klientel und verschiedenen Bildungsstufen erprobt und umgesetzt werden.

Hauptmotive für die involvierten PädagogInnen, an Talente regional teilzunehmen, waren das Interesse am Thema und die Möglichkeit, mit wissenschaftlichen Einrichtungen und Unternehmen (und in eingeschränktem Maße auch mit anderen Bildungseinrichtungen) zu kooperieren. Auch sollte das Interesse an Naturwissenschaft und Technik von Kindern und Jugendlichen im Allgemeinen und Mädchen im Besonderen geweckt werden und dies durch innovatives Arbeiten und praktische Erfahrungen, die Abwechslung und Bereicherung für den Unterricht bringen, unterstützt werden. Einige Bildungseinrichtungen konnten mit Talente regional auch an deren Schwerpunkt „Forschen“ oder „Naturwissenschaft" anknüpfen bzw. sahen darin eine PR-Maßnahme.

Zur Teilnahme der PädagogInnen an dem Talente regional Projekt kam es in erster Linie durch die direkte Ansprache durch die Projektleitung (49\%) oder die wissenschaftlichen (23\%) PartnerInnen. In etwas mehr als $10 \%$ der Fälle war die gemeinsame Initiative infolge eines Vorgängerprojektes entstanden. Weitere 13\% gaben andere Veranlassungen für eine Projektteilnahme an, wobei vorwiegend persönliche Kontakte angeführt worden sind (siehe Grafik 14 im Anhang).

Über persönliche Kontakte erfuhren die meisten PädagogInnen (70 \%) auch über Talente regional. Rd. ein Viertel der Befragten wurde von der Schulleitung oder KollegInnen auf Talente regional hingewiesen. Das Science Center Netzwerk (6\%) bzw. Messebesuche (2\%) waren hingegen kaum ausschlaggebend für die Wahrnehmung der Programmlinie durch die PädagogInnen (siehe Grafik 15 im Anhang).

Die teilnehmenden PädagogInnen waren in den meisten Fällen schon „vorbelastet“. Fast 70 \% gaben an, schon früher Initiativen zur Förderung des Interesses des Nachwuchses an Naturwissenschaft und Technik gesetzt zu haben. Diese reichen von Exkursionen, Freifächer Forschen \& Experimentieren über kleinere Projekte mit anderen Einrichtungen bis zur Teilnahme an anderen Förderprojekten (z. B. Sparkling Science). 


\section{Projektinhalte}

Grundsätzlich können zwei Projektarten unterschieden werden. Projekte, in denen die einzelnen Aktionen gezielt ineinander greifen und auch sehr konkrete „Produkte" erzeugt werden (beispielweise die Erarbeitung eines Beleuchtungskonzepts bis zur Installierung oder der Bau von Motorseglern - siehe Fallstudien „LIchtQuadrat“ oder "fly DOO“ im Anhang) und Projekte, bei denen die Themen als losere Klammer dienen, um verschiedenste Aktionen in den verschiedenen Bildungseinrichtungen durchzuführen (siehe z.B. Fallstudie „Technologykids“ im Anhang).

In den Vorbereitungsphasen der Projekte fanden in erster Linie Auftaktveranstaltungen zur Information der involvierten AkteurInnen (PädagogInnen, Eltern etc.) statt. Ebenso wurden in einigen Fällen Workshops für PädagogInnen angeboten, damit diese in der Folge das Projekt mit den Kindern und Jugendlichen im Unterricht vorbereiten konnten.

Im Zentrum aller Projekte stand die Begegnung der SchülerInnen und Kleinkinder mit Forscherlnnen, meist im Rahmen von Aktionstagen, an denen gemeinsam experimentiert, geforscht, gemessen, analysiert und ausprobiert wurde. Zumeist kamen die ForscherInnen in die Bildungseinrichtungen, stellten entsprechendes Material (z. B. Mikroskope, Experimentierkoffer etc.) zur Verfügung und leiteten die Kinder an, selbstständig zu experimentieren. In einigen Fällen konnten die Kinder auch die Forschungslabors oder die Werkstätten der UnternehmenspartnerInnen besuchen. Dabei wurden die Forschungsfragen und in der Folge die praktischen Übungen altersgerecht adaptiert und gestaltet. Diese Adaptierung der wissenschaftlichen Inhalte und die Aufbereitung für die verschiedenen Altersgruppen stellt laut PädagogInnen eine große Herausforderung dar. In einigen Projekten wurden auch zu Peer-Tutoring-Aktivitäten durchgeführt, bei denen ältere SchülerInnen jüngere Kinder anleiteten.

Den Abschluss des Projekts bildete in den meisten Fällen ein Forschungsfest, bei dem die Kinder und Jugendlichen ihre Projekte einem breiteren Publikum präsentieren konnten. In einigen Projekten wurden Wettbewerbe organisiert (z. B. „Wer baut den besten Energiespeicher") oder Preise für die besten Schülerlnnen vergeben (z. B. Aufenthalt im NASA Space Camp).

In Hinblick auf inhaltliche Erfolgsfaktoren spiegelt die Meinung der PädagogInnen die Ansichten der anderen in den Projekten involvierten Personen (siehe Grafik 5). Auch für die PädagogInnen (91 \% sehr wichtig und weitere $6 \%$ wichtig) steht das eigene Experimentieren im Zentrum, um Kinder und Jugendliche für Naturwissenschaft und Technik zu begeistern. Dazu braucht es die Anwendung von passenden Unterrichtsmaterialien (85\% (sehr) wichtig). Aber auch der persönliche Kontakt zu Forscherlnnen wird von mehr als $70 \%$ der PädagogInnen als sehr wichtig (und weiteren $20 \%$ als wichtig) erachtet und steht oftmals im Zusammenhang mit dem Besuch einer wissenschaftlichen Einrichtung (88 \% (sehr) wichtig). Als Erfolgsfak- 
toren werden zudem von mehr als $80 \%$ der Befragten der Besuch innovativer Unternehmen und die Vorbereitung im Unterricht genannt. Rd. drei Viertel sehen darüber hinaus den Erwerb unterstützender Anschaffungen, die Ergebnispräsentation im Rahmen eines Events und Möglichkeit der Formulierung eigener Forschungsfragen als Schlüssel zum Erfolg.

Grafik 5 Wichtigkeit von Faktoren, um Kinder/Jugendliche für Naturwissenschaft/Technik zu begeistern

\section{Eigenes Experimentieren}

Persönlicher Kontakt zu Forscherlnnen/Entwicklerlnnen/Wissenschafterlnnen

Erhalt/Anwendung von passendem Unterrichtsmaterial (z.B. Versuchsanleitungen)

Besuch einer wissenschaftlichen Einrichtung Vorbereitung im Unterricht

Erwerb von unterstützenden Anschaffungen (z.B. Mikroskop)

Besuch eines innovativen Unternehmens

Möglichkeit, Forschungsfragen selbst zu formulieren/zu beantworten

Möglichkeit, Ergebnisse im Rahmen eines Events zu präsentieren

Coaching/Mentoring/Peer-Tutoring durch ältere SchülerInnen

Gendersensible Gestaltung des Projekts

Einbeziehung der Eltern
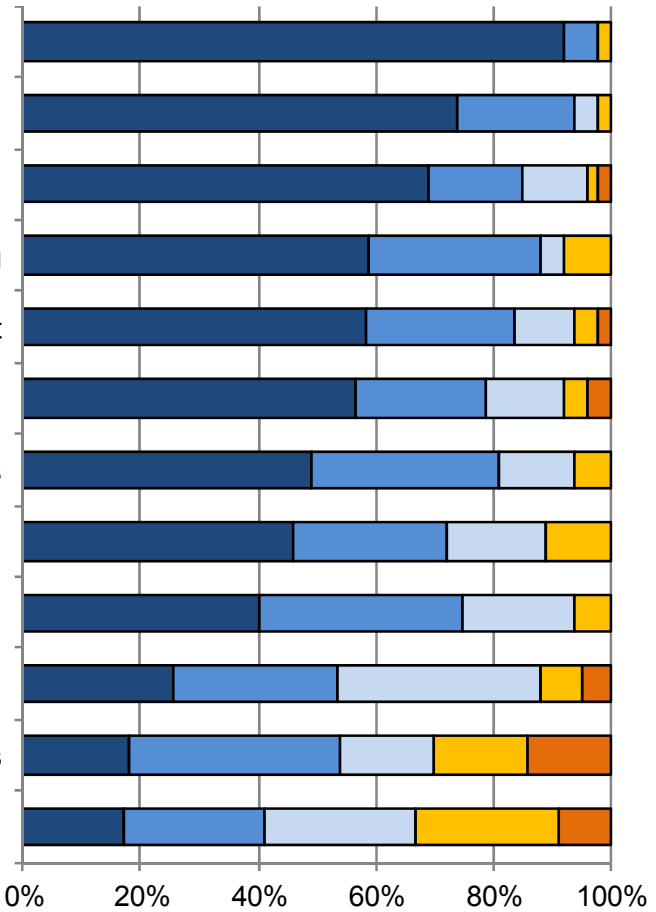

1 sehr wichtig $\square 2$ wichtig $\square 3$ eher wichtig $\square 4$ weniger wichtig $\quad \square 5$ unwichtig

Quelle: Online-Befragung der in Talente regional involvierten PädagogInnen, $n=49$

\section{Kooperationen}

Die Zusammenarbeit im Rahmen der Talente regional Projekte wird von allen befragten Personen sehr positiv hervorgehoben. Auch die Zufriedenheit der Pädagoglnnen mit der Kooperation im Konsortium ist mit 90 \% (sehr zufrieden bzw. zufrieden) äußerst hoch (siehe Grafik 6). Vor allem die Projektleitung stößt auf sehr gute Resonanz, gefolgt von der Zusammenarbeit mit den wissenschaftlichen und wirtschaftlichen PartnerInnen. Vergleichsweise schlechter wird die Kooperation mit den anderen Bildungseinrichtungen bewertet, obwohl sich auch hier drei Viertel der PädagogInnen (sehr) zufrieden zeigen. 


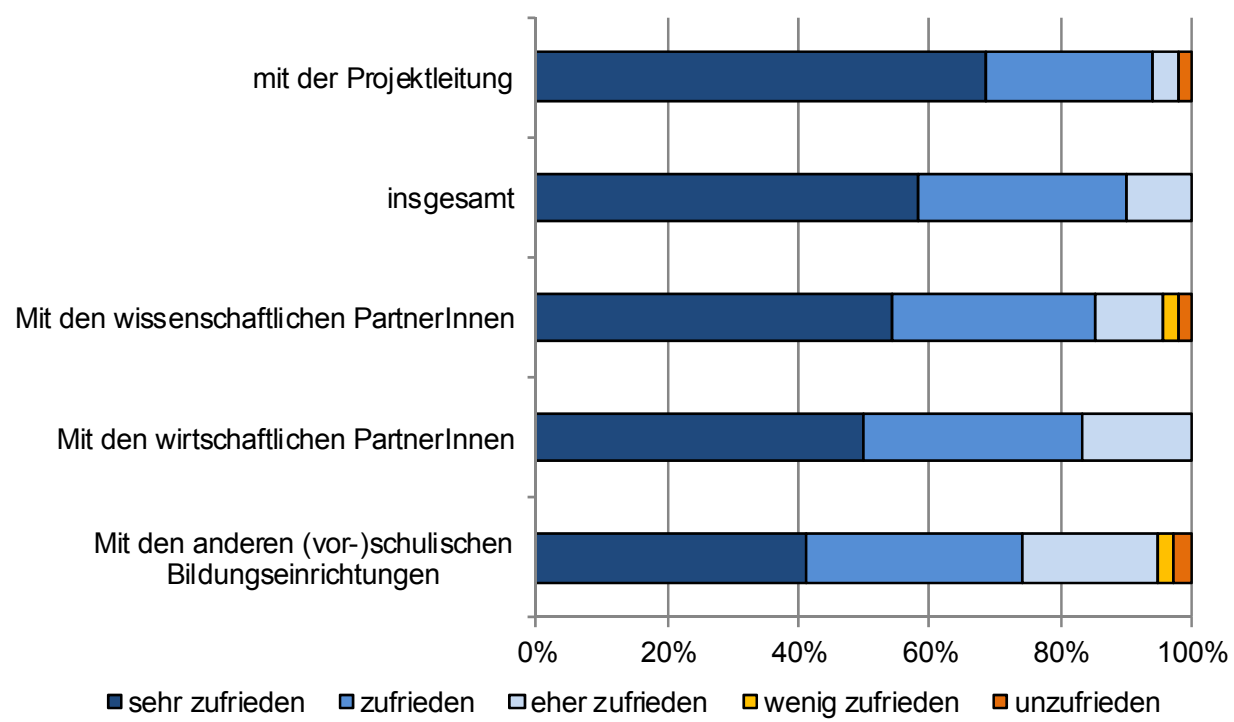

Quelle: Online-Befragung der in Talente regional involvierten Pädagoglnnen, $n=36$

Meist laufen die Fäden bei der Projektleitung zusammen, die oftmals auch die Initiative zum Projekt gesetzt hat. In den Konsortien haben zumeist ein Teil der wissenschaftlichen und wirtschaftlichen PartnerInnen schon in Vorprojekten kooperiert und/oder die handelnden Personen sind durch persönliche Netzwerke verbunden. Dies trifft weniger auf die BildungspartnerInnen zu. Rd. drei Viertel der Pädagoglnnen hatten davor keinen Kontakt mit den ProjektpartnerInnen (siehe Grafik 16 im Anhang). Am ehestens bestand noch Kontakt zu anderen Bildungseinrichtungen, ein Viertel berichtet davon, sich schon vor dem Projekt mehrmals mit anderen Bildungseinrichtungen ausgetauscht $\mathrm{zu}$ haben. Auch mit den wissenschaftlichen PartnerInnen und der Projektleitung hatte rd. ein Viertel der PädagogInnen einmal oder mehrmals Kontakt. Dies geschah meist im Rahmen von Vorgängerprojekten ( $v$ a. mit der Projektleitung), über längerfristige Kooperationen (hier v. a. mit den wissenschaftlichen PartnerInnen) oder über Veranstaltungen, Fortbildungen oder weil die PädagogInnen in mehreren Einrichtungen unterrichten (mit anderen Bildungseinrichtungen).

Für eine gut funktionierende Kooperation erachten über $90 \%$ der PädagogInnen folgende Faktoren für entscheidend: Das Engagement der involvierten Personen und ProjektpartnerInnen, klare Verantwortlichkeiten und das Vorliegen eines Zeitplan. Über $60 \%$ beurteilen die laufende Kommunikation und regelmäßige Projektmeetings entscheidend für eine erfolgreiche Kommunikation (siehe Grafik 7). Adhoc Meetings werden vergleichsweise weniger wichtig eingestuft. 
Grafik 7 Faktoren für eine gut funktionierende Kooperation

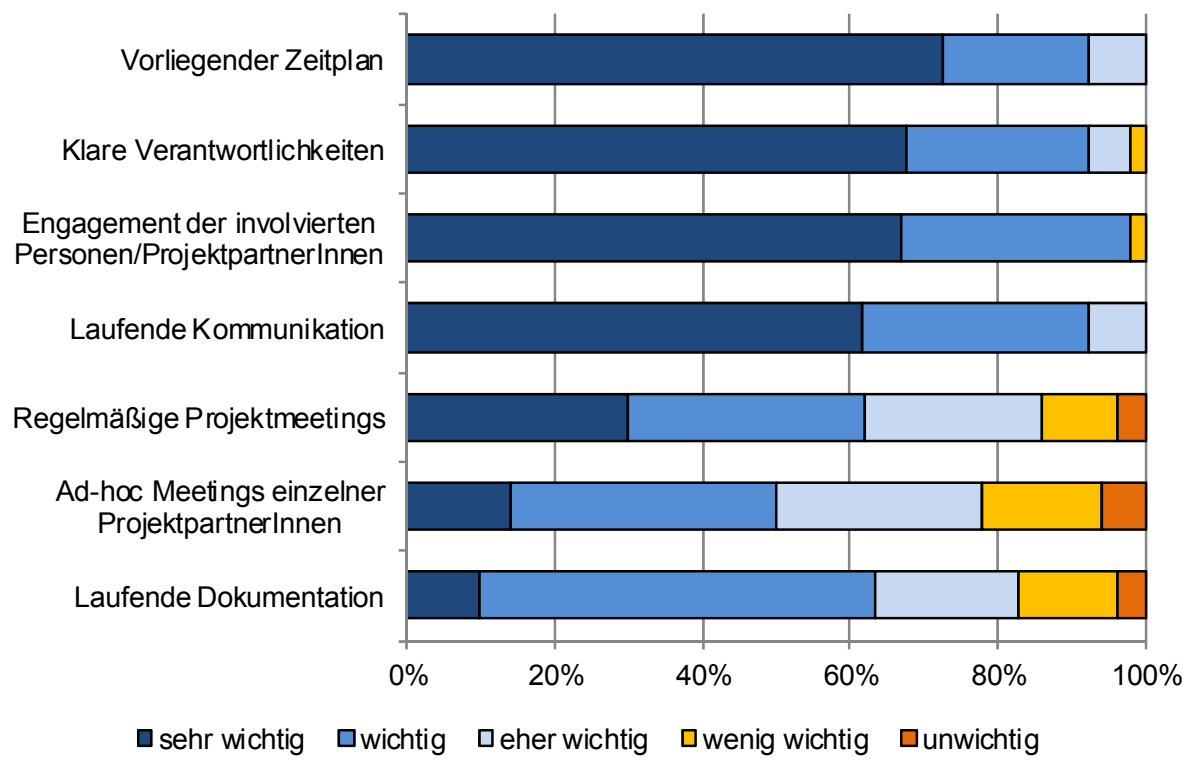

Quelle: Online-Befragung der in Talente regional involvierten Pädagoglnnen, n=52

Damit decken sich die Meinungen der PädagogInnen mit jenen der anderen ProjektpartnerInnen. Eine klare Rollenaufteilung und eine transparente Kommunikation werden von den Interviewten ebenso genannt wie das persönliche Engagement der Involvierten. Aber auch das gemeinsame Verständnis der Ziele und Inhalte des Projekts werden als wichtig für eine gelungene Kooperation gesehen.

Die größte Herausforderung in Hinblick auf die Kooperation scheint die Integration des Projekts in das laufende Schuljahr und in den Schulalltag zu sein. Während die Abstimmung mit Kindergärten und Volksschulen noch leichter fällt, weil diese zeitlich flexibler sind, wird es ab der Sekundarstufe I schwieriger, da hier ein enges zeitliches Korsett und immer auch ein Abstimmungsbedarf mit anderen LehrerInnen gegeben ist, sobald die Aktivitäten über eine Unterrichtsstunde hinausgehen. Daher verwundert es auch nicht, dass die PädagogInnen im Zeitmanagement und in der gemeinsamen Terminfindung und Koordination die größten Probleme in der Zusammenarbeit sehen. Auch bei der Beurteilung des Projekts in seiner Gesamtheit erwiesen sich für die PädagogInnen ihre eigenen Zeitressourcen (67\%) und die Integration des Projekts in das laufende Schuljahr (63\%) als größte Hürde, die es zu überwinden galt. Rd. die Hälfte (52\%) empfand auch die Integration des Projekts in den Unterricht und die administrativen Erfordernisse als herausfordernd (46\%). 
Obwohl trotz dieser schwierigen Rahmenbedingungen das Engagement der PädagogInnen gelobt wird, berichten einige ProjektleiterInnen doch vom hohen Bedarf persönlicher und intensiver Betreuung der Lehrerlnnen und von einer teilweise „konsumierenden Haltung mancher PädagogInnen“.

Während die Projektpartnerlnnen aus Wissenschaft und Wirtschaft oftmals ihre Partnerschaft in einem neuen Talente regional Projekt erneuern ${ }^{12}$, dürften die Bildungseinrichtungen in den Projektteams häufiger wechseln. Nur $9 \%$ der Pädagoglnnen gaben an, in einem Folgeprojekt mit PartnerInnen aus dem Talente regional Projekt zu arbeiten, weitere $26 \%$ befinden sich in der Planungsphase für ein Folgeprojekt. Ein Viertel der Befragten gibt auch an, auch außerhalb von Talente regional mit einem oder mehreren der PartnerInnen zusammenzuarbeiten, $8 \%$ planen dies. Diese Kooperationen betreffen in erster Linie Schulpartnerschaften, Weiterbildungsaktivitäten oder Projektarbeiten.

\section{Wirkungen}

Die Projektpartnerlnnen berichten in Hinblick auf die teilnehmenden Kinder und Jugendlichen v.a. von großer Begeisterung. Insbesondere auch Kinder, die durch ihr soziales Umfeld weniger Möglichkeiten erhalten, solche Eindrücke zu sammeln, zeigen sich sehr engagiert und dankbar. Die Zielgruppe erhalte einen guten Einblick in die Forschung und Berufsbilder in Naturwissenschaft und Technik. Forschung und Wissenschaft werden erfahrbar gemacht. Teilweise wird auch berichtet, dass bei einigen konkrete Berufswünsche in diese Richtung wachsen bzw. die Entscheidung beispielsweise für einen technischen Lehrberuf oder ein einschlägiges Studium gefallen ist.

„Bei manchen gibt das einen Kick und da entsteht Begeisterung.“

„Da entsteht plötzlich eine Dynamik ... und da geht es schon los, da lebt es dann."

„Und da höre ich dann: ,Ich will sowas selbst machen können'.“

„Dann ändert sich das Bild, dass sie Großteils haben, wo sie glauben, in so ein Unternehmen geht man rein, da ist es dreckig, da ist es laut, da hat man schwarze Fingernägel, und dann sehen sie, dass wir mit High-Tech Maschinen arbeiten und Sauberkeit das oberste Gebot ist."

12 Im Rahmen der Fallstudien berichten drei Projektteams von einer neuerlichen Zusammenarbeit in der zweiten bzw. dritten Ausschreibung. 
Auch die PädagogInnen wurden hinsichtlich möglicher Wirkungen auf die Kinder und Jugendlichen befragt ${ }^{13}$. Die größte Zustimmung erhielten dabei die Aussagen „Die Kinder/Jugendlichen haben ihre Kenntnisse im Bereich Technik/Naturwissenschaft erweitert" und „Die Kinder/Jugendlichen haben einen Einblick in die Tätigkeit eines Forschers/einer Forscherin gewonnen“. Über 60 \% berichten auch davon, dass das Interesse der teilnehmenden Kinder/Jugendlichen an der Naturwissenschaft/Technik im Allgemeinen gestiegen ist, während die Interessenssteigerung bei den Mädchen im Besonderen seltener beobachtet wurde (siehe Grafik $17 \mathrm{im}$ Anhang).

Das Projekt bot laut PädagogInnen neben der Steigerung des Interesses an Naturwissenschaft und Technik auch die Möglichkeit, einerseits besonders interessierte SchülerInnen gezielt zu fördern, andererseits aber auch Kinder einzubinden, denen sonst nur wenige zusätzliche Aktivitäten geboten werden. Als das Besondere am Projekt bezeichneten die Lehrerlnnen den direkten Kontakt und das Arbeiten mit den Forscherlnnen, was einen großen Praxisbezug mit sich brachte und Einblick in unbekannte Berufsfelder erlaubte.

Für die wissenschaftlichen PartnerInnen bot das Talente regional Projekt die Gelegenheit, ihre Tätigkeiten einem breiteren Publikum zu vermitteln und in der Region - auch als Ausbildungsstätte - bekannter zu werden. Dabei agieren die Kinder als MultiplikatorInnen zu den Eltern. Dies trägt auch zur Verbreitung der in den Projekten behandelten Themen (Umweltschutz, Energiesparen etc.) bei. Die beteiligten (jungen) Forscherlnnen erhielten vielfach einen Motivationsschub und konnten auch wichtige Erfahrung in Hinblick auf die Lehre (im Falle von Universitäten und Fachhochschulen) sammeln. Materialien und Programme wurden erarbeitet, die nun auch in anderen Schulen verwendet werden können.

Die beteiligten Unternehmen konnten sich als engagierter ArbeitgeberInnen positionieren und erhielten direkten Kontakt zu potentiellen zukünftigen Nachwuchskräften sowie insbesondere zu Mädchen. Für die Region bedeutet ein solches Projekt die Gelegenheit, sich als Forschungsstandort und innovative Region darzustellen.

Auch bei den PädagogInnen zeigte die Teilnahme an dem Talente regional Projekt entsprechende Wirkungen. Diese konnten v.a. ihre Kenntnisse im Bereich Naturwissenschaft/Technik erhöhen und planen in Zukunft mehr Projektarbeit in den Unterricht zu integrieren. Durch die Teilnahme am Projekt haben die PädagogInnen Methoden kennengelernt, um das Interesse an Naturwissenschaft/Technik bei den Kindern/Jugendlichen zu erhöhen und konnten auch ihre allgemeinen methodischen Kenntnisse erweitern sowie Methoden gendersensibler Unterrichtsgestaltung kennenlernen (siehe Grafik 18 im Anhang).

13 Die PädagogInnen sollten Aussagen in Hinblick auf mögliche Wirkungen auf die teilnehmenden Kinder und Jugendlichen auf einer Notenskala von 1 bis 5 hinsichtlich ihrer Zustimmung beurteilen (stimme sehr zu bis stimme nicht zu). 
Einen Mehrwert des Projekts stellte für die PädagogInnen auch die Bereitstellung von Hilfsmitteln und Materialien (z. B. Mikroskop) dar, die sonst im Unterricht oder im Kindergarten nicht zur Verfügung stehen. Zudem konnten im Projekt neue Kontakte zu unterschiedlichsten Einrichtungen geknüpft werden.

\section{Allgemeine Beurteilung der Programmlinie}

Die interviewten ProjektpartnerInnen sehen die Programmlinie Talente regional nicht nur sehr geeignet, um Kinder und Jugendliche für Naturwissenschaft und Technik zu begeistern, sondern erachten derartige Förderungen auch als absolut notwendig, um das Interesse der Zielgruppe zu steigern. Mit solchen Projekten werde der natürliche Wissensdrang der Kinder unterstützt und diese können als MultiplikatorInnen agieren, was die Verbreitung der „Message“ unterstützt.

Als Stärke der Programmlinie werden die Offenheit bezüglich der Themen und Herangehensweisen genannt sowie die Langfristigkeit der Projekte. Von großer Relevanz sind auch die hohe Praxisorientierung sowie die zahlreichen Interaktionsmöglichkeiten von Forscherlnnen und Kindern/Jugendlichen. Dabei sei jedoch entscheidend, dass Personen involviert sind, die ihre Begeisterung für die Forschung auch zeigen. Zudem sollte das Projekt griffige Ideen enthalten und inhaltlich homogen sein.

„Über ein Schuljahr mit Klassen zusammenarbeiten, hat sicher eine andere Tiefe als punktuelle Maßnahmen, weil es auch den Raum bietet, auszuprobieren“

„Da springt dann der Funke auf die Kinder über.“

„Wenn man mehr als $100 \%$ geben könnte... es ist wirklich optimalst."

Aus Sicht der PädagogInnen ist ein strukturiertes Projektmanagement Grundvoraussetzung für den Erfolg eines Talente regional Projekts (siehe Grafik 8). Alle Befragten sehen dies als einen (sehr) wichtigen Erfolgsfaktor. Zudem ist die Kooperation mit wissenschaftlichen Einrichtungen (96\%) und mit Unternehmen (92 \%) für die PädagogInnen von (sehr) großer Bedeutung, da sie die Voraussetzung für eine hohe Praxisorientierung darstellt. Die Schulung der involvierten PädagogInnen wird von drei Viertel als (sehr) wichtig erachtet, die Kooperation mit anderen Bildungseinrichtungen scheint hingegen für die befragten PädagogInnen etwas weniger bedeutend für den Erfolg, dennoch beurteilen diese Zusammenarbeit noch immer $60 \%$ als (sehr) wichtig. 


\section{Grafik 8 Wichtigkeit der Faktoren für den Erfolg eines Talente regional Projekts}

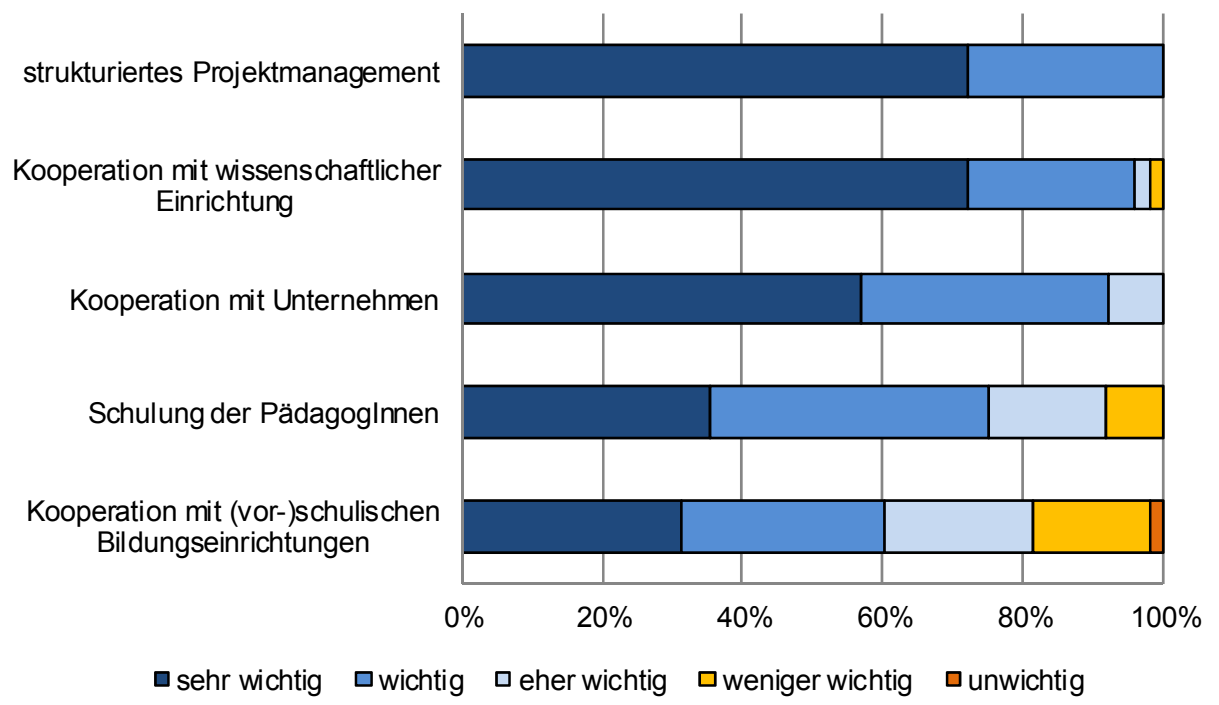

Quelle: Online-Befragung der in Talente regional involvierten PädagogInnen, $n=51$

Obwohl die Einbindung der verschiedenen PartnerInnen als wichtig erachtet wird, scheint diese auch die größte Herausforderung zu sein. Damit geht ein großer Koordinationsaufwand einher, der sich nur ungenügend im Gesamtbudget bzw. im Anteil des Projektmanagements von max. $20 \%$ widerspiegelt. Insbesondere die Koordination mit den Schulen (Integration in den Schulalltag und in die Planung des Schuljahres, administrative Erfordernisse bezüglich außerschulischer Aktivitäten, Lehrplan versus Projektinhalte etc.) erweist sich als sehr schwierig. In diesem Zusammenhang werden auch die Kooperationszuschüsse genannt, die zusätzlichen koordinativen Aufwand erzeugen.

Inhaltlich herausfordernd ist es für die Projektbeteiligten, den Kindern und Jugendlichen unterschiedlichster Altersstufen adäquat zu begegnen und die Forschungsthemen altersgruppenspezifisch aufzubereiten. Die Integration von mindestens drei Bildungsstufen erweist sich hier als erschwerend.

Trotz dieser Schwierigkeiten ist die Programmlinie Talente regional für die Befragten derart attraktiv, dass drei von vier befragten Konsortien in ähnlicher Konstellation in der zweiten bzw. dritten Ausschreibungsrunde eingereicht haben. Die Programmlinie wird als „eine tolle, aber sehr ambitionierte Sache“ beschrieben.

Auch die Bilanz der teilnehmenden PädagogInnen ist eine sehr positive. Alle stimmen der Aussage zu, dass sich die Teilnahme am Projekt für die jeweilige Bildungseinrichtung gelohnt hat. Fast drei Viertel können sich vorstellen, wieder an einem ähnlichen Projekt teilzunehmen und über $90 \%$ empfehlen Kolleglnnen die Teilnahme an einem Talente regional Projekt weiter. 


\subsection{Talente nützen: Chancengleichheit}

\subsubsection{FEMtech Forschungsprojekte}

Die Programmlinie FEMtech Forschungsprojekte zielt auf die Unterstützung von FTI-Projekten mit genderrelevanten Inhalten ab. Durch die Berücksichtigung unterschiedlicher Lebensrealitäten von Frauen und Männern streben die Forschungsvorhaben zielgruppenrelevante Innovationen und die Schaffung neuer Marktpotenziale an.

Tabelle 3 Eckpunkte von FEMtech Forschungsprojekte

\begin{tabular}{|c|c|}
\hline $\begin{array}{l}\text { FEMtech } \\
\text { Forschungsprojekte }\end{array}$ & Kurzbeschreibung \\
\hline Ziele & $\begin{array}{l}\text { Initiierung und Unterstützung von FEI-Vorhaben mit genderrelevan- } \\
\text { ten Projektinhalten. }\end{array}$ \\
\hline Zielgruppen & $\begin{array}{l}\text { FTI-Unternehmen, Hochschulen und außeruniversitäre Forschungs- } \\
\text { einrichtungen }\end{array}$ \\
\hline Inhalt & $\begin{array}{l}\text { Förderung von FEI-Forschungsvorhaben, deren Forschungsgegen- } \\
\text { stand die unterschiedlichen Lebensrealitäten von Männern und } \\
\text { Frauen abdeckt. Die Genderrelevanz der Projektinhalte unterstützt } \\
\text { Innovationen sowie die Schaffung neuer Marktpotenziale. }\end{array}$ \\
\hline Einreichverfahren & $\begin{array}{l}\text { Einreichung nach Ausschreibung (2011, 2012, } 2013 \text { - jeweils Okto- } \\
\text { ber bis Jänner), geplanter Ausschreibungszeitraum } 2014 \text { Oktober } \\
\text { bis Jänner). }\end{array}$ \\
\hline Laufzeit & Mindestens ein Jahr bis maximal drei Jahre. \\
\hline Förderhöhe & $\begin{array}{l}\text { Bis zu } € 300.000 \text {.- pro Projekt; Die Förderungsquote beträgt max. } \\
70 \% \text { (Einzelprojekte) bzw. } 80 \% \text { (Kooperative Projekte) abhängig } \\
\text { von der Forschungskategorie und des Organisationstyps. }\end{array}$ \\
\hline
\end{tabular}

Im Zentrum der FEMtech Forschungsprojekte stehen Forschungsvorhaben aus dem Bereich der angewandten Forschung, die genderrelevante Inhalte abhandeln. Genderrelevanz wird dabei als die Inklusion vielfältiger Lebensrealitäten in die Entwicklung von Produkten, Prozessen und Dienstleistungen definiert, die geschlechterunterschiedliche Bedürfnisse und Nutzerverhalten berücksichtigen. Ein wesentliches Kriterium der Projektförderung ist die vorhandene Genderkompetenz der forschenden Unternehmen und Forschungseinrichtungen.

In den bisherigen Ausschreibungsrunden wurden jeweils acht FEMtech Forschungsprojekte gefördert, wobei im Jahr 201120 Ansuchen und im Jahr $2012^{14}$ 30 Projektvorhaben eingereicht wurden. Die Bewilligungsquoten liegen dementsprechend bei $40 \%$ bzw. $27 \%$. Im Zuge der dritten Ausschreibung sind 36 Projek-

14 Vgl. FFG-Monitoringsdaten 
te zur Beurteilung eingegangen, die endgültigen Förderbescheide stehen jedoch zum Zeitpunkt der Zwischenevaluierung noch nicht fest. Anhand der steigenden Anzahl der Förderansuchen lässt sich ein zunehmendes Interesse an der Umsetzung genderrelevanter Forschungsthemen ableiten. Die steigende Nachfrage übertrifft dabei die Zielgröße von 20 bis 30 eingereichten Förderprojekten, allerdings liegt die Zahl der tatschlich geförderten Forschungsprojekte leicht unterhalb des angestrebten Wertes von 10 bis 12 geförderten Projekten. Diese Ergebnisse sind jedoch in Relation zu den vorhandenen Fördermitteln zu betrachten. Während im Zuge der ersten Ausschreibung lediglich rd. zwei Drittel der verfügbaren Gelder auch ausbezahlt wurden, kam es im Rahmen der zweiten Ausschreibung zur vollständigen Mittelausschöpfung ${ }^{15}$.

Betrachtet man die Verteilung der geförderten Organisationstypen der FEMtech Forschungsprojekte, so zeigt sich ein leichtes Übergewicht zu Gunsten von Unternehmen. Insgesamt war mehr als die Hälfte der FördernehmerInnen (erste und zweite Ausschreibung) dem Unternehmensbereich zuzurechnen, während es sich bei rd. einem Viertel um außeruniversitäre Forschungseinrichtungen handelte. Weitere $16 \%$ stammen aus dem akademischen Forschungsbereich (Universitäten und FHs) bzw. waren 4\% Sonstige (z.B. EinzelforscherInnen) involviert. Die Fördernehmerlnnen sind dabei zum größten Teil in Wien lokalisiert (78 \%) sowie in der Steiermark und in Niederösterreich. Darüber hinaus wurden 15 der 16 Projekte in Form von kooperativen Forschungstätigkeiten umgesetzt.

Die themenoffene Gestaltung der FEMtech Forschungsprojekte drückt sich in den bisherigen Ausschreibungsrunden durch recht heterogene Anwendungsfelder der geförderten FTI-Projekte aus. Die unterstützten Forschungsvorhaben der Jahre 2011 und 2012 waren etwa in den Bereichen Verkehr und Mobilität, IKT, Architektur und Bautechnik sowie Humanmedizin angesiedelt ${ }^{16}$. Diese Themenoffenheit wird von den befragten Projektteilnehmerlnnen als einer der wesentlichen Programmvorteile gesehen, da sie die Möglichkeit bietet, in vielfältigen, gesellschaftlich relevanten Bereichen genderrelevante Fragestellung zu adressieren und entsprechende Lösungsansätze zu erarbeiten.

Für rd. $90 \%$ der Unternehmen und Forschungseinrichtungen war die Partizipation in der Programmlinie FEMtech Forschungsprojekte nicht die erste Förderung durch die FFG. Dementsprechend zeigten sich die befragten FördernehmerInnen der ersten Ausschreibung von FEMtech Forschungsprojekte mit den Fördermodalitäten vertraut.

15 Programmdokument und Sonderrichtlinien Talente - Der Förderschwerpunkt des BMVIT

16 FEMtech Forschungsprojekte Projektanträge 


\section{Motivation zur Einreichung}

Die Projekte der untersuchten FördernehmerInnen, die als Fallstudie der FEMtech Forschungsprojekte erfasst wurden (siehe Anhang), waren überwiegend Fördernehmerlnnen, die sich im Zuge ihrer Forschungstätigkeiten bereits mit Genderthemen auseinandergesetzt haben. Dies drückt sich unter anderem in der bestehenden Expertise (unternehmensinterne GenderexpertInnen, Genderbeauftragte) und der Genderorientierung (ausgeglichene Teamzusammensetzung bzw. Rekrutierungsmaßnahmen, Fortbildungsmaßnahmen) der geförderten Organisationen bzw. Konsortien aus. Die Betrachtung genderrelevanter Inhalte wird dabei tlw. explizit als Forschungsschwerpunkt im Angebotsportfolio der Organisationen erwähnt. Die Bearbeitung genderrelevanter Forschungsfelder wird von den befragten FördernehmerInnen einerseits mit dem hohen gesellschaftlichen Nutzen begründet, und lässt sich andererseits auf die zunehmende Wahrnehmung solcher Themen auch in den technischen Forschungsgebieten zurückführen.

Wenig überraschend stand in den untersuchten Forschungs- und Innovationsvorhaben der FEMtech Forschungsprojekte die Genderrelevanz von Beginn an im Zentrum der thematischen Fragestellungen bzw. leitete sich die technische Fragestellung aus dem genderrelevanten Bedürfnis ab. Trotz des hohen gesellschaftlichen Nutzens, der in Zusammenhang mit den angestrebten genderorientierten Forschungsvorhaben steht, war die Projektabwicklung von der Akquisition von Fördergeldern abhängig. Für die untersuchten Projekte bzw. die forschenden Organisationen gilt, dass ohne Unterstützung durch die FEMtech Forschungsprojekte die Umsetzung auf Grund unzureichender finanzieller Eigenmittel nicht möglich gewesen wäre.

„Der Projektumfang und die dadurch anfallenden Kosten konnten nur bewältigt werden, weil wir die Förderung erhalten haben."

„Ohne Förderung wäre es nicht zur Umsetzung gekommen, etwaige Folgeprojekte wären ebenfalls vom Zugang zu externen Mitteln abhängig."

\section{Relevanz und Genderaspekt FEMtech Forschungsprojekte}

In Hinblick auf die Relevanz der FEMtech Forschungsprojekte lässt sich nach Aussagen der FördernehmerInnen festhalten, dass eine Angebotslücke der Förderlandschaft, nämlich die Berücksichtigung des Genderaspekts als zentraler Forschungsinhalt in technischen Forschungsvorhaben, erfolgreich geschlossen werden konnte. Häufig herrscht vor allem in technisch-naturwissenschaftlichen Bereichen der Forschung und Entwicklung einerseits noch eine unzureichende Sensibilisierung bezüglich genderrelevanter Fragestellung vor. Dadurch trägt die Einführung dieser speziellen Programmlinie dazu bei, die Wahrnehmung unterschiedlicher Lebensrealitäten in oftmals männerdominierte Forschungsfelder einzubezie- 
hen. Andererseits wird der Genderaspekt in anderen Forschungsförderungen zwar teilweise erwähnt, meistens jedoch nur als ein Nebenaspekt erachtet und erfährt daher keine ausreichende Betrachtung im Zuge der angestrebten Forschungstätigkeiten. Diese gezielte Ausrichtung der geförderten FTI-Aktivitäten auf genderrelevante Forschungsfragen haben die Etablierung der FEMtech Forschungsprojekte begünstigt und zu einer hohen Bekanntheit in der relevanten Zielgruppe geführt.

„Der Genderaspekt ist unumgehbar in Themenfeldern mit gesellschaftlicher Relevanz bzw. Konsumentenorientierung. Dennoch besteht vor allem im technischen Bereich Aufholbedarf bezüglich der Adressierung von genderrelevanten Fragestellungen."

„Wenn der Genderaspekt in Forschungsvorhaben eine Rolle spielt, dann kennt man das Förderprogramm auch."

Laut Einschätzung der interviewten FördernehmerInnen sind die FEMtech Forschungsprojekte dabei nicht geeignet, den Genderaspekt in bereits ausgestaltete Forschungsfragen beiläufig zu integrieren, um Mitnahmeeffekte generieren zu können. Dies lässt sich unter anderem auch auf die notwendige Genderkompetenz der AntragstellerInnen als Förderkriterium zurückführen, wobei von einer generellen Sensibilisierung im Bereich der Genderaspekte bei den FördernehmerInnen auszugehen ist.

\section{Allgemeine Beurteilung der Programmlinie}

Eine zusammenfassende Beurteilung der Programmlinie seitens der FördernehmerInnen ist zum jetzigen Zeitpunkt nur eingeschränkt möglich, da die Forschungsvorhaben der ersten Ausschreibung noch nicht abgeschlossen sind. Dennoch lässt sich die hohe Zufriedenheit der beteiligten Unternehmen und Forschungseinrichtungen, zumindest in den Projekten, die als Fallstudie erfasst wurden, erkennen. So ist etwa eine neuerliche Teilnahme an FEMtech Forschungsprojekte für die Befragten durchaus vorstellbar („sofort wieder, wenn ein geeignetes Projekt vorliegt"). Die Bearbeitung von Fragestellungen, welche in „normalen“, technischen Ausschreibungen nicht möglich gewesen wäre, begünstigt dabei den Aufbau zusätzlicher, organisationsinterner Genderkompetenzen. Darüber hinaus zeigt sich, dass diese Lerneffekte nicht ausschließlich auf das Projektkonsortium begrenzt sind, sondern etwa im Zuge von Beteiligungsprozessen schon während der Projektdurchführung auf die gesellschaftliche Ebene überschwappen können. 
Die FEMtech Forschungsprojekte als komplementäres Angebot zu den vorhandenen Förderangeboten mit genderrelevanten Fragestellungen werden von den befragten Unternehmen als notwendige Form der Unterstützung von Forschungsvorhaben angesehen. Daher sollte in Bezug auf die Förderung des Genderthemas eine Doppelstrategie gefahren werden, welche sowohl die genderzentrierte Bearbeitung als auch Gender als Querschnittsthema von FTI-Aktivitäten ermöglicht. Als mögliche Gefahr wird von Seiten der Befragten jedoch auch eine etwaige Substitution des Genderbewusstseins formuliert. Durch die Berücksichtigung genderrelevanter Themeninhalte in speziellen Förderlinien könnte die Bedeutung bzw. Einbeziehung in allgemeinen Projekten vernachlässigt werden.

Darüber hinaus verleiht die Programmlinie FEMtech Forschungsprojekte dem Genderaspekt im Bereich der technischen Forschungsfelder einen höheren Stellenwert. Der teilweise vernachlässigten Berücksichtigung geschlechterunterschiedlicher Bedürfnisse in den relevanten Themengebieten kann mit Hilfe der Signalfunktion der Programmlinie entgegengewirkt und die Know-how Intensität des Forschungsbereiches betont werden.

Angeregt wurde von den Fördernehmerlnnen hingegen, gemeinsame Meetings auch während des Projektverlaufes anzubieten ${ }^{17}$. Dadurch würde sich einerseits die fortlaufende Möglichkeit der Vernetzung ergeben, zum anderen könnten Einblicke in die unterschiedlichen Forschungstätigkeiten gewonnen und Ergebnisse aufgegriffen werden, die auch für die eigene Projektentwicklung relevant sein können.

„Die Auftaktveranstaltung bzw. das Netzwerktreffen zu Beginn der FTI-Aktivitäten war eine geeignete Maßnahme, um Projektinhalte und Vorgehensweisen darzustellen bzw. sich mit anderen Forschungsteams auszutauschen. Leider wurde dieser Informationsaustausch in weiterer Folge nicht mehr angeboten, weshalb Einblicke in Erfahrungen und Ergebnisse anderer Projekte nicht mehr möglich waren“.

17 Die teilnehmenden FördernehmerInnen hatten die Möglichkeit ihr Forschungsvorhaben kurz zu präsentieren bzw. die Projekte der weiteren TeilnehmerInnen kennenzulernen. 


\subsubsection{FEMtech Karriere}

FEMtech Karriere unterstützt strukturelle und nachhaltige Maßnahmen, die zur Chancengleichheit von Frauen und Männern in FTI Unternehmen und außeruniversitären Forschungseinrichtungen im naturwissenschaftlich-technischen Bereich führen.

Tabelle 4 Eckpunkte von FEMtech Karriere

\begin{tabular}{|c|c|}
\hline FEMtech Karriere & Kurzbeschreibung \\
\hline \multirow{2}{*}{ Ziele } & $\begin{array}{l}\text { Die Schaffung fairer Rahmenbedingungen für Frauen und Männer in } \\
\text { FTI-Unternehmen }\end{array}$ \\
\hline & $\begin{array}{l}\text { Die Erhöhung des Anteils von Frauen auf allen Hierarchieebenen } \\
\text { sowie ihrer Karrierechancen }\end{array}$ \\
\hline \multirow{2}{*}{ Zielgruppen } & $\begin{array}{l}\text { F\&E-Unternehmen und außeruniversitäre Forschungseinrichtungen } \\
\text { in Naturwissenschaft und Technik (Fördernehmerlnnen) }\end{array}$ \\
\hline & $\begin{array}{l}\text { Frauen und Männer in forschungs- und technologieintensiven Unter- } \\
\text { nehmen und außeruniversitären Forschungseinrichtungen }\end{array}$ \\
\hline \multirow[b]{2}{*}{ Inhalt } & $\begin{array}{l}\text { Förderung von strukturellen und nachhaltigen Maßnahmen, die zur } \\
\text { Chancengleichheit führen und Forscherinnen/Technikerinnen in der } \\
\text { Umsetzung ihrer beruflichen Ziele unterstützen }\end{array}$ \\
\hline & $\begin{array}{l}\text { Ein FEMtech Karriere Projekt folgt einem modularen Aufbau mit zwei } \\
\text { Pflichtmodulen (Aufbau Genderkompetenz und Projektmanagement) } \\
\text { und mehreren Wahlmodulen (Öffentlichkeitsarbeit, Personalma- } \\
\text { nagement, Work-Life-Balance und Karriereentwicklung/Men- } \\
\text { toring/Coaching). }\end{array}$ \\
\hline Einreichverfahren & Laufende Einreichung \\
\hline Laufzeit & Mindestens sechs Monate bis maximal zwei Jahre. \\
\hline Förderhöhe & $\begin{array}{l}\text { Max. } € 50.000 .- \text { (Förderquote } 50 \% \text { bis } 70 \% \text { je nach Größe der } \\
\text { einreichenden Organisation) }\end{array}$ \\
\hline
\end{tabular}

Durch FEMtech Karriere Projekte sollen u. a. die Genderkompetenz v. a. der Führungskräfte gesteigert werden, Leistungen von Frauen und Männern in FTI sichtbar gemacht werden, mehr Forscherinnen und Technikerinnen in Führungspositionen gelangen, eine gezielte Karriereentwicklung von Frauen betrieben werden sowie das Thema Chancengleichheit in Unternehmensprozesse und -strategien integriert und nachhaltig verankert werden.

Jedes FEMtech Karriere Projekt besteht aus zwei Pflichtmodulen (Aufbau Genderkompetenz und Projektmanagement) und mehreren Wahlmodulen (aus den Bereichen Öffentlichkeitsarbeit, Personalmanagement, Work-Life-Balance sowie Coaching/Mentoring und Karriereentwicklung bzw. frei wählbar), wobei ein vielfältiges Maßnahmenportfolio und die Kombination mehrerer Wahlmodule empfohlen wird, um eine nachhaltige Einbettung des Projekts in die Unternehmensstruktur zu gewährleisten. 
In den Ausschreibungsunterlagen werden die Module näher erläutert und auch anhand von Beispielen konkrete Maßnahmen illustriert. Diese Beispiele werden von den Interviewten als sehr hilfreich eingestuft.

Im Beobachtungszeitraum der gegenständlichen Evaluierung (2011 bis 2013) wurden insgesamt acht FEMtech Karriere Projekte beantragt und gefördert. Damit konnte die Zielgröße laut Programmdokument von 15 eingereichten bzw. 10 geförderten Projekten pro Jahr nicht annähernd erreicht werden. Die Projekte wurden von fünf Unternehmen (zwei Großunternehmen, drei KMU), zwei Kompetenzzentren und einer außeruniversitären Forschungseinrichtung aus Niederösterreich (ein Projekt), der Steiermark (drei Projekte) und Wien (vier Projekte) eingereicht. Die Hälfte der Projekte kam bis Ende 2013 zu einem Abschluss, drei Projekte enden im ersten Halbjahr 2014. Ein Projekt startete erst im Herbst 2013 und läuft bis 2015.

\section{Motivation zur Einreichung}

Bei den Fördernehmerlnnen handelt es sich Großteils um Organisationen, die sich mit dem Genderthema bereits befasst haben bzw. um Organisationen, deren Personalverantwortliche für das Thema sensibilisiert wurden bzw. die sich z. B. einer Diversity-Strategie verschrieben haben. So finden sich auch zwei Fördernehmerlnnen, in deren Leistungsportfolio der Schwerpunkt Gender zu finden ist, und die nun ihre eigenen Strukturen entsprechend adaptieren möchten.

Allen Fördernehmerlnnen scheint grundsätzlich die Wichtigkeit der Sicherung von Fachkräften für den Erfolg ihres Unternehmens bewusst. Folgende Motive zur Konzipierung und Durchführung eines FEMtech Karriere Projekts lassen sich unterscheiden:

- Die Organisation will Gender Mainstreaming-Maßnahmen implementieren. Meist geschieht dies vor dem Hintergrund, dass Frauen in den Unternehmen stark unterrepräsentiert sind und der Frauenanteil gesteigert werden soll, um dem Fachkräftemangel zu begegnen und um die Vorteile gemischter Teams nützen zu können.

- Die Organisation hat einen hohen Frauenanteil und will die Work-Life Balance verbessern bzw. geeignete Modelle erarbeiten, um v. a. Frauen Karriereperspektiven bieten zu können.

- Die Organisation führte schon einmal ein FEMtech Karriere Projekt durch und will die gesetzten Maßnahmen fortsetzen, erweitern bzw. nachhaltig verankern.

- Das FEMtech Karriere Projekt soll dabei unterstützen, konkrete Personalentwicklungsziele zu erreichen. Meist geht dies mit anstehenden Umstrukturierungsmaßnahmen einher. 
Der Einsicht über die Notwendigkeit von Personalentwicklungsmaßnahmen im Allgemeinen und Maßnahmen zur Erhöhung der Chancengleichheit im Besonderen stehen aber meist die fehlenden Ressourcen gegenüber. Erst durch die Förderung im Rahmen von FEMtech Karriere scheint die Möglichkeit der Durchführung solcher Maßnahmen gegeben, v. a. wenn diese umfassend und breit verankert werden sollen. Zumindest jene Organisationen, die im Rahmen der gegenständlichen Evaluierung in Form von Fallstudien (siehe Anhang) näher untersucht wurden, berichten von sehr hoher Additionalität.

„In diesem Rahmen wäre das nicht möglich gewesen. Wir sind ein sehr kleines Unternehmen, wir hätten natürlich auch die finanziellen Mittel in dem Maße nicht aufbringen können."

"Was nicht auf Projekte gebucht werden kann, ist ein Problem."

„Ich hätte sämtliche Maßnahmen nicht umsetzen können, weil ich nicht das Budget bekommen hätte."

\section{Projektinhalte}

Das Pflichtmodul „Aufbau von Genderkompetenz" sieht verpflichtend das Training der Führungskräfte und Schlüsselpersonen vor. In den meisten Fällen geschieht dies zum einen über (externe) Schulungen der ProjektleiterInnen oder von in der Folge als Genderbeauftragte installierten Personen. Zum anderen werden interne Workshops meist unter der Leitung externer GenderexpertInnen durchgeführt. TeilnehmerInnen sind immer die (männlichen) Führungskräfte des Unternehmens (meist mehrere Führungsebenen wie Geschäftsleitung, Abteilungsleitung, Projektleitung, Senior Researcher), oftmals auch Schlüsselkräfte (z. B. HRMitarbeiterlnnen) und teilweise auch alle MitarbeiterInnen (v. a. bei Kleinunternehmen). Zu diesem Modul zählt aber auch die Erhebung des Status quo in der Organisation in Bezug auf die Repräsentanz von Frauen auf den verschiedenen Hierarchiestufen.

Das zweite Pflichtmodul „Projektmanagement" umfasst die effiziente Koordination und Abwicklung des Projekts, die Umsetzung der Maßnahmen laut Plan sowie die aktive Einbindung von Führungskräften in das Projekt ebenso wie ein gemischtes Projektteam.

Die Verankerung gendersensibler Kommunikation steht beim Modul „Öffentlichkeitsarbeit" im Vordergrund. Sieben der acht geförderten Projekte inkludieren dieses Modul. Zu unterscheiden sind die interne und die externe Kommunikation. Zur Verbreitung der Projektinhalte nach innen wurden z. B. Informationsveranstaltungen gehalten oder die Intranet-Seite umgestaltet, um über die Aktivitäten im Projekt zu berichten. Extern stand in vielen Fällen die Adaptierung der Homepage im Vordergrund. Die Homepage wird nun auch vielfach genutzt, um Technikerinnen oder weibliche Führungskräfte als Role Models zu präsentieren. 
Diese werden in Folge des FEMtech Karriere Projektes auch bei der Entsendung zu Veranstaltungen verstärkt berücksichtigt. Zudem wurden Informationsmaterialien und Geschäftsberichte etc. bis hin zu einem Messestand nach Gendergesichtspunkten durchleuchtet und neu gestaltet, um verstärkt Frauen für eine Karriere als Forscherin ansprechen zu können.

Das Unternehmen als attraktiven Arbeitgeber zu positionieren steht im Mittelpunkt des Moduls „Personalmanagement". Im Bereich Recruiting reichen die Maßnahmen im Rahmen der geförderten FEMtech Karriere Projekte über die Durchleuchtung der Recruitingprozesse und die Abhaltung von Führungskräfte-Workshops zum Thema "gendersensitives Recruiting“, die Erarbeitung von Aufgaben- und Kompetenzprofilen, die Intensivierung von Bildungspartnerschaften mit Bildungseinrichtungen bis zur Neugestaltung des Prozesses bei der Einführung neuer MitarbeiterInnen oder die Identifizierung von Role Models. Einige Maßnahmen zielten auf die Erarbeitung neuer gendersensitiver Personalentwicklungsstrategien.

Das Modul "Work-Life Balance“ hat zum Ziel, die individuelle Lebensplanung der MitarbeiterInnen mit den betrieblichen Anforderungen in Einklang zu bringen. Im Mittelpunkt der Projekte standen die Erarbeitung flexibler Arbeitszeitmodelle, die proaktive Gestaltung von Karenz und Wiedereinstieg und die Förderung der Väterkarenz. Im Zusammenhang mit flexiblen Arbeitszeiten wurden z. B. Modelle erarbeitet, wie der Kommunikationsstand der Mitarbeiterlnnen an einem Projekt trotz unterschiedlicher Anwesenheitszeiten optimiert werden kann. Einige Projekte beschäftigten sich auch mit den Themen Burn-out Prophylaxe und Gesundheitsförderung.

Im Modul „Coaching/Mentoring“ sollen Technikerinnen und Forscherinnen in ihrer Karriereentwicklung unterstützt werden. Die meisten Organisationen bieten hier den Mitarbeiterinnen, die als potentielle Führungskräfte identifiziert werden, Coaching und Mentoring zur Reflexion der (zukünftigen) Führungsrolle und der Stärkung der Führungskompetenzen. Wichtiges Thema der Coachings ist meist auch der Wechsel der Rolle von der Kollegin zur Vorgesetzten. Meist steht daher das Coaching in direktem Zusammenhang mit einem Karrieresprung; dementsprechend werden die Module "Coaching/Mentoring“ und „Karriereentwicklung" meist in Kombination umgesetzt.

Die Stärkung der Führungskompetenzen von Technikerinnen und Forscherinnen und die Sichtbarmachung von Frauen in Führungspositionen stehen im Mittelpunkt des Moduls „Karriereentwicklung“. Dieses Modul nützen einige Fördernehmerlnnen, um gezielt aus der Belegschaft Frauen für Führungspositionen zu identifizieren und mit einer Führungsrolle zu betrauen. Dies geht $z$. B. in Organisationen mit sehr flacher Hierarchie mit der Schaffung einer Führungsebene einher, die zwischen Geschäftsführung und Mitarbeiterlnnen eingezogen und durch die Änderung des Organigramms sichtbar wird. 
Einige FördernehmerInnen nützen die Möglichkeit, Wahlmodule zu definieren, z. B. im Bereich Talentemanagement oder Gehaltsmanagement oder für die Ausarbeitung eines Verhaltenskodex. Eine Organisation hat sich zum Ziel gesetzt, mit im Genderbereich aktiven Forschungsunternehmen ein Netzwerk in der Region zu etablieren.

\section{Ergebnisse und Wirkungen}

Die in den Fallstudien untersuchten Organisationen zeigen sich begeistert vom der Resonanz, die ihre FEMtech Karriere Projekte in ihren Unternehmen ausgelöst haben. Sie berichten von einer deutlich höheren Sensibilität der Führungskräfte der Genderthematik gegenüber. Neben diesen atmosphärischen Veränderungen haben die Fördernehmerlnnen aber auch in Zahlen nachweisbare Ergebnisse vorzuweisen, wie etwa eine Erhöhung des Anteils weiblicher TechnikerInnen oder mehr Bewerberinnen. Dies ist auch auf die Änderung in Recruitingprozessen und der Selbstdarstellung der Unternehmen sowie der verstärkten Sichtbarmachung von Frauen als Forscherinnen und Technikerinnen zurückzuführen. Frauen wurden im Rahmen der FEMtech Karriere Projekte in Führungspositionen befördert bzw. verstärkt in ein Führungskräfteprogramm eingebunden sowie in ihrem Selbstverständnis als Technikerinnen und als Führungspersönlichkeiten gestärkt.

„....weil wirklich was bewirkt wird. Da kommen Frauen zu uns an den Messestand mit dem Folder in der Hand und sagen, sie finden das so toll, sie finden sich so angesprochen von uns. Da haben wir direktes Feedback."

„Wir haben Beförderungen schon im Unternehmen gehabt. Wir haben seit Sommer die erste Gruppenleiterin, wäre vielleicht auch nicht so leicht gegangen ohne das Projekt."

„Da bewegt sich schon was."

Auch wurden flexible Arbeitszeitmodelle eingeführt. Dass Gendermaßnahmen nicht nur auf Frauen abzielen, sondern das gesamte Arbeitsklima verändern und beiden Geschlechtern zugute kommen können, zeigt z. B. eine stärkere Inanspruchnahme der Väterkarenz in zwei untersuchten Organisationen.

Obwohl viele Ziele in den Projekten erreicht wurden, betonen alle Projektverantwortlichen, dass umfassende Veränderungen und eine nachhaltige Verankerung von Chancengleichheit Zeit brauchen und nur langfristig Platz greifen können. Zudem setzten einige Organisationen auch auf anderen Maßnahmen, die das FEMtech Karriere Projekt unterstützen und ergänzen können. Drei der FördernehmerInnen bietet auch FEMtech Praktika für Studentinnen an, ein befragtes Unternehmen auch Schülerlnnenpraktika und in einem Fall ist die Organisation auch in einem Talente regional Projekt involviert. 
„Das ist nicht einfach mit dem Endbericht abgeschlossen. Wir möchten dieses Thema weiterbearbeiten, weil wir gesehen haben, dass das sehr viel bringt, nicht nur den Personen, den Mitarbeitern, sondern auch dem Unternehmen."

„Es geht einfach nicht von heut auf morgen, es kann nicht sein, dass man von heut auf morgen auf einmal doppelt so viele Frauen im Unternehmen hat, so schnell geht es nicht, aber es bewegt auf jeden Fall was auf mittel und lange Frist."

\section{Allgemeine Beurteilung der Programmlinie}

Obwohl nur sehr wenige FEMtech Karriere Projekte im Beobachtungszeitraum eingereicht und gefördert wurden und damit der Zielwert nicht erreicht werden konnte, scheint die Programmlinie bei den AntragstellerInnen hohe Akzeptanz zu genießen. Alle drei Organisationen, in denen Fallstudien durchgeführt wurden, halten FEMtech Karriere „für eine sehr gute Sache“. Die Förderung verleiht dem Thema Wichtigkeit und liefert den HR-Verantwortlichen gegenüber der Geschäftsführung schlagende Argumente, ein solches Personalentwicklungsprojekt durchzuführen bzw. stellt oftmals die einzige Möglichkeit dar, sich umfassend mit dem Thema Chancengleichheit zu befassen.

Die positive Beurteilung der Programmlinie ist auch zum einen daraus ersichtlich, dass alle befragten Organisationen diese weiterempfehlen würden und dies auch innerhalb ihrer Netzwerke bereits tun und zum anderen, dass sie sich sehr gut vorstellen können, wieder bei FEMtech Karriere mit anderen Themenschwerpunkten einzureichen.

Den Unternehmen/Forschungseinrichtungen ist aber bewusst geworden, dass trotz der zahlreichen konkreten Ergebnisse die Nachhaltigkeit der Verankerung von Chancengleichheit nur mittel- bis langfristig gelingen kann. Diese langfristige Perspektive könnte ein Grund für die Zurückhaltung bei den Einreichungen sein, weil die unmittelbaren Kosten (da keine 100 \%-Förderung) einem zukünftigen Nutzen gegenüberstehen. Zudem wird deutlich, dass nur Organisationen eingereicht haben, die für das Thema schon zu einem gewissen Grad sensibilisiert waren bzw. in dem einzelne Personen von der Wichtigkeit des Themas überzeugt und auf die Agenda gesetzt haben. Eine Mobilisierung von „unbedarften“ FTI-Unternehmen und Forschungseinrichtungen dürfte unter den derzeitigen wirtschaftlichen Rahmenbedingungen und unter den derzeitigen Förderbedingungen nicht gelingen. 


\subsubsection{FEMtech Praktika für Studentinnen}

Das Förderangebot FEMtech Praktika für Studentinnen zielt auf die Gewinnung von weiblichen Nachwuchskräften im Themenbereich naturwissenschaftlich-technischer Forschungsfelder ab. Durch die aktive Mitarbeit der Studentinnen an Forschungsprojekten in Unternehmen und außeruniversitären Forschungseinrichtungen werden Einblicke in die Berufspraxis und Unterstützung beim Einstieg in eine Forschungskarriere ermöglicht.

Tabelle 5 Eckpunkte von FEMtech Praktika

\begin{tabular}{|c|c|}
\hline FEMtech Praktika & Kurzbeschreibung \\
\hline Ziele & $\begin{array}{l}\text { Gewinnung von Studentinnen für Karrieren in der angewandten For- } \\
\text { schung im naturwissenschaftlich-technischen FTI-Bereich. }\end{array}$ \\
\hline \multirow[b]{2}{*}{ Zielgruppen } & $\begin{array}{l}\text { Unternehmen und außeruniversitäre Forschungseinrichtungen in } \\
\text { Naturwissenschaft und Technik (Fördernehmerlnnen). }\end{array}$ \\
\hline & $\begin{array}{l}\text { Weibliche Nachwuchsforscherinnen die an einer österreichischen, } \\
\text { naturwissenschaftlichen oder technischen Universität bzw. Fachhoch- } \\
\text { schule inskribiert sind. }\end{array}$ \\
\hline Inhalt & $\begin{array}{l}\text { Förderung von hochwertigen Praktika für forschungsinteressierte } \\
\text { Studentinnen ermöglicht Einblicke in die Berufspraxis und das Knüp- } \\
\text { fen wertvoller Kontakte. }\end{array}$ \\
\hline Einreichverfahren & $\begin{array}{l}\text { Laufende Einreichung nach Ausschreibung, bei Mittelausschöpfung } \\
\text { vorzeitiges Schließen der Ausschreibung. Ausschreibung } 2011 \text { Okto- } \\
\text { ber bis Juni, Ausschreibung } 2012 \text { September bis Juni. Nächste Aus- } \\
\text { schreibung Mai } 2014 \text {. }\end{array}$ \\
\hline Laufzeit & $\begin{array}{l}\text { Praktikumsdauer ein bis sechs Monate (Beschäftigungsausmaß min- } \\
\text { destens } 28,5 \text { Wochenstunden). }\end{array}$ \\
\hline Förderhöhe & $\begin{array}{l}\text { Die geplante Förderhöhe ist abhängig von der Praktikumsdauer und } \\
\text { beträgt zwischen } € 2.100 \text {.- (ein Monat) und } € 10.600 \text {.- (sechs Monate). } \\
\text { Die im Mai } 2014 \text { startende Ausschreibung sieht etwas niedrigere Be- } \\
\text { träge vor. }\end{array}$ \\
\hline
\end{tabular}

Im Untersuchungszeitraum hat es zwei Ausschreibungen für FEMtech Praktika für Studentinnen gegeben (Oktober 2011 bis Mai 2012, sowie September 2012 bis Mai 2013), welche trotz zwischenzeitlicher Mittelaufstockung auf Grund vorzeitiger Budgetausschöpfung frühzeitig geschlossen werden mussten. Letztendlich wurden im Zuge der ersten Ausschreibung 234 von 262 eingereichten Praktikumsstellen gefördert (89\%) und im Zuge der zweiten Ausschreibung 490 von 499 (98\%). Dass sich die Zahl der eingereichten bzw. geförderten Praktikumsplätze gegenüber der ersten Ausschreibung mehr als verdoppeln konnte, ist auf die ebenfalls deutlich angehobene Gesamtbudgetsumme der zweiten Ausschreibung zurückzuführen. Diese beinhaltete unter anderem die Mittel der für Herbst 2013 geplanten dritten Ausschreibung, welche auf Grund der Mittelvorziehung entfallen ist. 
Die Bereitstellung und Aufstockung der Fördermittel ermöglichte das Erreichen der angestrebten Zielgröße von 200 bis 300 Praktika pro Ausschreibung. ${ }^{18}$

Die Anbieterlnnen von Praktikumsplätzen können sowohl Unternehmen als auch außeruniversitäre Forschungseinrichtungen im naturwissenschaftlich-technischen F\&E-Bereich sein. Die erste und zweite Ausschreibung wurde diesbezüglich von den Forschungseinrichtungen dominiert (2011: 60 \%, 2012: 58 \%). Die geschaffenen Praktika im Bereich der Unternehmen konzentrieren sich wiederum hauptsächlich in den KMU (29\%), trotz leichter Zugewinne der Großunternehmen bei der zweiten Ausschreibung (2011: $11 \%$, 2012: $13 \%$ ).

Auf regionaler Ebene zeigt sich ein leichtes Ost-West Gefälle zwischen den begünstigten Fördernehmerlnnen. Sowohl 2011 (58 \%) als auch 2012 (61\%) dominierten die Bundesländer Wien und Niederösterreich das Praktikumsangebot. Ebenfalls Anteile im zweistelligen Prozentbereich weisen die steirischen FTIAkteurlnnen auf (2012: $17 \%$ ), während die übrigen Bundesländer in Summe rd. ein Fünftel der Praktikantinnen beschäftigt haben (2011: 18 \%, 2012: $21 \%$ ). Gemäß Zielsetzung von FEMtech Praktika für Studentinnen sind 9 von 10 Praktikumsplätzen in den Themenfelder Technik (2011, 2012: 34 \%) und Naturwissenschaften (2011: 59 \%, 2012: 56 \%) angesiedelt. Praktika, die nicht in diese Wissenschaftsdisziplinen fallen, entstammen den Bereichen Humanmedizin sowie Land- und Forstwirtschaft.

Die Praktikumsdauer kann je nach Bedarf zwischen einem und sechs Monaten liegen. Es zeigt sich, dass in beiden Ausschreibungsjahren sechsmonatige Praktikumsstellen am häufigsten vergeben worden sind (siehe Grafik 19 im Anhang). Kurzfristige „Ferialpraktika“ mit einer Dauer von ein, zwei oder drei Monaten wurden jeweils von rd. $15 \%$ der Studentinnen in Anspruch genommen. Die durchschnittliche Praktikumsdauer im Jahr 2011 betrug vier Monate, 2012 lag der Mittelwert bei 4,2 Monaten. Betrachtet man die Verteilung der Praktikantinnen hinsichtlich ihres Studienfortschritts, so zeigt sich, dass die FEMtech Praktika für Studentinnen in sämtlichen Phasen des Studiums in Anspruch genommen werden. Der deutlich überwiegende Teil der Studierenden befindet sich in den Semestern zwei bis acht.

Dass die Programmlinie FEMtech Praktika für Studentinnen ein direktes Bindeglied zwischen akademischer Ausbildung, Berufseinstieg und Karriereentwicklung darstellt, zeigen die Angaben der Studentinnen zu den praktikumsübergreifenden Anstellungen: Für $20 \%$ (2011) bzw. $18 \%(2012)^{19}$ der im Zuge der StudentinnenFeedbacks befragten Teilnehmerinnen führte das Praktikum nach Abschluss zu einer weiterführenden Anstellung. 
Explizite Angaben zu den Weiteranstellungen wurden der FFG für insgesamt 43 Praktika (Stand: Juni 2013) gemeldet. 28 davon sind in unbefristete Dienstverhältnis überführt wurden, weitere 11 Praktikumsplätze wurden nach Auslaufen der Förderung verlängert und vier Studentinnen wurde eine Anstellung zu einem späteren Zeitpunkt angeboten ${ }^{20}$.

\section{Feedback Studentinnen}

Drei Viertel der FEMtech Praktika für Studentinnen Teilnehmerinnen besuchten zum Zeitpunkt des Praktikums eine Universität, die übrigen rd. $25 \%$ eine Fachhochschule. Diese Ausbildungsstätten waren auch in rd. $22 \%$ aller Fälle für die Bekanntmachung bzw. Bewerbung des Förderangebots bei den Studentinnen verantwortlich. Nur die Unternehmen und Forschungseinrichtungen selber haben häufiger auf die Möglichkeit des Praktikums hingewiesen (rd. $55 \%$ ). Weitere rd. $20 \%$ der Praktikantinnen wurden von Ihrem Umfeld auf die Möglichkeit des Forschungspraktikums aufmerksam gemacht.

Fast $90 \%$ der Studentinnen beider Ausschreibungsrunden haben angegeben, dass innen das Praktikum insgesamt sehr gut oder gut gefallen hat (2011: $89 \%$, 2012: $87 \%$ ). Hingegen haben jeweils nur rd. $3 \%$ das Praktikum mit „nicht genügend" beurteilt. Überwiegend wurden die FEMtech Praktika für Studentinnen aus Gründen der Berufserfahrung absolviert (2011: 69 \%, 2012: 74 \%). 40 \% der Studentinnen haben das Einkommen als einen weiteren wesentlichen Aspekt der Praktikumsteilnahme genannt und rd. $25 \%$ sahen eine Möglichkeit zur Abhandlung ihrer Diplomarbeit.

Überwiegend sehr gut beurteilten die Studentinnen die Betreuung seitens der PraktikumsgeberInnen (Durchschnittsbenotung 2011 und 2012 jeweils: 1,4) sowie die Zusammenarbeit in Bezug auf die Forschungstätigkeiten (2011: 1,5 2012: 1,6). Darüber hinaus beurteilten $90 \%$ der Praktikantinnen beider Ausschreibungsrunden das Anspruchsniveau ihrer Tätigkeiten als sehr anspruchsvoll bis anspruchsvoll. Rd. die Hälfte der Teilnehmerinnen schätzt des Weiteren die FEMtech Praktika für Studentinnen als anspruchsvoller ein als nicht geförderte Praktika. Die Vereinbarkeit von Beruf und Studium wurde ebenfalls als überwiegend sehr gut empfunden (2011: 1,7 2012: 1,8), während die Sichtbarkeit von karriereunterstützenden Maßnahmen im Unternehmen bzw. der Forschungseinrichtung insgesamt gut wahrgenommen wurde (2011 und 2012 jeweils 2,2).

Deutlich positive Wirkungen hatte die Programmlinie FEMtech Praktika für Studentinnen auch auf das Interesse der Teilnehmerinnen im naturwissenschaftlichtechnischen Forschungsbereich zu arbeiten. 75 \% (2011) bzw. 69 \% (2012) der Studentinnen haben angegeben, dass ihr Interesse stark bzw. sehr stark gestiegen ist, bei weiteren $21 \%$ bzw. $28 \%$ ist das Interesse unverändert geblieben. Diese

20 Quelle: FFG Monitoringdaten 
Entwicklung wurde unter anderem durch die als sehr gut bewertete Möglichkeit (die Durchschnittsbenotung liegt im Ausschreibungsjahr 2011 bei 1,5 bzw. im Jahr 2012 bei 1,7) begünstigt, Einblicke in die Welt der Forschung zu gewinnen bzw. sich Fachwissen anzueignen (2011: 1,5 2012: 1,4) und drückt sich auch in einer positiven Einstellung gegenüber einer neuerlichen Teilnahme an dem Förderprogramm aus (2011: 2,3 2012: 1,6). Dementsprechend haben nahezu $100 \%$ der Studentinnen angegeben, dass sie FEMtech Praktika für Studentinnen auch weiterempfehlen werden.

„Das Praktikum hat mir ermöglicht, einen besseren Einblick in den wissenschaftlichen Alltag zu bekommen, sowie mir neue Analysetechniken anzueignen. Darüber hinaus konnte ich sehr gute Netzwerke aufbauen und freu mich auf die künftige Zusammenarbeit."

\section{Feedback Unternehmen und Forschungseinrichtungen ${ }^{21}$}

Die Förderungen im Rahmen von FEMtech Praktika für Studentinnen werden von den Unternehmen und Forschungseinrichtungen allgemein als effizient gestaltetes Instrument gesehen. Nahezu $100 \%$ der geförderten Unternehmen und Forschungseinrichtungen haben das Verhältnis zwischen Formalaufwand und Förderungsleistung zumindest als ausgewogen empfunden. Ebenso übersteigt der Nutzen der Einbindung von Studentinnen in die Forschungsaktivitäten der FördernehmerInnen den damit verbundenen Aufwand in nahezu allen Fällen. Dabei profitieren nach eigenen Angaben jeweils zwei Drittel der teilnehmenden Unternehmen und Forschungseinrichtungen unmittelbar oder langfristig.

„Eine der wenigen Möglichkeiten zur Förderung technischer Entwicklungen im Rahmen der Grundlagenforschung in kleinem Rahmen."

„Für unsere Organisation haben die guten Leistungen der Studentin einen echten Mehrwert gebracht. Die administrativen Hürden sind gering und der Dokumentationsaufwand auf ein Minimum reduziert."

Es ermöglichte uns, überhaupt ein Praktikum anzubieten und hat entscheidend dazu beigetragen, passende, künftige Mitarbeiter zu gewinnen."

Aus der untenstehenden Grafik wird ersichtlich, dass der von beiden Seiten (FördernehmerInnen und Studentinnen) erzielte Nutzen durch die Förderung im Rahmen von FEMtech Praktika für Studentinnen überhaupt erst ermöglicht worden ist. In beiden Ausschreibungen lag der Anteil der Fördernehmerlnnen, die Praktikumsstellen auch ohne Förderung in unverändertem Ausmaß angeboten hätten, im niedrigen einstelligen Prozentbereich. Mehr als die Hälfte der Befragten gaben des Quelle: Feedback der Förderungsnehmerlnnen FEMtech Praktika für Studentinnen N=223 (2011) bzw. 345 (2012) 
Weiteren an, dass innen die Möglichkeit gegeben wurde, mehr Studentinnen zu beschäftigten, als ursprünglich geplant. In $38 \%$ bis $48 \%$ der Fälle wurde das Anbieten von Praktikumsstellen überhaupt erst durch die Förderung ermöglicht (siehe Grafik 9). Anders ausgedrückt hätten rd. 112 bzw. 186 Forschungspraktika ohne FEMtech Praktika für Studentinnen nicht angeboten werden können. Damit weist diese Programmlinien eine vergleichsweise hohe Additionalität auf.

\section{Grafik 9 Additionalität FEMtech Praktika für Studentinnen}

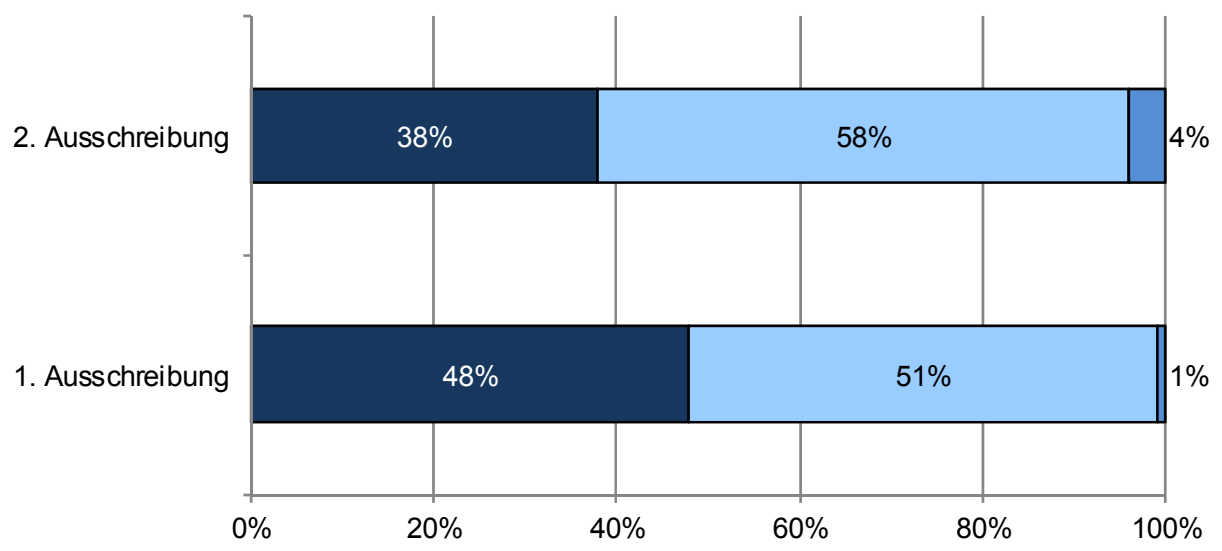

घkonnten Praktika anbieten $\square$ konnten mehr Studentinnen anstellen $\quad$ akein Einfluss

Quelle: Feedback FörderungsnehmerInnen, n=223 (2011), 345 (2012)

Die positiven Erfahrungen sowohl mit der Abwicklung und Durchführung der Programmlinie, als auch der beidseitige Nutzen für Studentinnen und Fördernehmerlnnen bewirkten, dass eine überwiegende Mehrheit der Unternehmen und Forschungseinrichtungen sich auch an künftigen Ausschreibungen beteiligen möchte (2011: $73 \%$, 2012: $80 \%$ ). Weitere $22 \%$ bzw. $17 \%$ gehen von einer wahrscheinlichen weiteren Inanspruchnahme der FEMtech Praktika für Studentinnen aus. 


\subsubsection{FEMtech Dissertationen/Dissertationen in den Themen des BMVIT}

Im Rahmen der Programmlinie FEMtech Dissertationen/Dissertationen in den Themen des BMVIT werden Dissertationsprojekte von StudentInnen gefördert, die für die Dauer des Dissertationsprojekts in einem Unternehmen bzw. einer außeruniversitären Forschungseinrichtung angestellt werden. Gegenstand der vorliegenden Evaluierung ist die erste Ausschreibung FEMtech Dissertationen im Thema Produktion, im Rahmen derer ausschließlich Dissertationen von weiblichen Studierenden gefördert wurden.

Tabelle 6 Eckpunkte von FEMtech Dissertationen/Dissertationen in den Themen des BMVIT

\begin{tabular}{|l|l|}
\hline $\begin{array}{l}\text { FEMtech } \\
\text { Dissertationen }\end{array}$ & Kurzbeschreibung \\
\hline Ziele & $\begin{array}{l}\text { Unterstützung von Dissertationsprojekten in Unternehmen und } \\
\text { Forschungseinrichtungen. }\end{array}$ \\
\hline Zielgruppen & $\begin{array}{l}\text { Unternehmen, Kompetenzzentren und Forschungseinrichtungen } \\
\text { (Fördernehmerlnnen). } \\
\text { Dissertationsprojekte von weiblichen und männlichen Nachwuchs- } \\
\text { wissenschafterlnnen (im Rahmen der ersten Ausschreibung wur- } \\
\text { den nur weibliche Studierende gefördert) }\end{array}$ \\
\hline Inhalt & $\begin{array}{l}\text { Förderung von Dissertationsvorhaben zur aktiven Unterstützung } \\
\text { und Integration der Dissertantlnnen in das Unternehmen/ For- } \\
\text { schungseinrichtung. Bei den Dissertationen in den Themen des } \\
\text { BMVIT sind zumindest 50 \% der Dissertationsprojekte für Disser- } \\
\text { tantinnen vorgesehen (bei der ersten Ausschreibung wurden aus- } \\
\text { schließlich Dissertantinnen gefördert). }\end{array}$ \\
\hline Einreichverfahren & $\begin{array}{l}\text { Laufende Einreichung nach Ausschreibung (Mai bis Oktober 2012, } \\
2013) . \text { Nächster geplanter Ausschreibungstermin Mai 2014 (erfolgt } \\
\text { nicht mehr im Rahmen von Talente) }\end{array}$ \\
\hline Laufzeit & \begin{tabular}{l} 
Mindestens zwei Jahre bis maximal drei Jahre. \\
\hline Förderhöhe
\end{tabular} \\
Bis zu $€ 100.000 .-$ pro Dissertationsprojekt \\
\hline
\end{tabular}

Insgesamt hat es bisher zwei Ausschreibungen für die Programmlinie FEMtech Dissertationen/Dissertationen in den Themen des BMVIT im Rahmen des Förderschwerpunkts Talente gegeben. Im Zuge der ersten Ausschreibung im Jahr 2012 wurden insgesamt 10 Projektanträge eingereicht, von denen fünf gefördert wurden, drei von außeruniversitären Forschungseinrichtungen und zwei von Unternehmen (zwei dieser Einrichtungen haben ihren Sitz in Kärnten, zwei in Niederösterreich und eine in Wien). Im Rahmen der zweiten Ausschreibung im Jahr 2013 wurden insgesamt 13 Projektanträge eingereicht, die alle gefördert werden konnten. Da sich die Projekte der zweiten Ausschreibungsrunde zum gegenwärtigen Zeitpunkt noch in der Startphase befinden, konnten diese nicht in die gegenständliche Evalu- 
ierung einbezogen werden. Damit beziehen sich die erzielten Erkenntnisse ausschließlich auf die fünf im Rahmen der ersten Ausschreibung geförderten Projekte.

Die erste Ausschreibung von FEMtech Dissertationen im Thema Produktion ${ }^{22}$, die ausschließlich Dissertationen von weiblichen Studierenden förderte ${ }^{23}$, zielte darauf ab, die Karrierechancen von Frauen in Naturwissenschaft bzw. Technik zu verbessern, den erforderlichen Nachwuchs an Forscherinnen im Thema Produktion zu unterstützen sowie Forscherinnen an österreichische Unternehmen in der Forschung und Entwicklung bzw. an außeruniversitäre Forschungseinrichtungen heranzuführen und aufzubauen. Darüber hinaus sollte den Dissertantinnen eine Einstiegsmöglichkeit in das Berufsleben der angewandten Forschung - konkret im Bereich Produktion und Material - geboten werden.

Die im Folgenden dargestellten Ergebnisse der Evaluierung in Bezug auf die Programlinie FEMtech Dissertationen basieren auf telefonischen Interviews mit den Verantwortlichen der fünf geförderten Projekte aus der ersten Ausschreibungsrunde. Dabei ist zunächst festzuhalten, dass die Programmlinie FEMtech Dissertationen in Hinblick auf die verfolgten Zielsetzungen ein sehr hohes Potenzial aufweist und zumindest im Rahmen der fünf geförderten Projekte der ersten Ausschreibung bereits einen nachhaltigen Beitrag zur Höherqualifizierung von Frauen im Bereich Naturwissenschaft und Technik bzw. deren Heranführung an das Berufsleben leisten konnte. Durch FEMtech Dissertationen konnten Studentinnen zum Verfassen einer Dissertation motiviert werden, was sie - zumindest in einigen der untersuchten Fälle - ohne das FEMtech Dissertationen Projekt nicht getan hätten. Darüber hinaus verfügen einige Dissertantinnen bereits über eine Vollzeitanstellung (z.T. mit unbefristetem Vertrag) bei den sie betreuenden Unternehmen bzw. Forschungseinrichtungen und können damit bereits einen erfolgreichen Berufseinstieg in der angewandten Forschung verzeichnen.

\section{Motivation zur Projektteilnahme und Erfahrungshintergrund}

Die Fördernehmerlnnen der ersten Ausschreibung von FEMtech Dissertation haben überwiegend über die Homepage der FFG von der Programmlinie erfahren. Ausgangspunkt war in den meisten Fällen das Vorhandensein einer Projektidee bzw. eines Forschungsthemas, für das dann gezielt nach entsprechenden Fördermöglichkeiten gesucht wurde. Dabei war für die Unternehmen und Forschungseinrichtungen eher ausschlaggebend, dass die Programmlinie inhaltlich/thematisch inrem Vorhaben entsprochen hat, als die Tatsache, dass es sich hier um ein Programm zur Förderung von Frauen in Naturwissenschaft und Technik handelt.

22 Die zweite Ausschreibung erfolgte im Thema Mobilität der Zukunft.

23 In der zweiten Ausschreibung wurde die Programmlinie auch für männliche Nachwuchswissenschaftlerlnnen geöffnet, wobei mindestens $50 \%$ der Dissertationsprojekte von Dissertantinnen durchzuführen sind. 
Die bisherigen Erfahrungen mit frauenfördernden Maßnahmen sind bei den Projektnehmerlnnen sehr heterogen. Während einige gar keine spezifischen Aktivitäten zur Förderung von Frauen innerhalb ihres Unternehmens bzw. ihrer Forschungseinrichtung umsetzen bzw. aufweisen, kommt dem Thema bei anderen eine recht hohe Bedeutung zu, die durch ein breites Bündel an Gleichstellungsaktivitäten sowie entsprechende Auszeichnungen unterstrichen wird (eine im Rahmen von FEMtech Dissertationen geförderte Forschungseinrichtung hat den Staatspreis für Chancengleichheit bekommen; einem weiteren Unternehmen wurde vom damaligen Wirtschaftsministerium das Vollzertifikat für „berufundfamilie“ verliehen).

\section{Verbesserung der Karrierechancen von Frauen}

FEMtech Dissertationen trägt eindeutig zur Verbesserung der Karrierechancen von Frauen in Naturwissenschaft und Technik bei und erfüllt damit eine seiner wesentlichen Zielsetzungen. Durch die Programmlinie gelingt es, Frauen bei der Höherqualifizierung zu unterstützen bzw. dazu zu motivieren. In zumindest zwei der untersuchten Fälle war FEMtech Dissertationen sogar ausschlaggebend dafür, dass sich die Dissertantinnen überhaupt dazu entschlossen haben zu promovieren.

„Die Dame wollte nicht unbedingt dissertieren, sie wusste nicht, ob sie es schaffen würde oder nicht. Hat es sich aber überlegt und war dann bereit, es zu probieren und bei FEMtech Dissertationen mitzumachen."

„Die Dissertantin wollte nicht unbedingt promovieren, dann hat sie das Projekt aber sehr interessiert und deshalb hat sie sich dann entschlossen doch eine Diss zu schreiben. Ohne FEMtech hätte sie wahrscheinlich nicht dissertiert."

Damit kann in Zusammenhang mit der Programmlinie FEMtech Dissertationen durchaus von Additionalitätswirkungen gesprochen werden und zwar in zweierlei Hinsicht: zum einen werden Frauen dazu motiviert, eine Dissertation zu verfassen - etwas was sie ohne die FEMtech Förderung mitunter nicht getan hätten - und damit ihre Karrierechancen im Bereich der Naturwissenschaft/Technik zu verbessern. Zum anderen trägt die Programmlinie - zumindest in einigen Fällen - auch dazu bei, dass in Unternehmen bzw. Forschungseinrichtungen bestimmte Projekt überhaupt erst ermöglicht bzw. auf höherem wissenschaftlichen Niveau durchgeführt werden, als es ohne die Förderung der Fall gewesen wäre.

„Ohne FEMtech Dissertationen wäre das Projekt brach gelegen. Wir hätten das Thema ohne das Programm nicht weiterverfolgt. Durch die Förderung haben wir eine kleine Forschungsgruppe zu dem Thema aufbauen können. Jetzt ist es eine langfristige Richtung, die wir eingeschlagen haben und über FEMtech Talente hinaus weiterführen werden." 
„Das Projekt wäre wahrscheinlich auch ohne Förderung durchgeführt worden, aber in einem deutlich reduzierten Ausmaß nur intern (von unserem Industriepartner). FEMtech hat dazu beigetragen, dass das Projekt wissenschaftlich in Form einer Dissertation gemacht wird."

\section{Unterstützung von Nachwuchswissenschaftlerinnen}

Die Dissertantinnen werden von den geförderten Unternehmen und Forschungseinrichtungen sehr umfassend betreut. Dies umfasst die Unterstützung im technischen Bereich (z.B. Durchführung von Experimenten, Interpretation von Daten, Anleitung zur Berichtserstellung, troubleshooting etc.) sowie die Teilnahme an Ausund Weiterbildungsmaßnahmen, Summer Schools, wissenschaftlichen Konferenzen, internen Schulungen und Seminaren etc. In allen befragten Unternehmen bzw. Forschungseinrichtungen nehmen die Dissertantinnen an derartigen Maßnahmen und Aktivitäten teil bzw. ist eine entsprechende Teilnahme geplant.

Darüber hinaus bestehen oftmals auch langjährige Kontakte der Projektnehmerlnnen zu den BetreuerInnen der Dissertation auf der jeweiligen Universität, die sich in einigen Fällen auch an der Definition des Projektes bzw. der Antragstellung beteiligt haben. Dies trägt - insbesondere bei Unternehmen - dazu bei, dass kaum „Zielkonflikte“ zwischen den Anforderungen der Unternehmen bzw. Forschungseinrichtungen an das Projekt sowie etwaigen (wissenschaftlichen) Vorgaben seitens der Universität auftreten.

Insbesondere innerhalb der geförderten Forschungseinrichtungen scheinen die Dissertantinnen dabei auch sehr gut in die jeweiligen Forschungsteams bzw. die Organisation als solches integriert zu sein. Für Unternehmen dürfte dies hingegen mitunter eine große Herausforderung darstellen. Einer der ProjektnehmerInnen, ein Unternehmen, war beispielsweise zum Zeitpunkt des Interviews gar nicht sicher, ob die Integration überhaupt gelingen würde. Darüber hinaus wird die Aufnahme einer Dissertantin von diesem Unternehmen, das bisher nur Diplomarbeiten betreut hat, als sehr betreuungsintensiv und zeitaufwendig wahrgenommen. Dementsprechend wird die Förderquote in diesem Fall auch als zu niedrig empfunden.

\section{Einstiegsmöglichkeiten ins Berufsleben}

Durch FEMtech Dissertationen soll den Dissertantinnen eine Einstiegsmöglichkeit in das Berufsleben der angewandten Forschung - konkret im Bereich Produktion und Material - geboten werden. Auch dazu leistet die Programmlinie einen wesentlichen Beitrag, zumindest was die Projekte der ersten Ausschreibungsrunde betrifft. Bei drei der ProjektnehmerInnen ist die entsprechende Dissertantin bereits in Vollzeit bzw. zu 5/6 angestellt (z.T. sogar unbefristet), bei den anderen beiden ist eine Weiterbeschäftigung der Dissertantin nach Projektende geplant bzw. grundsätzlich vorstellbar. Damit dürfte diesen Frauen der Einstieg ins Berufsleben nicht zuletzt auch dank FEMtech Dissertationen - bereits erfolgreich geglückt sein. 


\section{Allgemeine Beurteilung der Programmlinie}

Die Programmlinie FEMtech Dissertationen wird von den ProjektnehmerInnen durchwegs sehr positiv beurteilt. Als Stärke der Programmlinie wird dabei die Möglichkeit gesehen, den wissenschaftlichen Nachwuchs zu fördern, wobei die Meinungen darüber, was die Einschränkung auf den weiblichen Nachwuchs betrifft durchaus auseinandergehen. Während einige der Projektnehmerlnnen der ersten Ausschreibung die ausschließliche Förderung von weiblichen Studierenden als zu rigoros bzw. diskriminierend empfinden, geht es für andere bei FEMtech Dissertationen eben in erster Linie um die Förderung von Frauen in Naturwissenschaft und Technik, mit dem Ziel, den Frauenanteil in diesen Bereichen zu erhöhen. Mit der Öffnung der Programmlinie für Männer führt sich FEMtech Dissertationen aus ihrer Sicht ad absurdum, da es ja genau darum gehe, die Möglichkeiten und Chancen von Frauen im naturwissenschaftlich-technischen Bereich zu verbessern. Ein Projektnehmer formuliert das sehr deutlich: „Die Öffnung für Herren halte ich für Unfug, die findet man eh leicht".

Alle Unternehmen und Forschungseinrichtungen, die im Rahmen der ersten Ausschreibungsrunde gefördert wurden, könnten sich jedenfalls vorstellen, erneut bei FEMtech Dissertationen einzureichen (ein Projektnehmer sieht jedoch eine Erhöhung der Förderquote als Voraussetzung für eine erneute Teilnahme). 


\subsection{Talente finden}

\subsubsection{Karriere-Grants}

Die Programmlinie Karriere Grants unterstützt ForscherInnen, die eine Beschäftigung am Forschungsstandort Österreich anstreben. Förderbar sind hierbei Reisekosten für Vorstellungsgespräche, Umzugskosten bei Stellenantritt und die berufliche Integration des/der Partners/Partnerin.

Tabelle 7 Eckpunkte von Karriere Grants

\begin{tabular}{|l|l|}
\hline Karriere-Grants & Kurzbeschreibung \\
\hline Ziele & $\begin{array}{l}\text { Unterstützung ausländischer ForscherInnen bei ihrem Karriere- } \\
\text { sprung nach bzw. bei der Fortsetzung der Karriere in Österreich. }\end{array}$ \\
\hline Zielgruppen & Einzel-ForscherInnen bzw. deren Partnerlnnen aus dem Ausland. \\
\hline Inhalt & $\begin{array}{l}\text { Förderung von Forscherlnnen im Ausland die nach Österreich kom- } \\
\text { men, um Vorstellungsgespräche (im Bereiche FEl) durchzuführen } \\
\text { bzw. neue Beschäftigungsverhältnisse anstreben, sowie die berufli- } \\
\text { che Integration des/der Partnerln. }\end{array}$ \\
\hline Einreichverfahren & $\begin{array}{l}\text { Laufende Einreichung (Interviewgrants spätestens ein Monat nach } \\
\text { dem Bewerbungsgespräch, Relocation Grants drei spätestens drei } \\
\text { Monate nach der Übersiedelung, Dual Career Grants spätestens } \\
\text { sechs Monate nach der Übersiedelung), wobei die Formalkriterien } \\
\text { vorab geprüft werden. }\end{array}$ \\
\hline Faufzeit & $\begin{array}{l}\text { Förderungen werden anlassbezogen bezogen geprüft und ausbe- } \\
\text { zahlt. }\end{array}$ \\
\hline $\begin{array}{l}\text { 80\% der Reise- und Nächtigungskosten für Vorstellungsgespräche } \\
\text { in Österreich, Relocation Grant (max. € 2.000), Dual Career Grant } \\
\text { (max. € 2.000). }\end{array}$ \\
\hline
\end{tabular}

Seit Beginn des Karriere-Grants Förderangebotes im Oktober 2011 haben insgesamt 323 ForscherInnen und deren PartnerInnen Anträge zur Unterstützung gestellt. Davon fallen 33 in das Förderjahr $2011^{24}, 146$ sind 2012 gestellt worden und 144 sind dem Jahr 2013 zuzurechnen. Zur tatsächlichen Auszahlung von Förderbeträgen kam es in 29 (2011), 130 (2012) bzw. 90 (2013) Fällen, wobei vor allem die Durchführung von Bewerbungsgesprächen und die Übersiedelung des Wohnsitzes gefördert wurden (jeweils rd. 50 \%) (siehe Grafik 10). Dual Career Grants wurden insgesamt in elf Fällen von den mitübersiedelnden Partnerlnnen beantragt, jedoch in nur drei tatsächlich ausbezahlt. Der angestrebte Wert von insgesamt 75 vergebenen Grants pro Jahr konnte somit im Untersuchungszeitraum (2012 bis 2013) erreicht werden.

24 Da die Programmlinie der Karriere Grants erst im 4.Quartal des Jahre 2011 in das Förderangebot der FFG aufgenommen wurde, ist die Vergleichbarkeit diese Jahres nur bedingt gegeben. In weiterer Folge stehen daher die Untersuchungsjahre 2012 und 2013 im Vordergrund der Betrachtungen. 


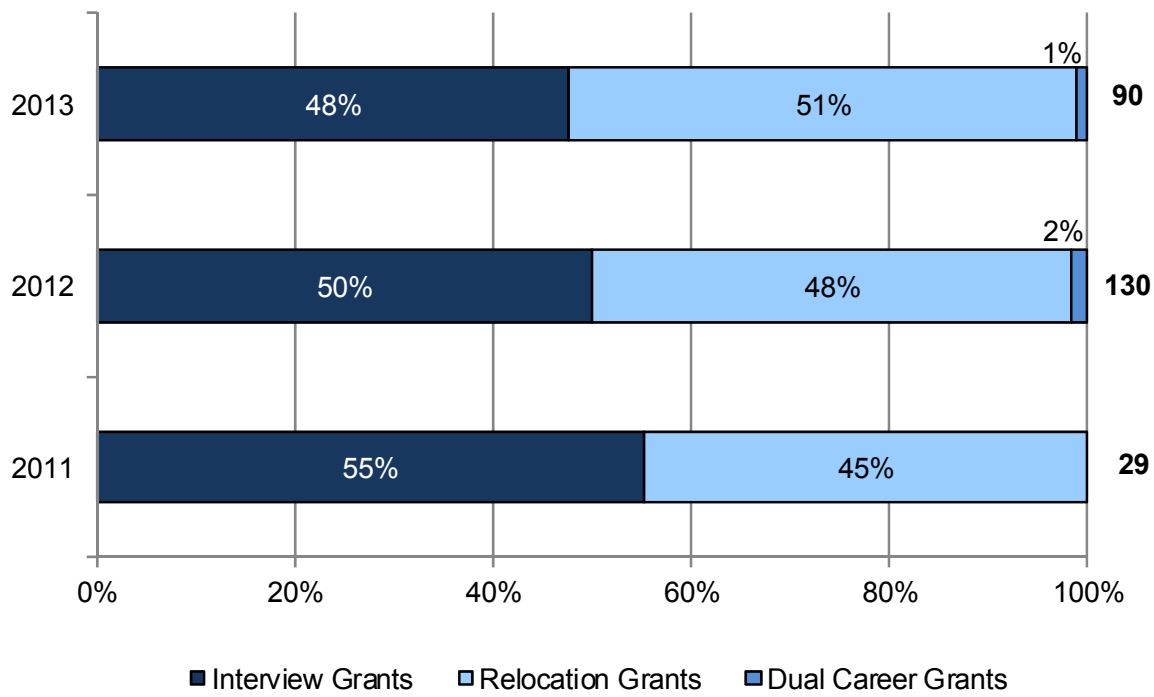

Quelle: FFG-Monitoringdaten

Wenig überraschend wurden mehr als drei Viertel der beantragten Grants von ForscherInnen eingereicht, die sich bereits in Europa aufgehalten haben. Die übrigen waren mehrheitlich in Nordamerika (11\%) und Asien (8\%) tätig, bevor sie einen Wechsel ihres Forschungsstandortes nach Österreich angestrebt haben. Diese Verteilung ist auch bei den tatsächlich ausgezahlten Karriere Grants erkennbar. Betrachtet man die unterschiedlichen Nationalitäten der ForscherInnen zeigen sich hingegen leichte Abweichungen von der Verteilung der Forschungsstandorte. Zwar ist der Anteil der europäischen Nationalitäten mit $80 \%$ weiterhin dominierend, ForscherInnen asiatischer Herkunft bewerben sich jedoch deutlich häufiger (14\%) als ihre Nordamerikanischen KollegInnen (4\%). Des Weiteren waren rd. zwei Drittel, sowohl der einreichenden als auch der geförderten Forscherlnnen, männlich.

$38 \%$ der Forscherlnnen, die mittels Interview Grant gefördert wurden, haben die betreffende Forschungsstelle in Österreich erhalten bzw. angenommen. Die Stelle nicht bekommen haben rd. $43 \%$ der entsprechenden Forscherlnnen bzw. haben $6 \%$ die Stelle nicht angenommen. In den verbliebenen $13 \%$ war eine endgültige Entscheidung noch ausstehend.

Der Großteil der ausbezahlten Karriere Grants entfiel auf Forschungsstellen auf universitärer bzw. FH-Ebene (48 \%), gefolgt von Unternehmen (31 \%) und außer- 
universitären Forschungseinrichtungen (20\%). Die verbliebenen Karriere Grants waren keiner Organisationsart zuordenbar (Dual Career Grant).

Demensprechend hoch ist auch der Anteil der angenommen Forschungsstellen an Universitäten und FHs (54 \%). Rund ein Drittel der ForscherInnen, die ein Karriere Grant erhalten haben, hat eine unternehmensinterne Forschungsstelle angenommen und $15 \%$ wurden in außeruniversitären Forschungseinrichtungen angestellt.

Von den im Rahmen von Karriere Grants geförderten Forscherlnnen wurden $48 \%$ bei der Bewerbung bzw. Übersiedelung nach Wien unterstützt. $15 \%$ der genehmigten Fördermittel wurden für BewerberInnen von Vorarlberger Forschungsstellen aufgebracht, weitere $12 \%$ entfielen auf in der Steiermark angestrebte Stellen. Der Rest verteilt sich zum überwiegenden Teil auf Niederösterreich (10\%), Oberösterreich (7\%) Salzburg und Tirol (jeweils rd. $3 \%$ ).

\subsubsection{Jobbörse}

Die Österreichische Jobbörse für Forschung, Entwicklung und Innovation ist eine frei zugängliche und kostenlos nutzbare Plattform, welche die Zusammenführung von Jobanbietern und -suchenden im F\&E- sowie Innovationsbereich ermöglicht. ${ }^{26}$ Auch Praktikumsplätze können über die Jobbörse vergeben werden.

Die Nutzungszahlen der Jobbörse haben sich Zeitraum von 2011 bis 2013 wie folgt entwickelt: im 4. Quartal des Jahres 2011 wurden insgesamt 931 Jobangebote in der Jobbörse veröffentlicht. Im ersten Halbjahr des Jahres 2012 wuchs dieser Wert auf mehr als das Doppelte an und erreichte im 2. Quartal 2012 seinen Höhepunkt. Die tagesdurchschnittliche Zahl der veröffentlichten Jobangebote verläuft erwartungsgemäß ähnlich und erreichte einen Spitzenwert von 533 im 3. Quartal 2012. Im weiteren Verlauf nahm die Zahl der angebotenen Stellen wieder deutlich ab. Schließlich wurden Ende des Jahres 20131.031 Jobangebote in der Jobbörse angeboten, wobei der Tagesdurchschnitt der angebotenen Stellen bei einem Wert von 333 lag. Insgesamt betrachtet ist die Gesamtzahl der angebotenen Stellen im Untersuchungszeitraum um rd. 13.000 Einträge angestiegen (siehe Grafik 11).

26 Für die nachfolgenden Betrachtungen ist zu beachten, dass es im Jahr 2012 zu einer Neuprogrammierung der Jobbörse kam und die Vergleichbarkeit der verbundenen Monitoring-Zeitreihen daher nur eingeschränkt möglich ist. 
Grafik 11 Gesamtanzahl und Tagesdurchschnitt der veröffentlichten Jobangebote im Zeitablauf

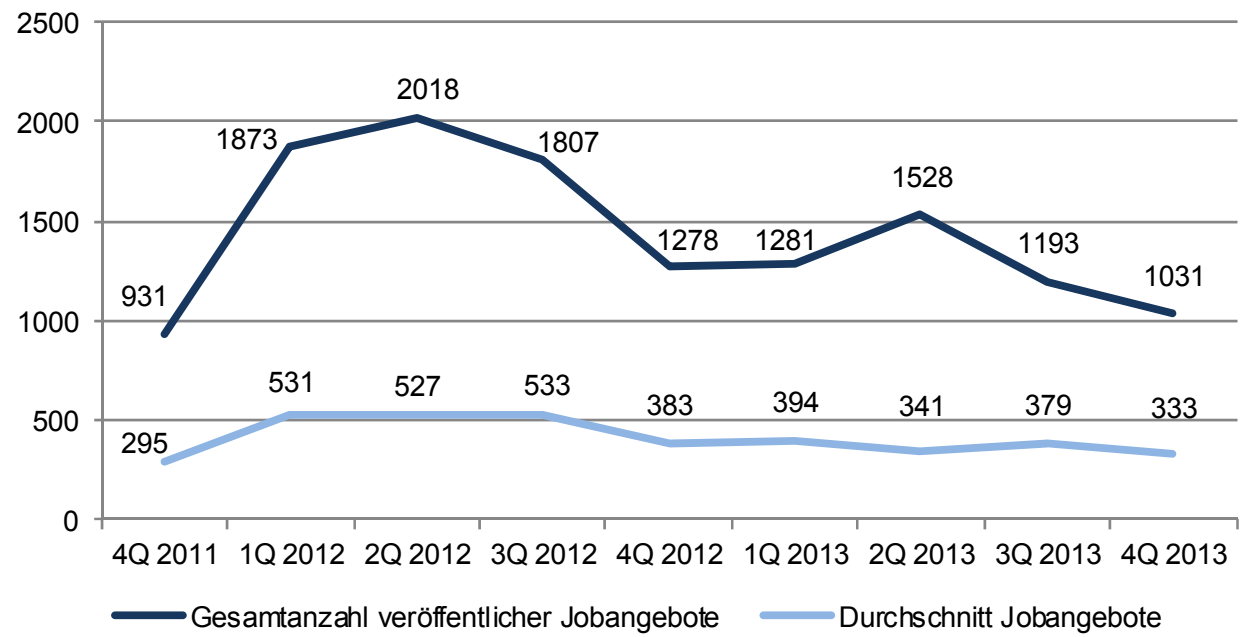

Quelle: FFG Quartalsstatistiken Jobbörse

Die sinkende Anzahl der angebotenen Stellen drückt sich auch in den sinkenden Seitenaufrufen bzw. den Unique Visitors (HomepagebesucherInnen) aus. Von Anfang 2013 bis Jahresende haben sich die Seitenaufrufe nahezu halbiert, während die Zahl der BesucherInnen um rd. $18 \%$ zurückgegangen ist. Die angestrebte Steigerung der Nutzung der Jobbörse ${ }^{27}$ kann daher zumindest für diesen Zeitraum nicht festgestellt werden.

Differenziert nach Fachgebieten zeigt sich bei den Jobangeboten ein deutlicher Überhang im Bereich der technischen Wissenschaften. Zwischen $75 \%$ und $86 \%$ der angebotenen Stellenausschreibungen sind in technischen und naturwissenschaftlichen Forschungsfeldern konzentriert. Weitere Fachgebiete sind Medizin, Geistes- und Kulturwissenschaften sowie Sozial-, Wirtschafts- und Rechtswissenschaften, wobei diese lediglich einstellige Prozentanteile der Jobangebote ausmachen.

Die regionale Verteilung der offerierten Stellenangebote kann lediglich von 2012 bis 2013 dargestellt werden und zeigt nur marginale Schwankungen. Die meisten Ausschreibungen sind Unternehmen und F\&E-Einrichtungen aus Wien zuzurechnen, rd. $40 \%$ der Stellen entfielen auf dieses Bundesland. Weitere $20 \%$ stammten aus der Steiermark bzw. rd. $10 \%$ aus Tirol. Auf die verbleibenden Bundesländer entfielen im Jahr 2013 Stellenangebote in niedrigen Prozentbereichen. Darüber hinaus war der Großteil der Stellenangebote im Bereich Forschung und Entwick-

${ }^{27}$ Programmdokument und Sonderrichtlinien Talente - Der Förderschwerpunkt des BMVIT 
lung organisiert (96\%), während es sich bei nur $4 \%$ der Angebote um Innovationsjobs handelte.

Die Nutzung der Jobbörse durch unterschiedliche Organisationstypen ist vom 4.Quartal 2011 bis zum 3. Quartal 2012 durch die verstärkte Inanspruchnahme durch Unternehmen gekennzeichnet. Diese konnten ihren Anteil auf rd. ein Drittel der Einträge verdoppeln. Gleichzeitig ist der Anteil der universitären Stellenangebote von $44 \%$ auf $37 \%$ bzw. der außeruniversitäre Forschungseinrichtungen von $24 \%$ auf $18 \%$ zurückgegangen ${ }^{28}$.

\subsection{Begleitmaßnahmen}

\subsubsection{FEMtech Netzwerktreffen}

Als Begleitmaßnahme der FEMtech Programmlinien werden regelmäßig Netzwerktreffen organisiert, die zum Ziel haben, Neuigkeiten aus dem Förderschwerpunkt zu verbreiten, neue Studien aus dem Themenbereich Gender in Wissenschaft und Forschung vorzustellen und aktuelle Themen in diesem Zusammenhang zu diskutieren. Zielgruppe sind Nachwuchsforscherinnen, Forscherinnen, Unternehmen und Forschungseinrichtungen sowie Stakeholder. Jedes Netzwerktreffen bietet auch Gelegenheit zum informellen Austausch und zur Förderberatung durch die FFG. Im Zeitraum 2011 bis 2013 fanden folgende Netzwerktreffen statt:

- „Forscherinnen fördern heißt: Alle Talente nützen“ 20. Juni 2011, Wien

- "Macht - schillernd und handfest. Anleitungen zum strategischen Spiel“ 21. November 2011, Wien

- „Chancenland Vorarlberg. Das gesamte Potenzial nützen. Erfolg durch Vielfalt"

18. Juni 2012, Dornbirn

- "Chancengleichheit und Exzellenz, eine Frage der Forschungskultur" 19. November 2012, Wien

- „Unternehmenskulturen verändern - mehr Frauen in Führungspositionen“ 24. Juni 2013, Linz

- „Innovative Mischung - Lösung zur Überwindung von Diskriminierung“ 28. Oktober 2013, Wien

Das Format der Netzwerktreffen wurde vom Programm FEMtech übernommen.

28 Aufgrund der Neuprogrammierung der Jobbörse kam es im 4.Quartal 2012 zu einer veränderten Reportingfunktion, weshalb der Vergleich mit den Vorquartalen nicht mehr möglich ist. 


\subsubsection{Tickets für Talente@Alpbach}

Im Jahr 2013 wurden erstmals sogenannte Alpbach-Tickets vergeben. Studierenden aus Naturwissenschaft und Technik wird dabei die Teilnahme an den Alpbacher Technologiegesprächen ermöglicht. Die Tickets beinhalten die kostenlose Teilnahme an den Technologiegesprächen sowie zusätzliche für die Studierenden organisierte Events (z. B. Workshops mit ForscherInnen, Möglichkeit des Treffens mit PolitikerInnen und Führungskräften österreichischer Top-Technologie-Unternehmen). Es werden auch die Unterbringungskosten sowie ein Teil der Reise- und Verpflegungskosten übernommen.

Für die insgesamt 50 Tickets bewarben sich im Jahr 201385 StudentInnen. 24 Studentinnen und 26 Studenten erhielten ein sog. Alpbach-Ticket. Die meisten geförderten StudentInnen stellten die TU Wien (17) und die Universität Wien (12). Mehr als die Hälfte der geförderten TeilnehmerInnen an den Technologiegesprächen war zwischen 22 und 25 Jahre alt. 


\section{Gesamtbetrachtung und Schlussfolgerungen}

Der Förderschwerpunkt Talente bündelt die Förderungen des BMVIT im Bereich Humanpotenzial und fokussiert auf den gesamten Karriereverlauf von ForscherInnen im weitesten Sinn, d. h. es werden (potentielle) ForscherInnen im Kindesalter ebenso gefördert wie etablierte ForscherInnen. Die drei Interventionsfelder mit ihren acht Programmlinien decken alle Karrierestationen ab und die verschiedenen Programmlinien ergänzen einander zu einer „Förderkette“. Dabei wird jede „Karrierestation" (mit Ausnahme der Vorschulkinder) von mindestens zwei Programmlinien adressiert.

Auch sprechen die Programmlinien alle potentiellen Fördernehmerlnnen an. Von insgesamt knapp 1.200 Organisationen, die Förderungen aus dem TalentePortfolio in Anspruch genommen haben, sind etwas mehr als die Hälfte Unternehmen, $17 \%$ Universitäten und Fachhochschulen und $8 \%$ außeruniversitäre Forschungseinrichtungen. ${ }^{29}$ Bei Betrachtung der Verteilung nach Projektteilnahmen ${ }^{30}$ sinkt der Anteil der Unternehmen auf knapp unter $50 \%$, während der Anteil von außeruniversitären Forschungseinrichtungen auf $22 \%$ und jener von Universitäten und Fachhochschulen auf $21 \%$ steigt (siehe Grafik 20 im Anhang). Daraus wird ersichtlich, dass sich insbesondere die außeruniversitären Forschungseinrichtungen an mehreren Programmlinien bedienen bzw. im Rahmen einer Programmlinie mehrere Förderungen in Anspruch nehmen. Dies zeigt, dass auch die FördernehmerInnen den Förderschwerpunkt als Angebot einer Förderkette begreifen. So haben $12 \%$ der FördernehmerInnen zwei oder mehr Programmlinien in Anspruch genommen und $33 \%$ haben mehrere Förderungen innerhalb einer Programmlinie erhalten. Meist werden Praktika für SchülerInnen oder FEMtech Praktika für Studentinnen mit einer anderen Programmlinie kombiniert, auch bei mehrfacher Inanspruchnahme einer Programmlinie sind es überwiegend Praktika für SchülerInnen.

Zudem scheint der Förderschwerpunkt mit seinen teils sehr niederschwelligen Angeboten (v. a. Praktika für SchülerInnen, Praktika für Studentinnen) ein „Einsteigerprogramm" zu sein. $17 \%$ der FördernehmerInnen haben im Rahmen von Talente das erste Mal eine Förderung bei der FFG in Anspruch genommen. ${ }^{31}$ Zwei Drittel dieser Erstförderungen finden sich bei der Programmlinie Praktika für SchülerInnen, weitere $16 \%$ bei Talente regional, $15 \%$ bei Praktika für Studentinnen und $3 \%$ bei FEMtech Forschungsprojekte (siehe Grafik 21 im Anhang).

\footnotetext{
${ }^{29}$ Die restlichen rd. $20 \%$ "Sonstige" sind in erster Linie EinzelforscherInnen, die im Rahmen von Karriere-Grants gefördert werden. Quelle: FFG-Monitoringdaten

${ }^{30}$ Dies sind rd. 3.200, d. h. hier wird die Organisation pro Projektteilnahmen gezählt.

${ }^{31}$ Wenn man die Karriere-Grants hinzuzählt, die sich an Einzelforscherlnnen richten, und auf eine einmalige Förderung ausgelegt sind, sind es sogar 33 \%. Quelle: FFG-Monitoringdaten
} 
Da der Förderschwerpunkt unter der Annahme etabliert wurde, dass die Humanpotenziale für die Forschung grundsätzlich verfügbar sind, aber unzureichend ausgeschöpft werden, kommt vor dem Hintergrund der Unterrepräsentanz von Frauen dem Genderthema besondere Bedeutung zu. Sichtbar wird dies zum einen durch die Integration des Programms FEMtech, zum anderen über die Vorgabe der Berücksichtigung von Genderkriterien auch in anderen Programmlinien (Talente regional, Praktika für Schülerlnnen, FEMtech Dissertationen). Die Berücksichtigung des Genderthemas auf einer inhaltlichen Ebene wird durch die Integration von FEMtech Forschungsprojekte betont, das als einzige Programmlinie nicht die Förderung von Humanressourcen zum Ziel hat, sondern genderrelevante Forschungsprojekte fördert. Dadurch wird insgesamt die Wichtigkeit und Relevanz der Thematik unterstrichen und strukturelle Änderungen können initiiert werden. Wenn Forschungsinhalte "gegendert" werden, liegt es nahe, dies auch in der Organisation zu tun.

Insgesamt ist zudem ersichtlich, dass die meisten Instrumente eher auf Personen abzielen (und dadurch auch größere Breitenwirkung haben), einige wenige aber auch auf strukturelle Änderungen (in erster Linie FEMtech Karriere, aber auch Talente regional). Um die Förderung von Humanressourcen nachhaltig als Ziel auch bei den relevanten AkteurInnen (Unternehmen, Forschungseinrichtungen) zu verankern, könnte bei den FördernehmerInnen von Einsteigerformaten verstärkt „Werbung“ für diese auf Strukturänderungen abzielenden Programmlinien gemacht werden.

Im Folgenden wird über die einzelnen Programmlinien ein Resümee gezogen sowie mögliche Interventionsfelder diskutiert:

\section{Praktika für Schülerinnen und Schüler}

Die Praktika für Schülerinnen und Schüler stellen ein breitenwirksames Instrument zur Förderung von potenziellen Nachwuchsforscherlnnen dar. Die zunehmende Inanspruchnahme seitens der SchülerInnen, die positive Gesamtbeurteilung bezüglich erhaltener Einblicke in den naturwissenschaftlich-technischen Forschungsbereich und das verstärkte Interesse an den relevanten Forschungsfeldern sowie die Zufriedenheit der FördernehmerInnen zeigen die Relevanz und Akzeptanz des Instruments auf. Da jedoch bei der Inanspruchnahme der Praktika Jugendliche aus technischen Schultypen (HTL) dominieren, bleibt einerseits das Potenzial von Nachwuchsforscherlnnen aus der AHS ungenützt bzw. wird andererseits die noch stärkere Einbindung weiblicher Nachwuchsforscherinnen erschwert (obwohl es gelungen ist, bereits ein Drittel der Praktika an Mädchen zu vergeben). Da die Schullaufbahnentscheidung bezüglich der Oberstufe aber mitten in die Pubertät fällt (Mitte der 8. Schulstufe), in der genderstereotype Entscheidungen durch die verstärkte Identitätsfindung wahrscheinlich sind, sollte verstärktes Augenmerk auf die SchülerInnen gerichtet werden, deren Weg noch nicht in Richtung eines naturwissenschaftlich-technischen Studiums oder eines Berufs in diesem Bereich vor- 
gezeichnet ist. Da zudem in den HTL Pflichtpraktika vorgesehen sind, in der AHS aber nicht, sollte diesem Schultyp verstärktes Augenmerk zukommen. Umfassende Informationsverbreitung in den Schulen sowie die Forcierung der Nutzung der Praktikabörse (sowohl durch die SchülerInnen als auch durch die Fördernehmerlnnen) könnte zu einer größeren Ausgewogenheit bei den PraktikantInnen sowie in regionaler und fachlicher Sicht führen.

\section{Talente regional}

Die Programmlinie Talente regional wird von den beteiligten Akteurlnnen als sehr geeignetes und notwendiges Instrument gesehen, um Kinder und Jugendliche für Naturwissenschaft und Technik zu begeistern. Diese konnten ihre Kenntnisse in diesem Bereich erweitern und haben Einblicke in zukunftsträchtige Berufsfelder erhalten. Aber auch die ProjektpartnerInnen dürften von der Teilnahme profitiert haben. Neben nachhaltiger Vernetzung profitieren die wissenschaftlichen Partnerlnnen davon, ihre Tätigkeiten einem breiteren Publikum zu vermitteln und regional bekannter zu werden. Die Unternehmenspartnerlnnen sehen in der Teilnahme an diesen Projekten v. a. eine langfristige Recruitingmöglichkeit. Die PädagogInnen erweiterten ihre Kenntnisse in unterschiedlichen Bereichen und können nun auch auf für das jeweilige Projekt erarbeitete Materialien und Hilfsmittel zurückgreifen, die sie u. a. in Projektarbeit integrieren können. Daher verwundert auch nicht, dass Talente regional nicht nur weiterempfohlen wird, sondern meist auch ein neues Projekt mit einem ähnlichen Projektkonsortium eingereicht wurde.

Aus den Herausforderungen derartiger kooperativer Projekte ergeben sich dennoch mögliche Optimierungsvorschläge. Die Koordination erweist sich ob der vielen involvierten Partnerlnnen als sehr aufwendig, und alle Befragten berichten davon, dass der Aufwand in keinem Verhältnis zur Förderung stehe. Daher wird eine Erhöhung des Budgets angeregt bzw. die Möglichkeit dem Projektmanagement einen höheren Budgetanteil zuordnen zu können. Die Kooperationszuschüsse werden grundsätzlich begrüßt, zur Flexibilisierung des Instruments wird jedoch angeregt, statt strikt zehn Mal $€ 1.000$,- vergeben zu müssen, auch die Möglichkeit zu haben, z. B. nur fünf Kooperationszuschüsse zu $€ 2.000$,- zu vergeben.

Inhaltlich liegt die Herausforderung bei Talente regional in der zielgruppenadäquaten Aufbereitung der Themen, Materialien, Experimente etc. für die verschiedenen Altersstufen. Zurzeit ist die Einbindung von drei Bildungsstufen verpflichtend vorgesehen. Eine Reduzierung auf zwei Bildungsstufen würde die Projekte vereinfachen (auch bezüglich der Akquise der Bildungspartnerlnnen) und könnte zu noch passgenaueren Angeboten führen. 
Organisatorisch am problematischsten scheint die Integration der Talente regional Projekte in das Schuljahr bzw. in den Schulalltag. Der Zeitpunkt der Ausschreibung bzw. der Förderentscheidung sollte so gewählt werden, dass der Projektbeginn schon im Frühjahr erfolgen kann, sodass die Projekte von Beginn an in der Planung des neuen Schuljahres berücksichtigt werden können.

Da in einem Talente regional Projekt sehr viel Entwicklungsarbeit steckt, könnte in Erwägung gezogen werden, erfolgreiche Projekte mit ähnlichem Projektinhalt in einer neuerlichen Ausschreibung zuzulassen, wenn diese andere Bildungspartner einbinden oder in einer anderen Region tätig werden.

\section{FEMtech Forschungsprojekte}

Mit den FEMtech Forschungsprojekten ist es gelungen, die Genderrelevanz von Forschungsprojekten technischer Themenbereiche in das Zentrum der Förderung zu rücken und damit eine Lücke des vorherrschenden Förderungsbedarfs zu schließen. Angebot und Umsetzung orientieren sich dabei - den geführten Interviews und den geförderten Forschungsprojekten nach zu urteilen - an Themen mit einem hohen gesellschaftlichen Nutzen und stoßen dabei auf zunehmendes Interesse seitens potenzieller Fördernehmerlnnen. Darüber hinaus stärkt das Vorhandensein der Förderlinie die Wahrnehmung der Genderrelevanz und ermöglicht die stärkere Sensibilisierung des Themas in technischen Forschungsfeldern.

Obwohl es sich hier um klassische Forschungsförderung handelt, hat die Programmlinie insofern ihren Platz im Talente-Portfolio, als von einer ausstrahlenden Wirkung der Inhalte auf das Forschungsfeld und die Organisationen, die dieses Feld bearbeiten, ausgegangen werden kann. Da die beteiligten Organisationen schon über große Genderkompetenz verfügen (müssen), sollten diese als Role Models genützt werden, um zu zeigen, dass eine Verankerung von Gender Mainstreaming in der Organisation auch zu einem Kompetenzaufbau im Forschungsfeld führen kann. Solange genderrelevante Forschungsinhalte nicht in ausreichendem Maße in anderen Programmlinien Berücksichtigung finden, scheint eine Integration in Talente unabdingbar.

\section{FEMtech Karriere}

Im Rahmen von FEMtech Karriere konnten nicht so viele Projekte gefördert werden, wie als Zielsetzung vorgegeben war und somit die bereitgestellten Mittel auch nicht ausgeschöpft werden. Dieser geringen Nachfrage nach dem Förderinstrument stehen die FördernehmerInnen gegenüber, die sich von der Möglichkeit begeistert zeigen, ein Personalentwicklungsprojekt in dieser Breite durchführen zu können. Die hohe Additionalitätswirkung, Fördernehmerlnnen, die schon zum zweiten Mal ein FEMtech Karriere Projekt durchführen, sowie die in den meisten Fällen sehr konkreten Ergebnisse der Projekte, die zu einer breiten Verankerung von Gender Mainstreaming in den Organisationen führen, sprechen für die Qualität des Instruments. 
Anzumerken ist, dass fast alle FördernehmerInnen schon Genderexpertise aufweisen, sei es auf institutioneller Ebene oder auf personeller Ebene, in der Person der Projektleitung. FEMtech Karriere scheint daher ein Instrument für Organisationen, in denen Gender Mainstreaming bzw. Personalentwicklung schon ein Thema ist.

Um einen breiteren Adressatenkreis ansprechen zu können, könnte die Programmlinie adaptiert bzw. gesplittet werden. FEMtech Karriere "light" könnte erste Maßnahmen zur Implementierung von Chancengleichheit fördern, ohne dass ein Gesamtkonzept zur Verankerung von Gender Mainstreaming in der gesamten Organisation vorgelegt werden muss. Hier könnte z. B. nur ein Modul gefördert werden sowie die Förderung (und der Eigenmittelanteil) entsprechend geringer ausfallen. Dies würde v. a. die Barrieren für NeueinsteigerInnen bzw. KMU, senken. „Fortgeschrittene" Fördernehmerlnnen könnten wie bisher ein volles FEMtech Karriere Projekt beantragen, bei einem Folgeantrag müsste dann aber gesichert sein, dass ein neuer Fokus und neue Elemente ins Projekt integriert werden.

In jedem Fall wären die Maßnahmen zur Verbreitung der Fördermöglichkeit zu erhöhen, wobei die Wirkungen der Implementierung von Maßnahmen zur Erhöhung der Chancengleichheit zu zeigen sind (Good Practice Bespiele), v. a. unter „unbedarften“ potentiellen FördernehmerInnen.

\section{FEMtech Studentinnenpraktika}

Das enorme Interesse der FördernehmerInnen und Studentinnen an dem Förderangebot führte zu der vorzeitigen Mittelausschöpfung in den bisherigen Ausschreibungsrunden. Die sichtbar hohe Nachfrage birgt - unter Berücksichtigung der angeführten Weiterbeschäftigungsanteile und Additionalitäten - zusätzliches Potenzial für ausgeweitete kurz- und langfristige Anstellungen von Studentinnen im Forschungsbereich und somit den möglichen Einstieg in eine Karriere in der Forschung in sich. Darüber hinaus zeigt die Förderung hohen inhaltlichen Nutzen, sowohl auf Seiten der Studentinnen, die durch die Praktika ein deutlich gesteigertes Interesse an naturwissenschaftlich-technischer Forschung aufweisen, als auch auf Seiten der Unternehmen, die von den positiven Wirkungen auf die Forschungsaktivitäten überzeugt sind und die Möglichkeit der Rekrutierung von Mitarbeiterinnen nützen wollen, aber meist erst durch die Förderung Praktikumsstellen anbieten können.

Da die Studentinnen durch die einschlägige Studienwahl schon einen Schritt in Richtung Naturwissenschaft/Technik gemacht haben, aber die endgültige Berufswahl noch aussteht, scheinen Faktoren, die die Motivation im Berufsfeld zu bleiben, wie sie ein Praktikum darstellt, entscheidend, um dem Phänomen der leaky pipeline (Frauen gehen im Karriereverlauf der Forschung verloren) zu begegnen. Daher sollte eine Aufstockung der Mittel für die FEMtech Praktika für Studentinnen in Erwägung gezogen werden. 


\section{FEMtech Dissertationen/Dissertationen in den Themen des BMVIT}

Die Programmlinie FEMtech Dissertationen zielt auf die Höherqualifizierung von (weiblichen) Nachwuchswissenschaftlerlnnen ab und weist in Hinblick auf ihre verfolgten Zielsetzungen ein hohes Potenzial auf. Sie ist durch hohe Additionalitätswirkungen sowohl auf Seiten der DissertantInnen als auch in Bezug auf die geförderten Unternehmen und Forschungseinrichtungen gekennzeichnet und wird durch die Betroffenen durchwegs sehr positiv beurteilt. Die Motivation für eine Projektteilnahme ist dabei oftmals in erster Linie nicht durch den Wunsch geprägt, gezielt Nachwuchswissenschaftlerlnnen zu fördern, sondern vielmehr ein bestimmtes Thema (wissenschaftlich) zu bearbeiten. Dadurch gelingt es im Rahmen von FEMtech Dissertationen auch bis dato in Bezug auf das Thema Gender eher „unbedarfte" Unternehmen und Forschungseinrichtungen anzusprechen und sie für die gezielte Förderung von Frauen in Naturwissenschaft und Technik zu sensibilisieren. Hier sind durch die Programmlinien, insbesondere im Rahmen der ersten Ausschreibung, die sich ausschließlich an den weiblichen Nachwuchs richtete, wichtige Schritte gesetzt worden.

Dementsprechend kontrovers wird auch die Öffnung der Programmlinie für Männer im Zuge der zweiten Ausschreibung beurteilt, die bereits aufzeigt, wie wichtig die Einführung einer 50\%-Quote für Frauen war, um zu verhindern, dass bei der Suche nach geeigneten DissertantInnen nicht ausschließlich auf den im naturwissenschaftlich-technischen Bereich leichter zu findenden männlichen Nachwuchs zurückgegriffen wird. Zu prüfen wäre in Zusammenhang mit FEMtech Dissertationen nur, in wie weit eine Anhebung der Förderquote für Unternehmen, die erstmals eine Dissertantin / einen Dissertanten aufnehmen bzw. für KMU sinnvoll bzw. notwendig sein könnte, da diese oftmals über wenig Erfahrung in der Durchführung wissenschaftlicher Projekte verfügen und somit einen deutlich höheren Betreuungs- und Organisationsaufwand haben, als Forschungseinrichtungen.

\section{Karriere-Grants}

Die Karriere-Grants wurden im Rahmen der Zwischenevaluierung nur mittels Monitoringdaten analysiert. Es können daher keine Aussagen über Akzeptanz, Zufriedenheit der FördernehmerInnen und Additionalitätswirkungen etc. getroffen werden. Diese wären in einer etwaigen Endevaluierung des Förderschwerpunkts zu prüfen.

\section{Jobbörse}

Auch die Jobbörse wurde nicht näher analysiert. In einer Endevaluierung des Förderschwerpunkts wäre zu untersuchen, wie die Nutzerlnnen diese beurteilen und wie eine höhere Nutzung der Jobbörse erreicht werden könnte. 
Insgesamt scheint die Bündelung der Aktivitäten des BMVIT im Bereich Humanressourcen gelungen und notwendig, um die Wichtigkeit des Themas für die Forschung an sich zu unterstreichen, denn es gilt, dass es ohne exzellente Forscherlnnen auch keine exzellente Forschung geben kann. Zur Sichtbarmachung des Förderschwerpunkts Talente und um eine noch größere Breitenwirksamkeit zu erzeugen, erscheint es wichtig, Talente als Marke stärker zu etablieren und den Förderschwerpunkt in seiner Gesamtheit sowie die einzelnen Programmlinien entsprechend zu promoten. 
6. Anhang

6.1. Fallstudien 


\section{Fallstudie Talente regional: fly DOO}

\section{Projektteam}

Das Konsortium wurde vom RIC (Regionales Innovations Centrum), einem Kooperationsnetzwerk in Gunskirchen in Oberösterreich, angeführt. Partnerlnnen waren die BRP-Powertrain $\mathrm{GmbH}$ \& Co KG (75\% Eigentümer von RIC), die FH OÖ Campus Wels und Conrad Electrics $\mathrm{GmbH}$. Als Bildungspartnerlnnen fungierten der Kindergarten Gunskirchen, die Volksschulen Gunskirchen und Bad Wimsbach, die Hauptschulen Gunskirchen, Eberstalzell, Pichl und Steinerkirchen, die polytechnischen Schulen (PTS) Schwanenstadt und Grieskirchen, die AHS Wels Dr. Schauerstraße, die HTL Wels sowie die BRP-Powertrain Lehrwerkstätte.

\section{Ziele und Motivation}

Das Talente regional Projekt "fly DOO“ sollte Kinder/Jugendliche für Naturwissenschaft/Technik im Allgemeinen sowie die Luft- und Raumfahrt im Besonderen begeistern. Dabei sollten Berufsmöglichkeiten in diesen Bereichen aufgezeigt werden und über das Ansprechen von MultiplikatorInnen (LehrerInnen, Eltern) auch langfristiges Recruiting für diesen Bereich gefördert werden.

Das Innovationszentrum, das in laufendem Kontakt mit der FFG steht, versteht sich als Innovations- und Bildungsmotor und hat große Erfahrung in der Durchführung von Jugendprojekten. Da auch enge Verbindungen zu SchulpartnerInnen der Region bestanden, schien eine Programmlinie wie Talente regional ideal, um das Interesse für Kinder und Jugendliche zu wecken. Zudem ist in der BRP-Powertrain $\mathrm{GmbH} \&$ Co KG das Thema Diversity großgeschrieben. So soll der Anteil weiblicher Lehrlinge von $25 \%$ auf $50 \%$ erhöht werden. Als Weltmarktführer für Flugzeugmotoren lag ein Thema rund um das Fliegen auf der Hand.

Im Fokus des Projekts stand auch der Wissenstransfer zwischen Wirtschaft und Schule über die „Recruitierung der LehrerInnen, die keine Unternehmen von innen kennen". Es sollte das Bild von technischen Berufen, die oftmals mit schwerer körperlicher Arbeit und Schmutz etc. verbunden werden, geändert und die Unternehmenskultur kennengelernt werden, damit ein realitätsnäheres Bild an die Schülerlnnen weitergetragen werden kann („wir arbeiten mit High-Tech Maschinen, Sauberkeit und Sicherheit sind selbstverständlich“).

\section{Projektdurchführung}

Im Rahmen einer Kick-off Veranstaltung an der $\mathrm{FH}$ wurden alle Beteiligten über das Projekt, wichtige Abläufe und die ProjektpartnerInnen informiert. Die Eltern erfuhren, in welche Bereiche ihre Kinder eingebunden sein werden und konnten auch die FH kennenlernen. 
In Workshops mit PädagogInnen wurden erste Kenntnisse in Mechatronik anhand eines Flugmodells erläutert. Es galt, den PädagogInnen Einblick in den Alltag eines Lehrlings zu geben, weibliche Role Models zu präsentieren und die Wichtigkeit der LehrerInnen als Multiplikatorlnnen hervorzuheben, damit diese ihre Erfahrungen in die Klassen tragen konnten, um so zu einer Horizonterweiterung in Hinblick auf die Berufswahl von Jugendlichen beizutragen.

Danach starteten die Vorbereitungsphasen für den Kindergarten sowie die Volkschulen und Hauptschulen. Kindergartenkinder wurden zu einer Exkursion zum Flughafen Linz eingeladen, bei der auch kleine praktische Übungen durchgeführt wurden. Die Schülerlnnen der Volksschulen wurden eingeladen, im Zuge der KinderUni Steyr am Training „Faszination Fliegen“ teilzunehmen. Im Werkunterricht wurde das Thema Luft- und Raumfahrt bearbeitet und die Kinder hatten die Möglichkeit, an der HTL Wels einer Windkanalübung beizuwohnen. Zudem wurden die Eltern in die BRP-Powertrain Lehrwerkstätte eingeladen. In den Hauptschulen wurde das Thema fächerübergreifend im Werkunterricht, in Physik und Biologie bearbeitet und die HauptschülerInnen konnten die HTL besuchen.

Weiterführende Workshops mit Lehrerlnnen der HS und AHS an der FH über mechanische und strömungsmechanische Grundlagen des Baus von Fluggeräten, in denen das Thema altersadäquat für die jeweiligen SchülerInnen aufbereitet und Hands-on für den Unterricht bereitgestellt wurde, schlossen die Vorbereitungsphase ab.

Danach wurden die Schülerlnnen der Volks- und Hauptschule in der praktischen Phase in die Lehrwerkstätte eingeladen, praktische Übungen durchzuführen und Modellflugzeuge zu bauen. Die Kindergartenkinder bauten eigene Drachen und Raketen. Schülerlnnen der höheren Schulen wurden angeleitet, eigenständig ein Segelflugzeug zu bauen. Dieses wurde in der Folge mit einem Elektromotor ausgestattet. Da nicht in jeder Schule die Voraussetzungen für die Konstruktion von Elektromotoren gegeben waren, wurden schulübergreifende Teams zusammengeführt, die jeweils Elektrosegler bauten. Diese konnten in einer ersten Flugshow getestet werden und wurden in der abschließenden AIR SHOW einem breiten Publikum präsentiert.

Alle beteiligten Klassen und Gruppen erhielten Preise (Modellbaukit, Flüge über Wels etc.), die zwei besten und fleißigsten Schülerlnnen erhielten einen zweiwöchigen Aufenthalt im NASA Space Camp im Juli 2013.

\section{Kooperation}

Die Zusammenarbeit mit den ProjektpartnerInnen lief laut Projektleitung problemlos, allerdings kannte man sich auch schon in den meisten Fällen. Zur Wahrung der Kontinuität scheint die Mischung aus alten und neuen PartnerInnen bei neuen Projekten am erfolgversprechendsten. 
Die Kooperation mit den Schulen verlief grundsätzlich sehr positiv, allerdings musste der Fokus auf die persönliche Betreuung gelegt werden. Die Einbindung in das laufende Schuljahr ist herausfordernd, daher sollte beim Timing der Ausschreibung berücksichtigt werden, dass die Lehrerlnnen ein Talente regional Projekt ins neue Schuljahr integrieren können.

Bei den Kooperationszuschüssen war zunächst kein Ansturm zu beobachten, aber schließlich konnten alle zehn Zuschüsse vergeben werden, fünf Anträge wurden sogar abgelehnt.

\section{Wirkungen}

Laut Projektleitung zeigten sich die involvierten Kinder/Jugendlichen sehr begeistert von den Aktivitäten. Zwei der Jugendlichen haben sich in der Folge für einen technischen Lehrberuf entschieden, was sie ohne dieses Projekt nicht getan hätten, eine Teilnehmerin aus einer höheren Schule will Luft- und Raumfahrt studieren.

Die PädagogInnen konnten ExpertInnenwissen nützen und haben erkannt, dass Aktivitäten aus einem solchen Projekt zu einer Bereicherung des Unterrichts führen können. Außerdem wurde viel Anschauungsmaterial erarbeitet, das auch in der Folge genützt werden kann. Durch das Projekt kam es zudem zu einem sehr intensiven Austausch zwischen den Bildungsinstitutionen in der Region. Im Zuge des Projekts wurde auch der Fortbildungskurs "Mechatronics for Teachers" entwickelt und in den Bildungskatalog des Landes aufgenommen.

Eine große Herausforderung stellte es dar, die Zielgruppen (PädagogInnen, Eltern, Kinder) „abzuholen“, d.h. diesen soweit entgegenzukommen, um überhaupt Begeisterung für das Thema und das Projekt wecken zu können. Das hieß bei den LehrerInnen das Thema soweit aufzubereiten, dass es in den Unterricht integriert werden kann. So stellten sich die Vorbereitungs-Workshops mit den PädagogInnen als unabdingbar heraus, um so ein Projekt erfolgreich zu gestalten.

\section{Lessons learnt}

$\mathrm{Da}$ die ProjektpartnerInnen beobachten, dass das Interesse von Kindern/ Jugendlichen an Technik eher im Abnehmen begriffen scheint, sind diese von der Notwendigkeit von Initiativen wie Talente regional überzeugt und erachten diese als wichtige CSR-Maßnahme. Das Konzept der Programmlinie wird als absolut geeignet gesehen, Interesse bei der Zielgruppe zu wecken.

In diesem Zusammenhang wird auch betont, dass die Arbeit mit den Multiplikatorlnnen als sehr wichtig für den Erfolg eines solchen Projekts gesehen wird. Daher ist das Timing entscheidend, um ein solches Projekt auch gut ins Schuljahr integrieren zu können.

Der Konsortialführer hat mit einem Teil der Partnerlnnen von „fly DOO“ in der dritten Ausschreibungsrunde ein neues Projekt eingereicht. 


\section{Fallstudie Talente regional: FreiRaumKlima Talente Mureck}

\section{Projektteam}

Im Projekt FreiRaumKlima Talente Mureck hatte der Verein Green City Lab e.V. die Projektleitung inne und wurde von den wissenschaftlichen PartnerInnen Universität für Bodenkultur Wien (BOKU) / Institut für Landschaftsplanung, Verkehrplus $\mathrm{GmbH}$ und Research and Data Competence OG unterstützt. Die (vor)schulischen BildungspartnerInnen waren die HLW Mureck, die BAKIP Mureck, die Polytechnische Schule Mureck, die Hauptschule Mureck, die Volksschule Mureck sowie der Übungskindergarten Mureck in der Steiermark.

\section{Ziele und Motivation}

Im Mittelpunkt des Projekts standen die Themen Freiraum, Mobilität und Energieeffizienz. Das abstrakte Thema Klimawandel sollte mit der Erfahrungswelt von Kindern und Jugendlichen verknüpft werden. Ziel des Projekts war es,

- das Interesse an planerischen Fragestellungen in Naturwissenschaft und Technik zu wecken,

- zukunftsträchtige Arbeitsfelder für Frauen und Männer (Stichwort Green Jobs) zu vermitteln,

- den Klimawandel anhand von Fragestellungen, die das Living Environment betreffen, erfahrbar, messbar und begreifbar zu machen,

- die Bewusstseinsbildung und Sensibilität für den Klimawandel zu erhöhen,

- das Thema Gender in der Konzeption, Durchführung und Evaluierung des gesamten Projekts zu berücksichtigen und

- die Vernetzung zwischen (vor-)schulischen Bildungseinrichtungen und (außer-)universitären Forschungseinrichtungen zu fördern.

Die Projektleiterin erfuhr über Mundpropaganda von Talente regional und formte ein Konsortium mit ProjektpartnerInnen, die sie aus dem Mobilitätsforschungsbereich kannte und die auch schon mit Jugendlichen gearbeitet haben. Die Bildungseinrichtungen wurden über Kontakte zur Gemeinde angesprochen. Neben der Wichtigkeit, Kindern und Jugendlichen die Themen Klima und Klimawandel näher zu bringen, sahen die Projektleiterin und die Verantwortliche an der BOKU mit dem Projekt auch die Möglichkeit, ein didaktisches Konzept von der Universität an einer anderen Klientel und verschiedenen Bildungsstufen zu erproben und umzusetzen. Zudem hatte die BOKU so Zugang zu einer Nutzergruppe, die normalerweise in Planungsprozessen aufgrund der schweren Erreichbarkeit kaum berücksichtigt wird. 


\section{Projektdurchführung}

Das Projekt folgte der didaktischen Leitlinie des forschenden Lernens. Den Kindern und Jugendlichen wurden keine fertigen Konzepte für ein Forschungsprojekt vorgelegt, sondern die konkreten Fragestellungen wurden erst gemeinsam mit der Zielgruppe erarbeitet. Leitfrage war: Wie können wir in Mureck und Umgebung 2050 mit hoher Lebensqualität leben, arbeiten und unterwegs sein? Wie können wir unser Verhalten und die Gestaltung von Freiräumen an sich ändernde Rahmenbedingungen anpassen?

In einem Startworkshop wurde die Detailplanung mit den betroffenen Pädagoglnnen besprochen und diskutiert, wie mit den Kindern/Jugendlichen die konkreten Fragestellungen erarbeitet werden können. An insgesamt drei Aktionstagen ging das Forschungsteam in die Bildungseinrichtungen, um mit der Zielgruppe gemeinsam zu forschen und zu experimentieren. Insgesamt waren rd. 200 Kinder/Jugendliche in das Talente regional Projekt involviert. Es wurde ein Stationen- und TutorInnensystem errichtet, bei dem jüngere Schülerlnnen durch ältere begleitet wurden. Zwischen den Aktionstagen bearbeiteten die Kinder/Jugendlichen die Forschungsfragen weiter. Es wurden z. B. Umfragen und Messreihen durchgeführt, Handstromgeräte gebaut oder Fahrräder zu E-Bikes umgebaut. Zudem wurde ein Plan zur Umgestaltung des Schulgartens erarbeitet. Schülerlnnen der HLW haben auf der Basis vorangegangener Erhebungen und Befragungen den Busbahnhof umgestaltet und organisierten das Sponsoring für die Materialien. Den Abschluss des Projekts bildete ein Forschungsfest, bei dem alle beteiligten SchülerInnen ihre Arbeiten vorstellen konnten. Die Anwesenheit von Eltern, Gemeindevertreterlnnen und MedienvertreterInnen sorgte für eine Verbreitung der Ergebnisse und die Schülerlnnen konnten sich als „Teil einer Forschungscommunity“ fühlen.

Das Projekt war auch durch das Bemühen gekennzeichnet, die Genderkomponente durchgehend zu berücksichtigen. Dies geschah zum einen durch die Berücksichtigung geschlechtsspezifischer Unterschiede bei Untersuchungsdesigns und Messreihen, zum anderen bei der Bildung reiner Mädchen- und Bubengruppen beim Forschen und Experimentieren. Dies erwies sich v. a. in der Hauptschule als notwendig, um die Mädchen zu einer aktiveren Teilnahme zu bewegen.

\section{Kooperation}

Die Projektorganisation erfolgte sehr arbeitsteilig. Jede/r Partnerln war für die Ausgestaltung in der Schulklasse verantwortlich und stimmte die Planung mit den anderen KollegInnen ab. Die Kooperation war durch gegenseitige Wertschätzung und die Bereitschaft, die anderen PartnerInnen zu unterstützen, gekennzeichnet. 
Die Zusammenarbeit mit den Lehrerlnnen gestaltete sich dagegen schwieriger. V. a. die Integration des Projekts in das - schon zum Großteil verplante - Schuljahr stellte eine große Herausforderung dar. Die PädagogInnen konnten zum Teil auch nur schwer abschätzen, welchen inhaltlichen Input und wie viele zeitliche Ressourcen das Projekt ihrerseits benötigen würde.

Als Erfolgsfaktoren für eine gelungene Zusammenarbeit wurden von den ProjektpartnerInnen die klare Rollenaufteilung und eine transparente Kommunikation (auch über Protokolle) genannt. Wichtig erscheint auch ein gemeinsames Verständnis der Ziele und Inhalte, „fachlich waren wir uns einig“. Zentral sind auch geeignete Personen, „die mit Kindern können“, Forscherln zu sein qualifiziere noch nicht zur Teilnahme an so einem Projekt. Die erfolgreiche Kooperation wird auch in der dritten Ausschreibungsrunde, in der in ähnlicher Partnerkonstellation ein weiteres Talente regional Projekt eingereicht wurde, fortgesetzt.

Im Rahmen des Projektes wurden auch zehn Kooperationszuschüsse vergeben. Hier berichtete die Projektleiterin von teilweise sehr hohem Informations- und Aufklärungsbedarf („das war ein bisschen mühsam“). Oft mussten die Anträge der PädagogInnen für die FFG überarbeitet werden. Irritationen entstanden auch, weil die Auszahlung auf das Privatkonto der PädagogInnen erfolgt.

\section{Wirkungen}

Bei den Jugendlichen und Kindern konnte eine große Begeisterung bei den Aktionstagen beobachtet werden. Die Zielgruppe gewann einen Einblick in die Forschung und in Berufsbilder in der Naturwissenschaft und Technik. Auch wurde beobachtet, dass über die Forschungstätigkeiten umweltbewusstes Handeln angeregt werden konnte. V.a. das alltagsorientierte Forschen scheint seine Spuren bei den Kindern/Jugendlichen hinterlassen zu haben. Am wirksamsten haben sich jedenfalls die Aktivitäten im Projekt herausgestellt, bei denen das selber Ausprobieren im Vordergrund stand. Auch die Diskussion mit den WissenschaftlerInnen und die Herausforderung, selber relevante Forschungsfragen zu formulieren, hat die Zielgruppe sehr motiviert.

In der Region wurden die Schulen als aktive Gestalterinnen wahrgenommen und das Projekt erhielt breite Resonanz auch in den regionalen Medien.

Auch die BOKU berichtet von dem Projekt profitiert zu haben. Die involvierte Forscherin erzählte, dass sie ihre „Einstellung zu den StudentInnen kalibrieren konnte". Sie hat wieder Einblicke in den Schulalltag bekommen, der ihr beim Verständnis für die Erstsemestrigen helfe und sie nun wisse, über welche Themen sie den StudentInnen Theorien und Methoden näher bringen kann. 


\section{Lessons learnt}

Grundsätzlich wird die Programmlinie Talente regional als sehr geeignet erachtet, Kindern/Jugendlichen die Welt der Forschung und Naturwissenschaft/Technik näher zu bringen. Als Stärken werden die Offenheit bezüglich der Themen und Herangehensweise genannt und die Langfristigkeit der Projekte, die eine Begleitung der Zielgruppe über ein ganzes Schuljahr erlaubt und punktuellen Maßnahmen gegenüber überlegen ist („kein Ein-Tages Event"). Auch die Vernetzung mit Leitbetrieben in der Region erscheint als wesentlicher Erfolgsfaktor.

Gleichzeitig wird Talente regional als sehr herausfordernd beschrieben. Es enthält viele Ansprüche, insbesondere in Hinblick auf die Einbindung so vieler verschiedener Bildungsstufen, die alle altersadäquat betreut werden sollen. Zudem ist ein kooperatives Projekt mit großem organisatorischen Aufwand verbunden, hinsichtlich der Koordination der PartnerInnen im Allgemeinen und mit den Schulen (Integration in den Schulalltag und in die Planung des Schuljahres, administrative Erfordernisse bezüglich außerschulischer Aktivitäten, Lehrplan versus Projektinhalte etc.) im Besonderen. Vor diesem Hintergrund wird das maximale Projektbudget als bescheiden und v.a. die Beschränkung auf max. 20\% der Gesamtkosten für das Projektmanagement als zu gering erachtet.

Trotz dieser Kritikpunkte überzeugte das Konzept von Talente regional die Projektleitung und einige Partner derart, dass diese in der dritten Ausschreibung wiederum ein Projekt eingereicht haben. 


\section{Fallstudie Talente regional: LlchtQuadrat}

\section{Projektteam}

Das Weizer-Energie-Innovations-Zentrum (W.E.I.Z.) führte das Konsortium mit der Energieagentur Ing. Baierl, dem wissenschaftlichen Partner Joanneum Research MATERIALS und den Pichlerwerken Elektrotechnik an. Involvierte Schulen waren die HTL, die HLW, die Sporthauptschule und die Neue Mittelschule Weiz sowie die Volksschule Weizberg. Zudem waren die Sozialeinrichtungen "Lebenshilfe Weiz" und "Christina lebt" in das Projekt involviert.

\section{Ziele und Motivation}

Oberstes Ziel des Talente regional Projekts "LlchtQuadrat" war es, ganzheitliche Beleuchtungskonzepte, die gesellschaftlich-soziale, rechtliche, technologische, wissenschaftliche, betriebswirtschaftliche und ökologische Aspekte berücksichtigen, auf optimierter und nachhaltiger Basis von jungen Talenten und in intensiver, vernetzter Zusammenarbeit zwischen fünf Weizer Schulen mit insgesamt 125 SchülerInnen, der Wissenschaft, der Wirtschaft und dem Sozialwesen zu erarbeiten, umzusetzen und so nachhaltig Kosteneinsparungen für die beiden sozialen Einrichtungen zu ermöglichen.

SchülerInnen der HTL und HLW sollten einen praxisnahen Bezug zu Technik und Wirtschaft erhalten und sehen, wie Wirtschaftsprojekte im Berufsleben abgewickelt werden und dass Forschung ein wichtiger Bestandteil für technische Innovationen ist. Zudem sollten die älteren Schülerlnnen den Kindern und Jugendlichen der HS, NMS und Volksschule durch Peer-Tutoring-Aktivitäten ihr erlerntes Wissen in Lichtund Beleuchtungstechnik weitervermitteln.

Im Aufgabenbereich von W.E.I.Z. liegt die Positionierung des Standorts und da das Innovationszentrum, die Energieagentur und Joanneum Research schon ein Vorgängerprojekt in einem ähnlichen Themenbereich gemeinsam durchgeführt haben, lag es nahe, bei Talente regional einzureichen. Für den wissenschaftlichen Partner bedeutete das Projekt eine Möglichkeit, durch den direkten Kontakt zur Bevölkerung, das Institut und seine Forschungstätigkeit einem breiteren Publikum näherzubringen und in der Region bekannter zu werden. Damit sollten auch potentielle NachwuchsforscherInnen angesprochen werden. Die Pichlerwerke erwarteten sich durch die Teilnahme direkten Zugang zu Jugendlichen, den sie als bedeutender Lehrlingsausbildner in der Steiermark intensivieren wollten. 


\section{Projektdurchführung}

Im Arbeitspaket „Bildungs- \& Forschungsinitiative Beleuchtung“ standen nach Vorgesprächen mit PädagogInnen in allen teilnehmenden Schulen je zwei Workshops auf dem Programm, in denen fachtheoretischer und experimenteller Unterricht kombiniert wurde. Die ForscherInnen von Joanneum Research vermittelten zunächst theoretisches Wissen in interaktiven Unterrichtsformaten, bei denen die Fragestellungen und die Aufgabenstellungen der jeweiligen Altersgruppen angepasst wurden. Der Schwerpunkt lag jedoch beim aktiven Forschen, Experimentieren und beim praktischen Arbeiten mit Licht bzw. zum Thema Beleuchtung. Alle SchülerInnen führten angeleitet in Kleingruppen die gleichen Experimente aber je nach Alter in unterschiedlichen Detailierungsgraden durch. Zudem konnten fast alle Schülerlnnen die Forschungslabors von Joanneum Research kennenlernen.

Die erste Praxisinitiative „Beleuchtung Ist“ beinhaltete eine Bestandsaufnahme in den Sozialeinrichtungen. Die Beleuchtungssituation und die Energieverbrauchsdaten wurden mit den WirtschaftspartnerInnen durch Rundgänge und mit Plänen erhoben und die gesammelten Daten von den Schülerlnnen analysiert und ausgewertet.

In der zweiten Praxisinitiative „Beleuchtung Soll“ sollten zwei ganzheitliche Beleuchtungskonzepte für die Sozialeinrichtungen auf Basis der Ist-Analyse erstellt werden. Die Konzepte wurden im Rahmen einer Maturaarbeit von Schülerinnen der HLW Weiz erstellt, wobei gesellschaftlich/soziale, rechtliche, technologisch/wissenschaftliche, betriebswirtschaftliche und ökologische Aspekte behandelt wurden. Auch der Punkt „Finanzierung“ wurde berücksichtigt, und nach Vorbereitung durch die WirtschaftspartnerInnen führten Schülerlnnen der HTL und der HLW Preisverhandlungen mit drei Anbieterlnnen und konnten $25 \%$ Rabatt, $5 \%$ Skonti und Materialsponsoring beim Bestbieter erreichen.

Neben der Konzepterstellung standen in diesem Arbeitspaket auch Peer-Tutoring Aktivitäten im Mittelpunkt. Jeweils vier SchülerInnen der HTL unterrichteten gemeinsam eine Klasse der HS und der NMS und vier HLW-Schülerlnnen fungierten als „LehrerInnen“ in zwei Volksschulklassen. Damit wurde auch die schulübergreifende Zusammenarbeit gefördert.

Die dritte Praxisinitiative „Beleuchtung Neu“ hatte zum Ziel, die Beleuchtungskonzepte umzusetzen. Es wurden Spenden- und Sponsorengelder lukriert und es erfolgte eine von den Pichlerwerken betreute Umrüstung der Beleuchtung in den Sozialeinrichtungen. Dabei konnten viele Schülerlnnen der HS und NMS aktiv Hand anlegen und die Tätigkeiten von Elektro- und Lichttechnikerlnnen kennenlernen. Kompliziertere Optimierungsarbeiten wurden mit HTL-Schülern durchgeführt.

Das gesamte Projekt wurde durch eine professionelle Medienarbeit begleitet und beinhaltete neben der Errichtung einer Facebook-Seite u. a. regelmäßige Bericht- 
erstattung in der Stadtzeitung Weiz, eine Auftakt-Pressekonferenz, aber auch die Organisation der Spendenaktion.

\section{Kooperation}

Ein One-Stop-Shop LlchtQuadrat sollte die regelmäßige Kommunikation zwischen den ProjektpartnerInnen mittels Projektmeetings und regelmäßigem Austausch sichern. Die Projektleitung wird von den Beteiligten als sehr professionell bezeichnet.

Als schwieriger hat sich die Koordination mit den Schulen herausgestellt, da hier eine langfristige Planung gefragt und der Informationsbedarf vorab sehr hoch ist. Dennoch haben sich viele LehrerInnen gefunden, die sich in hohem Maße einbrachten und immer wieder Zeit vom Unterricht „abzwackten“, um dem Projekt ausreichend Raum zu geben. Joanneum Research berichtet von sehr positivem Feedback der PädagogInnen, die auch vielfach die Materialien (z. B. Experimentierkoffer) weiter im Unterricht verwenden wollen. Dies dürfte nicht zuletzt auch an der Persönlichkeit der ForscherInnen liegen, die laut Aussage anderer Projektpartnerlnnen Begeisterung für dieses Projekt zeigten und diese auch der Jugend weitergaben.

\section{Wirkungen}

Die Jugendlichen waren von den Aktivitäten angetan. Vor allem der von Joanneum Research entwickelte Experimentierkoffer stieß auf große Begeisterung („,da lebt es dann") und motivierte zur weiteren Beschäftigung mit dem Thema. V. a. auch Mädchen waren sehr motiviert. Die Schülerlnnen der HTL und HLW zeigten sehr viel Verantwortungsbewusstsein sowohl beim Unterrichten der jüngeren Schülerlnnen als auch bei der praktischen Durchführung des Projekts für die Sozialeinrichtungen. Es konnten praktische Erfahrungen aus dem zukünftigen Berufsleben gemacht werden (Beleuchtungskonzepte erstellen, Preisverhandlungen führen, Umrüstungsarbeiten ausführen). Das Peer-Tutoring stellte sich dabei als voller Erfolg dar. VolksschülerInnen fanden den Unterricht "cool" und ein HTL-Schüler beschloss in der Folge, den Lehrerlnnenberuf anzustreben.

Zudem scheint durch das Talente regional Projekt ein verstärktes Umweltbewusstsein bei den Kindern/Jugendlichen hervorgerufen worden zu sein. Diese agieren nun als MultiplikatorInnen und tragen ihre Erkenntnisse in Bezug auf das Energiesparen ins Elternhaus, zu Freunden etc. Damit werden die Ideen, für die das Projekt steht, weitertransportiert.

Die ProjektpartnerInnen schätzten die Möglichkeit für ein solches Netzwerkprojekt und haben nun ein herzeigbares Referenzprojekt. Der wissenschaftliche Partner konnte seine Tätigkeit hinaustragen und erfahrbar machen. Zudem wurden ein Programm und Experimente entwickelt, die nunmehr auch mit anderen Schulen durchgeführt werden können. Das Projekt brachte den Partnerlnnen einen noch 
besseren Zugang zum Nachwuchs, z. B. zu zukünftigen Lehrlingen. W.E.I.Z. konnte seine Kompetenz als Innovationszentrum bestätigen.

Bei den Sozialeinrichtungen konnten Energie- bzw. $\mathrm{CO}_{2}$-Einsparungen von $46 \%$ bzw. 28 \% durch die Umrüstung auf LED-Technologie erzielt werden. Damit wurde dem Anspruch des Projekts, Umweltbewusstsein mit sozialem Engagement zu paaren, gerecht.

Das Projekt bedeutet auch Werbung für die Region und da die HTL ein Einzugsgebiet weit in andere Teile der Steiermark hat, werden die Projektideen auch in andere Regionen getragen.

\section{Lessons learnt}

Zu den Stärken des Projekts bzw. der Programmlinie Talente regional zählt es, dass hier die Möglichkeit geschaffen wird, Forschung und Wissenschaft erfahrbar zu machen - eine Voraussetzung, um Kinder/Jugendliche dafür zu begeistern. Talente regional hat laut den Interviewten ein "tolles Konzept" und scheint auch besonders geeignet, um Mädchen für Naturwissenschaft und Technik zu begeistern.

Als Voraussetzung für den Erfolg werden griffige Ideen und die inhaltliche Homogenität des Projekts genannt. Zudem sind die Harmonie zwischen den PartnerInnen, ein koordiniertes Vorgehen und ein gutes Projektmanagement unabdingbar. Unter diesen Voraussetzungen ist eine neuerliche Teilnahme an einem Talente regional Projekt für die Befragten in jeden Fall denkbar. 


\section{Fallstudie Talente regional: Technologykids}

\section{Projektteam}

Das Konsortium des Projekts Technologykids wurde vom gleichnamigen Verein als Projektleiter angeführt. Als wissenschaftliche Partnerlnnen fungierten die Fachhochschule Wiener Neustadt, Standort Tulln, das Biomin Research Center und die Romer Labs. Involvierte Bildungseinrichtungen waren der NÖ Landeskindergarten 7, das Volkshilfe Kinderhaus Tulln, die Volksschule 1 aus Tulln, die Volksschule Konigstetten sowie die NMS Marc Aurel in Tulln.

\section{Ziele und Motivation}

Ziel des Projekts war die „Steigerung des Interesses an Naturwissenschaft/Technik durch Hands-on und Role Models“. Zu diesem Zweck sollte auf die Gendergerechtigkeit in der Umsetzung geachtet, die Vernetzung der Schulen und Kindergärten mit Institutionen und Firmen am Technopol Tulln vorangetrieben, ein wissenschaftlicher Beirat gebildet und ein Forschungsfest am Campus Tulln etabliert werden.

Die Projektleiterin hatte den Wunsch, mit Kindern im Bereich Naturwissenschaft/Technik zu arbeiten und auf der Suche nach einer Finanzierung stieß sie auf die Programmlinie Talente regional und gründete den Verein Technologykids. Da sie am Technopol Tulln beschäftigt ist, war das Konsortium über persönliche Kontakte rasch formiert, wobei sich die Suche nach den BildungspartnerInnen etwas schwieriger gestaltete. Die UnternehmenspartnerInnen sahen die Mitarbeit im Projekt als PR-Maßnahme, die FH sah darin eine Möglichkeit, in der Region bei potentiellen StudentInnen und MultiplikatorInnen bekannter zu werden („Es ist wichtig, dass wir bekannter werden als Fachhochschule, es wissen gar nicht so viele, dass es diese Möglichkeit gibt, in Tulln zu studieren in dem Bereich.")

\section{Projektdurchführung}

Nach einer Vorbereitungsphase, in der über das Forschen mit Kindern recherchiert wurde, wurde das konkrete Arbeitsprogramm ausgearbeitet. Dies beinhaltete die altersadäquate Ausgestaltung von Versuchen, Arbeitsblättern und Arbeitsanweisungen und die Bereitstellung der Materialien. Die Programme wurden vorab mit den PädagogInnen diskutiert.

Das Praxisprogramm begann zunächst in den Bildungseinrichtungen: Forscherlnnen der FH und der Unternehmenspartner besuchten die Kindergärten und Schulen, um die anschließenden Exkursionen vorzubereiten. Bei den Besuchen in den Forschungslabors führten dann meist bis zu drei Kinder ein Experiment durch und wurden dabei von einer/m Forscherln unterstützt. Inhaltlich wurde v. a. mit Lebensmitteln experimentiert, z. B. Inhaltsstoffe analysiert oder mittels Molekularküche „Fruchtsaftkaviar" hergestellt. Dabei sollten alle Kinder aktiv eingebunden werden und keines in der ZuschauerInnenrolle verharren. 
Beim abschließenden Forschungsfest am Campus Tulln konnten nicht nur die Schulklassen, die in das Projekt involviert waren, an verschiedenen Stationen forschen und experimentieren, sondern es konnten auch Schulklassen, die in das Talente regional Projekt der zweiten Ausschreibung „MicroNature - Technologykids erforschen den Mikrokosmos" eingebunden sind, sowie KooperationszuschussempfängerInnen teilnehmen (insgesamt rd. 250 Kinder und Jugendliche). Beispiele für Stationen waren „Mobiles Bodenlabor", „Wasser schmeckt“, „Gummibärli-DNA“ oder „Vom Feld in den Stall“.

Als zentraler Erfolgsfaktor, um die Zielgruppe anzusprechen, wird das Experimentieren durch die Kinder gesehen. „Hingreifen, selbst Hand anlegen, selber mikroskopieren" scheint essentiell, um das Interesse der Kinder zu wecken. Auch alles, was mit Natur zu tun hat, spricht die Kinder an. Zu vermeiden ist dagegen zu viel Theorievermittlung.

\section{Kooperation}

Die Kooperation stand von Anfang an auf einem stabilen Fundament, da diese über das persönliche Netzwerk der Projektleiterin zustande kam. Die Informationen sind zentral bei der Projektleitung zusammengelaufen. Diese fungierte auch als zentrale Anlaufstelle, was Klarheit in der Kommunikation brachte. Nach dem Kickoff Meeting wurde auf große Meetings verzichtet, aber es gab eine Vielzahl an bilateralen Gesprächen zwischen Projektleitung und Mitgliedern der Umsetzungsgruppe. Die erfolgreiche Partnerschaft wird auch in einem Talente regional Projekt der zweiten Ausschreibungsrunde in ähnlicher Partnerkonstellation fortgesetzt.

Die Zusammenarbeit mit den Bildungspartnerlnnen gestaltete sich unterschiedlich. Während es mit Kindergärten und Volksschulen keinerlei Probleme gab, weil diese eine große zeitliche Flexibilität mitbringen, ist die Koordination mit einer NMS wesentlich komplizierter. Diese haben ein sehr enges zeitliches Korsett und bei einer mehr als einstündigen Exkursion müssen auch mehrere Fachlehrerlnnen koordiniert werden.

Voraussetzung, damit die Zusammenarbeit gelingt, ist aus Sicht der Projektleiterin jedenfalls das Interesse an der Naturwissenschaft/Technik, das alle PartnerInnen teilen müssen, gegenseitiges Vertrauen und eine Offenheit v. a. von Seiten der PädagogInnen, sich auf Neues einzulassen.

\section{Wirkungen}

Die Kinder zeigten sich laut Projektleiterin begeistert. Insbesondere bei Kindern, die durch ihr Umfeld wenige Möglichkeiten vorfinden, solche Eindrücke zu sammeln, spürte sie nicht nur große Dankbarkeit, sondern glaubt auch, Perspektiven geöffnet zu haben. Lehrerlnnen zeigten sich in einem schriftlichen Feedback überrascht, wie konzentriert auch „schwierige“ Kinder mitarbeiteten und ruhigere Kinder beim Experimentieren aufblühten. 
Bei den Partnerlnnen scheint es zu einem Motivationsschub insbesondere der jungen ForscherInnen gekommen zu sein. Diese wollten alle im Projekt mitmachen. Auch konnten sich die Organisationen als engagierte Arbeitgeberlnnenbetriebe positionieren. Die FH erwartet sich durch die Mundpropanda eine Steigerung ihres Bekanntheitsgrades.

Die Stadt Tulln, bekannt als Blumen- und Messestadt, kann sich mit solchen Projekten als Forschungsstadt etablieren und die Vernetzung der Forschungseinrichtungen in der Region kann vorangetrieben werden. So lud die BOKU, Standort Tulln, die Technologykids zur alljährlichen Weihnachtsvorlesung ein und erste Weichen zur Einreichung einer Kinderuni im Sommer 2014, in der die BOKU, der Verein Technologykids und die Landesakademie Natur im Garten mitwirken werden, wurden gestellt.

\section{Lessons learnt}

Durch ein Talente regional Projekt werde der natürliche Wissensdrang der Kinder unterstützt und da diese zudem als MultiplikatorInnen in der Familie agieren, ist von einer Förderung des Interesses an Naturwissenschaft/Technik auf breiter Basis auszugehen. Die Programmlinie scheint absolut geeignet, die Kinder möglichst früh an solche Themenfelder heranzuführen.

Als Stärke werden die hohe Praxisorientierung gewertet sowie die zahlreichen Interaktionsmöglichkeiten von Forscherlnnen und Kindern. Voraussetzung ist die richtige Auswahl der Mitarbeitenden, denen ein solches Projekt ein persönliches Anliegen sein muss und die ihren Beruf lieben („,da springt dann der Funke auf die Kinder über") und die sehr experimentierfreudig und offen für Neues sind.

Eine neue Erfahrung stellt für die ForscherInnen die Arbeit mit den Kindern dar. Kindern in verschiedensten Altersgruppen adäquat zu begegnen und gleiche Themen altersgruppenspezifisch aufzubereiten, erweist sich als die pädagogische Herausforderung. Sehr aufwendig gestaltet sich auch die Koordination der zahlreichen PartnerInnen, die sich nicht in ausreichendem Maße im Budget abbilden lässt.

Die Programmlinie ist aber für die Projektleiterin und einige Partnerlnnen so attraktiv, dass sie derzeit wieder ein Projekt im Rahmen der zweiten Ausschreibungsrunde implementieren und für die dritte Ausschreibungsrunde eingereicht haben. 


\section{Fallstudie FEMtech Forschungsprojekte: FEMSMA}

\section{Projektteam}

Das FEMtech Forschungsprojekt FEMSMA (Langtitel: Automatisierte, gendersensible Verfahren zum Ausbau von Social Media Analysen als EDV-gestützte Forschungsmethodik) ist ein kooperatives Forschungsvorhaben welches im Themenfeld der Informations- und Kommunikationstechnologien angesiedelt ist. Das durchführende Konsortium setzt sich aus dem Unternehmen Inset Research \& Advisory Unternehmensberatungs $\mathrm{GmbH}$ (Projektleitung) sowie der außeruniversitären Forschungseinrichtung der Österreichischen Studiengesellschaft für Kybernetik (ÖFAl) zusammen. Der vorgesehene Projektzeitraum umspannt 24 Monate und läuft von Oktober 2012 bis September 2014. Dabei wird die Entwicklung eines Tools zur Genderbestimmung im Bereich der Social Media Analyse angestrebt.

\section{Ziele und Motivation}

Ziel des geförderten Projektes ist die Entwicklung eines automatisierten Verfahrens zu Genderbestimmung von Autorlnnen in Social Media Beiträgen, um diese in weiterer Folge für eine erweiterte Nutzung von Social Media Analysen (SMA) einsetzen zu können. Derzeit im Einsatz befindliche Instrumente für Social Media Analysen können der genderorientierten Bedarfsgerechtigkeit nicht vollständig Genüge tragen, da sie lediglich die Bereitstellung von genderneutralen Informationen zu den untersuchten Themenbereiche ermöglichen. Die zunehmende Bedeutung von SMA als Datenquelle (etwa in der Trendforschung), erfordert jedoch die präzise Bestimmung der Genderidentitäten, die anhand stilistischer Merkmale und Kontextfaktoren, automatisiert festgestellt werden sollen. Relevante Zielgruppen für die Nutzung der angestrebten Tools können dabei etwa in den Dienstleistungsbranchen der Markt- und Meinungsforschung sowie der Beratungstätigkeit angesiedelt sein.

Herangetragen an das Unternehmen wurde das betrachtete Innovationsvorhaben im Rahmen von FEMtech Forschungsprojekte von Seiten des Konsortialpartners. $\mathrm{Da}$ es bereits zu früheren Zeitpunkten gemeinsame Forschungstätigkeiten mit der betreffenden Studiengesellschaft gegeben hat und die Genderexpertise des Unternehmens der OFAI daher bekannt gewesen ist, wurde eine gemeinsame Implementierung und Umsetzung des FEMSMA-Projektes angestrebt.

Die Nutzung der Programmlinie FEMtech Forschungsprojekte ist darüber hinaus auf frühere Inanspruchnahmen von FFG Förderungen zurückzuführen. Zwar stellt dieses Projekt die erste Teilnahme des Unternehmens am Förderschwerpunkt Talente dar, jedoch führten die allgemein positiven Erfahrungen in Hinblick auf das Förderangebot der FFG, sowie projektrelevante Recherchetätigkeiten bezüglich geeigneter Förderangebote zu FEMtech Forschungsprojekte. 
Da der Förderschwerpunkt schließlich durch das Konsortium als sehr gut geeignet für den Projektkontext ausgemacht wurde, kam es in weiterer Folge zu einer Einreichung bzw. zur Teilnahme an dem Förderprogramm im Rahmen der ersten Ausschreibungsrunde.

\section{Projektumsetzung}

Zum Zeitpunkt des Projektantrages war die Unternehmensausrichtung stark auf den Bereich Forschung fokussiert, welcher allerdings in näherer Zukunft - auf Grund einer angestrebten Verlagerung hin zu verstärkten Consultingstätigkeit - wieder zurückgefahren werden soll. Im Rahmen der damals stark forschungsorientierten Ausrichtung des Unternehmens, wurde darüber hinaus die Zielsetzung der interdisziplinären Verknüpfung von IKT mit anschließenden Forschungsfeldern von hohem gesellschaftlichem Nutzen (etwa Migration, Integration, Sicherheit und Gesundheit) verfolgt.

Ein weiterer dieser interdisziplinären Schwerpunkte lag auf der Durchführung von Forschungsaktivtäten im IKT-Bereich an der Schnittstelle zu genderrelevanten Themenstellungen, welcher auch das FEMSMA Projekt zugeordnet werden kann. Dementsprechend war bereits ein ausreichender, unternehmensinterner Zugriff auf Genderexpertise für die Projektdurchführung vorhanden. Dies drückte sich einerseits in der allgemeinen Sensibilisierung bzw. den Projekterfahrungen der beteiligten ForscherInnen aus. Zum anderen konnte durch die Einbeziehung und Anstellung einer weiteren Gender-Expertin (aus dem Forschungsfeld der Genderlinguistik) in das Unternehmen, zusätzliches Know-how im Genderbereich gewonnen werden.

\section{Ergebnisse und Wirkungen}

Das Forschungsvorhaben befindet sich derzeit noch in der Umsetzungsphase, weshalb die Wirkungen und Projektresultate noch nicht bestimmt werden können. Unmittelbar lassen sich jedoch, neben den erwähnten Beschäftigungseffekten bzw. der damit verbundenen Ausweitung des Know-how Portfolios in genderrelevanten Themenbereichen, positive Auswirkungen auf die Unternehmenskompetenz im Bereich der Schnittstelle IKT und Gender feststellen. Die angestrebte Zielsetzung der verstärkten Verknüpfung der beiden Forschungsfelder und die Nutzung daraus resultierender Lerneffekte, konnte daher bereits während der Projektdurchführung als erreicht angesehen werden. In Hinblick auf die künftige, strategische Ausrichtung des Unternehmens ist jedoch unklar, in welchem Ausmaß der generierte Projektnutzen auch weiterhin zur Anwendung kommen kann. Auf gesellschaftlicher Ebene können positive Wirkungen anhand der erfolgten Projektdisseminationen im Zuge von Vorträgen und Publikationen definiert werden. 
Ohne die Förderung durch die FEMtech Forschungsprojekte wäre die Durchführung des Projektes allerdings nicht möglich gewesen, da der hohe Personalaufwand bzw. die daraus abgeleitete Kostenintensität durch das Konsortium nicht hätten finanziert werden können.

Eine weitere Projekteinreichung im Zuge von FEMtech Forschungsprojekte wäre, abhängig von der künftigen Unternehmensentwicklung, für das Unternehmen grundsätzlich vorstellbar, da es sich für dieses als transparentes und gut organsiertes Instrument herausgestellt hat. Darüber hinaus wird die Programmlinie als „einzige Chance zur umfassenden Abhandlung grundsätzlicher Genderaspekte und technologischer Fragestellungen" erachtet. Vor allem für die Abwicklung von innovativen Forschungsprojekten mit wesentlichem gesellschaftlichem Nutzen erweist sich FEMtech Forschungsprojekte nach Einschätzung des Unternehmens daher als sehr geeignetes Förderinstrument. 
Fallstudie FEMtech Forschungsprojekte: living gender

\section{Projektteam}

Das Konsortium des living_gender Kooperationsprojekts zur Entwicklung von Planungsinstrumenten für gender- und diversitätsgerechten nachhaltigen Wohnbau setzt sich aus drei ProjektteilnehmerInnen zusammen. Zwei davon sind den auBeruniversitären Forschungseinrichtungen (Österreichisches Ökologie Institut und Österreichische Gesellschaft für Umwelt und Technik, ÖGUT) sowie einer dem Bereich der Unternehmenspartnerlnnen (Schöberl \& Pöll GmbH) zuzuordnen. Die Projektleitung des FEMtech Forschungsprojektes obliegt dem Österreichischen Ökologie Institut. Die Laufzeit des geförderten Forschungsvorhabens beträgt zwei Jahre und erstreckt sich über den Zeitraum Juni 2012 bis Mai 2014.

\section{Ziele und Motivation}

Im Vordergrund des Projektes steht die Berücksichtigung unterschiedlicher Lebenssituationen und Lebensphasen von verschiedensten Personengruppen in der Planung und Gestaltung von hochenergieeffizienten Wohngebäuden. Die dadurch angestrebte Gender- und Diversitätsgerechtigkeit im Wohnbau schafft einerseits die Möglichkeit der gesteigerten Selbstbestimmung in divergierenden Lebenslagen, sowie andererseits die nachhaltige und langfristige Nutzbarkeit von kosteneffizienten Wohnräumen für die Zukunft. Dabei werden abseits der Genderperspektive auch Aspekte wie das Alter, Behinderungen, Migrationshintergrund oder Einkommen in die Betrachtungen einbezogen. Das FEMtech Forschungsprojekt hat dementsprechend folgende konkrete Zielsetzungen:

- Zusammenführung von gender- und diversitätsrelevantem Wissen und ökologischen Standards im Wohnbau,

- Schaffung von Grundlagen für die technische Umsetzbarkeit des detaillierten Kriterienkatalogs,

- Initiierung einer Umsetzung in die Breite.

Die Idee zur Implementierung des living_gender Forschungsprojektes war seitens der involvierten Forschungsinstitute bereits länger vorhanden. Auf Grund der fehlenden bzw. ungeeigneten Fördermöglichkeiten zur Finanzierung von Forschungsprojekten im Untersuchungsfeld Wohnbau und Diversität bzw. Gender, konnte diese jedoch nicht umgesetzt werden. Erst im Zuge der ersten Ausschreibung der themenoffenen FEMtech Forschungsprojekte bot sich die Möglichkeit, das genderrelevante und technikorientierte Vorhaben tatsächlich zu realisieren. Die Möglichkeit der ausdrücklichen Betrachtung von Diversität ermöglicht es dem Konsortium, sich umfassende Kompetenzen anzueignen, die mit anschließenden Themenfeldern verknüpft werden können. Darunter fällt etwa der Bereich der Gebäudebeurteilung. 


\section{Projektumsetzung}

Genderexpertise bzw. Erfahrungen bezüglich der Umsetzung von geförderten Forschungsprojekten mit genderrelevanten Inhalten, sind auf Seiten der Fördernehmerlnnen auf Grund vorangegangener Projekte vorhanden. Als Anzeichen für die allgemeine Sensibilisierung für dieses Thema kann darüber hinaus die wiederholte Teilnahme am Förderschwerpunkt Talente bzw. an FEMtech angeführt werden.

Gegenüber den vergleichbaren Forschungsvorhaben der ersten Ausschreibung zu FEMtech Forschungsprojekte, zeigt sich im Zuge des living_gender Projektes die ganzheitliche Betrachtung des Diversitätsaspekts über die vorrangige Berücksichtigung des Genderbegriffes hinweg. Dies ist seitens des Projektkonsortiums auf die vorgelagerte Notwendigkeit von Diversitätsgerechtigkeit zurückzuführen, um in weiterer Folge auch die Gendergerechtigkeit gewährleisten zu können. Da das Projekt die Erfassung von veränderten Bedürfnissen im Wohnbau auf der gesamten gesellschaftlichen Ebene anstrebt, entspräche die ausschließliche Betrachtung des Genderaspektes nur die Erfassung eines relevanten Teilbereiches. Einflüsse weiterer Charakteristika (etwa Gender und Alter, Gender und Migration) könnten dann nur ungenügend einbezogen werden. Daher orientieren sich Fragestellungen, die aus der Projektumsetzung entstehen, zwar deutlich stärker in Richtung Diversität, allerdings läuft die notwendige Beurteilung und Einbeziehung des Genderaspekt gleichzeitig mit („die Genderrelevanz zieht sich wie ein roter Faden durch die Projektdurchführung und wird immer parallel zur Diversitätsgerechtigkeit betrachtet").

\section{Ergebnisse und Wirkungen}

Erkennbare Wirkungen des Projektes auf die beteiligten Fördernehmerlnnen sind, trotz des laufenden Projektstatus, seitens der Fördernehmerlnnen bereits erkennbar. Darunter fällt etwa die bereits zu Projektbeginn erforderliche, konsortiumsinterne Sensibilisierung und Definition eines übereinstimmenden ArbeitsVerständnisses. Dieses ist für die kongruente Definition der diversitäts- und gendergerechten Anforderungen in Bezug auf das Themenfeld des nachhaltigen Wohnbaus erforderlich gewesen.

Während die Einbeziehung des Diversitäts- und Genderaspekts im Wohnbau in den vergangenen Jahren lediglich als Randthema wahrgenommen wurde, kann es mittlerweile Fuß fassen, wobei auch das living_gender Projekt zur gesteigerten Wahrnehmung dieses Forschungsbereiches beitragen kann. In weiterer Folge kommt es auf Grund des gestiegenen Interesses an dem Forschungsfeld zu einer fortlaufenden Know-how Intensivierung innerhalb des Themas Wohnbau. Diese Entwicklung konnte, anhand der angestrebten Projektzielsetzung und der bisher gewonnenen Erkenntnisse in Bezug auf diversitätsgerechtes Wohnen, mitgetragen werden. 
Durch die Integration der gender- und diversitätsrelevanten Aspekte konnten relevante Bedürfnisse definiert bzw. adressiert und in den Bereich des nachhaltigen Wohnbaus eingeführt werden (Leitfaden für die Umsetzung von gender- und diversitätsgerechtem Wohnbau). Die daraus gewonnenen Ergebnisse sind in weiterer Folge für die Wohnbauplanung von hoher Relevanz und können darüber hinaus für die künftige Umsetzung von gender- und diversitätsgerechten Wohnbauten eingesetzt werden. Mehr noch haben die erzielten bzw. angestrebten Ergebnisse das Potenzial, auch in weiteren Anwendungsfeldern bzw. Themenbereichen angewandt werden zu können. 


\section{Fallstudie FEMtech Forschungsprojekte: Mobility4Job}

\section{Projektteam}

Das Mobility4Job Forschungsprojekt ist ein Kooperationsvorhaben welches federführend vom Institut für Verkehrswesen der Universität für Bodenkultur abgewickelt wird. Gemeinsam mit zwei UnternehmenspartnerInnen (Büro für nachhaltige Kompetenz B-NK GmbH, Rosinak \& Partner ZT GmbH) und einer wissenschaftlichen Partnerin (Kammer für Arbeiter und Angestellte Wien) wird im Zuge der Projektdurchführung von Juli 2012 bis September 2014 die Identifikation mobilitätsbedingter Hemmnisse für den Einstieg in die Erwerbstätigkeit angestrebt und nach nachhaltigen, innovativen Mobilitätslösungen gesucht.

\section{Ziele und Motivation}

Mobilität im ländlichen Raum ist auf Grund der geringen Arbeitsplatz- und Versorgungsdichte sowie mangels umfassender öffentlicher Verkehrsangebote stark vom Individualverkehr geprägt. Dabei ist der private Pkw das wesentliche Instrument zur Erfüllung vorhandener Versorgungs- und Erwerbspflichten. Steht einem Haushalt kein bzw. nur ein Auto zur Verfügung, kann es zu einer eingeschränkten Mobilität der verbleibenden Haushaltsmitglieder kommen. Diese Pkw-Abhängigkeit drückt sich im Aufkommen von sozialen, ökonomischen und ökologischen Problemen aus, wobei die Mobilitätshemmnisse erwiesenermaßen in geschlechterspezifisch unterschiedlichen Weisen wirken können.

Die damit in Zusammenhang stehende Zielsetzung des Mobility4Job Projektes ist es daher,

- mobilitätsbedingte Hemmnisse und Einschränkungen für die Erwerbstätigkeit zu identifizieren und

- Mobilitätsvoraussetzungen und -dienstleistungen zu definieren, die für eine chancengerechte Teilhabe an der Erwerbs-, Familien-, und Versorgungsarbeit im ländlichen Raum notwendig sind.

Zur Identifizierung und Definition der erwähnten Problemstellungen bzw. Lösungsansätze, wurde ein Untersuchungsgebiet im südlichen Niederösterreich ausgewählt, welches insgesamt 30 Gemeinden und rd. 60.000 Einwohnerlnnen umfasst. Dabei wurde die Bevölkerung zur notwendigen Datenerfassung im Zuge von unterschiedlichen Partizipationsprozessen in die Projektdurchführung eingebunden. 


\section{Projektumsetzung}

Der Grundgedanke zur Umsetzung dieses genderrelevanten Forschungsprojektes im Mobilitätsbereich ist im Rahmen fachlicher Diskussionen am Rande eines Projektmeetings während eines IV2S-Infrastrukturprojekt ${ }^{32}$ entstanden. In Folge dieser Diskussion ist man über die hohe gesellschaftliche Relevanz übereinkommen und die bisher eingeschränkte Berücksichtigung des Genderthemas in Mobilitätsfragen gestoßen, woraus sich die Idee der Implementierung dieses FEMtechForschungsprojekts ergeben hat. Der Gender-Aspekt war dementsprechend der initiale Gedanke des Forschungsvorhabens und ist von Beginn an in der Umsetzung des Projektes verankert gewesen.

Die Verwendung des spezifischen Förderinstrumentes FEMtech-Forschungsprojekte ergab sich dabei allerdings eher zufällig. Die Suche nach einem geeigneten Förderprogramm war bereits angelaufen, als man durch Erhalt des FFGNewsletters auf das Förderangebot aufmerksam geworden ist und sofort die Gelegenheit ergriffen hat sich im Rahmen der ersten Ausschreibung dafür zu bewerben. Zwar wurden in der Vergangenheit bereits Förderangebote der FFG in Anspruch genommen, die Suche nach einem passenden Förderangebot gestaltete sich jedoch trotzdem als schwierig, da die sozialwissenschaftliche Ausrichtung des Forschungsprojektes von den üblichen, rein technologieorientieren Forschungsfeldern des Instituts abgewichen ist.

Die Partnerlnnen, die zur Projektdurchführung hinzugezogen wurden, waren bereits aus vorhergehenden Kooperationen bekannt und wiesen zusätzliche Expertise sowohl in sozialwissenschaftlichen Fragestellungen als auch in Hinblick auf die notwendigen Genderkompetenzen auf. Darunter fallen etwa die projektrelevante, praxisorientierte Umsetzung von Teilnahmeprozessen oder die Einbindung von Stakeholdern in umfassende Partizipationsvorhaben.

\section{Ergebnisse und Wirkungen}

Da das Forschungsvorhaben noch nicht abgeschlossen ist, können vor allem die projektbedingten Ergebnisse und Outcomes noch nicht bestimmt werden. Jedoch lassen sich bereits Wirkungen bei den beteiligten Forschungseinrichtungen und Unternehmen feststellen. Diese drücken sich etwa in Lerneffekten auf persönlicher und Organisationsebene aus, die auf Grund der Projektstrukturierung und der Charakteristik der Ergebnisse auch auf die partizipierende Bevölkerung bzw. eingebundenen AkteurInnen, wirken.

Neben dem Aufbau von genderrelevante Wissensgrundlagen in dem betreffenden Forschungsbereich hat etwa die Zusammenarbeit mit unterschiedlichen Stakeholdern und Organisationen zu einer Bewusstseinsschärfung dahingehend geführt,

32 Förderprogramm im Bereich intelligenter Verkehrssysteme und Services 
dass die Interpretation von Gender mehrdimensional erfolgt. Unterschiedliche Lebensentwürfe und Mobilitätsbedürfnisse bestimmen die Frage des chancengerechten Zuganges zum Erwerbs- und sozialen Leben sowie dem Mobilitätszugang. Dabei konnten überraschende Ansatzpunkte festgestellt werden, die wechselwirkende Einschränkungen in der Mobilität bewirken (z.B. Wirkungen von Mobilitätshindernissen der Kinder auf die Betreuenden).

So ist etwa auf Grund des unterschiedlichen Verständnisses des Genderbegriffes eine dementsprechende Berücksichtigung in Hinblick auf die Zusammenarbeit mit verschiedensten Stakeholdern notwendig, um das Mobilitätsthema ins Zentrum der Betrachtungen rücken zu können.

Für die Bevölkerung und die eingebundenen Unternehmen (u.a. VOR, AMS) hat sich aus dem Projekt eine Sensibilisierung bzw. verstärkte Beschäftigung mit dem Begriff Gender ergeben. Auf Grund der starken Beteiligung der Zielgruppen an dem Projekt kann dabei sowohl von einer gesteigerten Wahrnehmung des Genderthemas (neue Betrachtungsweisen) als auch von einer durchaus hohen Bereitschaft, an der Lösung genderrelevanter Problemstellungen mitzuarbeiten, gesprochen werden. Des Weiteren hat sich der hohe Stellenwert eines unabhängigen Mobilitätszuganges für die Organisation des Alltags offenbart, vor allem für Personen mit Betreuungspflichten.

Darüber hinaus konnte durch die Projektumsetzung und die gewonnene Genderkompetenz des gesamten Konsortiums, eine Nische im Forschungsfeld der Mobilität besetzt werden. Der durch das Projekt angestrebte und auch erfolgte Wissensaufbau, kann darüber hinaus die Möglichkeit bieten, einen längerfristigen Forschungs- und Begleitprozess in der Untersuchungsregion zu implementieren. Allerdings wäre dieser fortlaufende Prozess wiederum mit der Verfügbarkeit geeigneter und ausreichender Fördermittel verknüpft. 


\section{Fallstudie FEMtech Karriere: Diversity@RBOS}

\section{Organisation}

Die Robert Bosch AG ist eine von sieben Gesellschaften und das Headquarter der Bosch-Gruppe in Österreich. Die Bosch-Gruppe ist ein internationales Technologie- und Dienstleistungsunternehmen mit den Geschäftsbereichen Kraftfahrzeugtechnik, Gebrauchsgüter, Industrietechnik sowie Energie- und Gebäudetechnik. In Österreich beschäftigt die Bosch-Gruppe fast 3.000 Personen (über 280.000 MitarbeiterInnen weltweit) und hat 13 Standorte.

\section{Ziele und Motivation}

Die Bosch-Gruppe hat sich der Vielfalt (Diversity) verschrieben und richtet ihren Fokus dabei auf die vier Dimensionen Geschlecht, Generation, Internationalität und Arbeitskultur. In Österreich wurde das Thema Gender als Schwerpunkt herausgegriffen. Dabei wurden folgende Ziele formuliert:

- Der Anteil der weiblichen Führungskräfte sollte deutlich erhöht werden, wobei zwischen drei Einkommensstufen (Salary Level - SL) unterschieden wurde.

- Der Anteil der Technikerinnen sollte erhöht werden. Noch ambitioniertere Ziele wurden für 2015 bzw. 2020 gesetzt.

- Bosch sollte als attraktiver Arbeitgeber positioniert werden.

Die Personalverantwortliche plante Maßnahmen und stieß über Mundpropaganda zu FEMtech Karriere, dessen Förderung den strukturierten Aufbau ineinandergreifender Maßnahmen und eine systematische Bearbeitung des Themas erlaubte.

\section{Projektinhalte}

Im Arbeitspaket "get organized“ erfolgte neben dem Projektmanagement v. a. die Analyse und Priorisierung der Zielausbildungstätten, d.h. Schulen, mit denen eine stärkere Vernetzung erfolgen sollte, um frühzeitig mit potentiellen Nachwuchskräften in Kontakt treten zu können. In einem Auftaktworkshop mit einer externen Diversityexpertin, den Diversity KoordinatorInnen und der Projektverantwortlichen erfolgte eine Zielabklärung und die Planung der Aktivitäten. Die Personalleiterin berichtete in den Managementteam-Sitzungen regelmäßig über das Projekt.

Das Arbeitspaket „Diversity@RBOS“ war dem Aufbau der Genderkompetenz gewidmet. In internen Workshops wurde die Relevanz des Genderthemas für das Unternehmen thematisiert. Außerdem wurden die HR-MitarbeiterInnen in internen Terminen, den sogenannten „Wissenstankstellen“ zum Thema Diversity gebrieft. 
„Communicate about Diversity@RBOS“ fasste die Maßnahmen im Bereich Öffentlichkeitsarbeit zusammen. Neben der Neugestaltung von Flyern, Plakaten und der Platzierung von Artikeln in der MitarbeiterInnenzeitung wurde besonderes Augenmerk auf den Internetauftritt gelegt. Es erfolgte eine Anpassung der Karrierehomepage („,bosch-career.at"), wobei z. B. bei der Stellenbörse nun offene Stellen in weiblicher Form mit dem Verweis w/m ausgeschrieben werden. Über Facebook sollen verstärkt Schülerinnen angesprochen werden.

Im Arbeitspaket „Promote Diversity@RBOS; Get started to connect with the future“ stand das Personalmanagement und -marketing im Mittelpunkt. Dabei galt es im Unternehmen tätige Technikerinnen verstärkt als Role Models bei (Karriere)Messen, Betriebsbesichtigungen etc. zu zeigen. Im „Karriere-Standard“ wurden in einer Sonderausgabe auf das Projekt verwiesen und Role Models aufgezeigt. Zudem wurde die Bildungspartnerschaft zwischen dem Unternehmen und verschiedenen HTLs intensiviert.

Die interne Kommunikation erfolgte $u$. a. über eine Informationsveranstaltung aller Mitarbeiterlnnen durch den Vorstand und die Sichtbarmachung des DiversityLogos im Headquarter.

Im Arbeitspaket „New FEmALE Employees“ wurden Führungskräfte gezielt in Hinblick auf weibliche Bewerberinnen und den Umgang mit innen in Bewerbungssituationen sensibilisiert. Das Arbeitspaket "Get moving“ stand im Zeichen von Weiterbildung und Schulungsmaßnahmen. So fand z. B. ein Führungskräfte-Workshop zum Thema „Machtspiele zwischen Männern und Frauen im alltäglichen Arbeitsumfeld" statt und Netzwerktreffen für weibliche Führungskräfte und Potentialträgerinnen wurden organisiert, aus denen die Gruppe „Women@Bosch“ entstand. Zudem gab es mehrere Seminare für weibliche Führungskräfte.

\section{Ergebnisse und Wirkungen}

Die Maßnahmen im Rahmen des FEMtech Karriere Projektes zeigen Wirkung, die sich auch in Zahlen widerspiegeln. Der Anteil weiblicher Technikerinnen unter den Neueintritten bei der Robert Bosch AG ist stark gestiegen. Die Ziele im Bereich der weiblichen Führungskräfte konnten (noch) nicht erreicht werden, der Anteil an weiblichen PraktikantInnen hat sich dagegen erhöht. Durch die verstärkte Präsenz weiblicher Role Models gelingt es dem Unternehmen zunehmend, sich als attraktiver Arbeitgeber auch für Frauen zu positionieren.

Der Umdenkprozess macht sich auch im Bereich Vereinbarkeit von Beruf und Familie bemerkbar. Gingen 2012 erst vier Männer in Väterkarenz, waren es 2013 schon 16. Insgesamt scheint das Thema Diversity im Allgemeinen und Gender im Besonderen nun besser im Unternehmen verankert. 


\section{Lessons learnt}

Trotz vieler Erfolge im Projekt und der verstärkten Sensibilisierung und Verankerung der Leitgedanken erkannten die Projektverantwortlichen, dass solch umfassende Veränderungen Zeit brauchen. V. a. in Hinblick auf die Erhöhung des Anteils der weiblichen Führungskräfte erwiesen sich die Ziele als zu ambitioniert. Da aber der Anteil der Technikerinnen stark ansteigt und wachsendes Interesse der Mädchen an Praktika zu beobachten ist, sollte es dem Unternehmen in Folge auch gelingen, den Anteil der Frauen in Führungspositionen entsprechend zu steigern.

Durch die Förderung konnte das Thema prominenter platziert werden und ging nicht im Betriebsalltag unter, was wiederum zu einer verstärkten Veränderung des Mindsets im Unternehmen beigetragen hat. Das Projekt wird als Anreiz verstanden, die Themen Gender und Diversity im Unternehmen weiter zu verfolgen, da ersichtlich wurde, dass eine nachhaltige Veränderung kontinuierlicher Anstrengungen und Zeit bedarf. Daher ist seitens der Robert Bosch AG geplant, wieder bei FEMtech Karriere mit anderer Schwerpunktsetzung einzureichen. Zudem bietet das Unternehmen auch SchülerInnenpraktika und FEMtech Praktika für Studentinnen an. 


\section{Fallstudie FEMtech Karriere: Pro-GRESS}

\section{Organisation}

VIRTUAL VEHICLE ist ein in Graz angesiedeltes Kompetenzzentrum mit 200 MitarbeiterInnen, das sich der anwendungsnahen Fahrzeugentwicklung und zukünftigen Fahrzeugkonzepten für Straße und Schiene widmet. Das Zentrum umfasst mittlerweile mehr als 90 IndustriepartnerInnen und neben der TU Graz, die das wissenschaftliche Rückgrat bildet, mehr als 30 wissenschaftliche PartnerInnen. VIRTUAL VEHICLE ist außerdem Trägergesellschaft des COMET K2Forschungsprogramms „K2-Mobility - Sustainable Vehicle Technologies“ (20132017).

\section{Ziele und Motivation}

Ziel des von VIRTUAL VEHICLE durchgeführten FEMtech Karriere Projekts ist es, bestmögliche Arbeitsbedingungen unabhängig vom Geschlecht zu bieten und Chancengleichheit weiterzuentwickeln. Es sollen

- die Genderkompetenz erweitert werden,

- eine Werbe- und Personalmarketingstrategie erarbeitet werden, die beide Geschlechter gleichermaßen anspricht und

- Maßnahmen der betriebliche Gesundheitsförderung unter Genderaspekten umgesetzt werden.

Das Kompetenzzentrum hat in seinen Zielsetzungen die Nachwuchspflege verankert und ist sich der Wichtigkeit der Sicherung von Humanressourcen bewusst. Zudem gibt es Auflagen von der FFG, den Frauenanteil im technischen Bereich zu steigern und Gender Mainstreaming zu implementieren (hier muss auch regelmäßig berichtet werden). Dennoch können Personalentwicklungsprojekte schwer ohne Förderung durchgeführt werden, da die Personalverantwortliche das Budget für derartige Maßnahmen akquirieren muss („Was nicht auf Projekte gebucht werden kann, ist ein Problem"). Daher gehen auch bereits etablierte Frauenfördermaßnahmen, wie das Frauennetzwerk oder ein Recruitingfolder für Frauen, auf ein Vorgängerprojekt (2009 bis 2011) zurück, das den Schwerpunkt auf die KarriereFörderung von Mitarbeiterinnen legte. Diese Bemühungen sollten im Rahmen des FEMtech Karriere Projekts fortgesetzt und vertieft werden. Zudem ist die Projektleiterin Mitglied eines Netzwerks für gleichstellungsorientiertes Personalmanagement in Graz und Mitbegründerin eines Projektleiterinnennetzwerks.

\section{Projektinhalte}

Zum Aufbau der Genderkompetenz wurde eine Genderbeauftragte, die zuvor eine entsprechende Ausbildung und ein Coaching erhalten hat, etabliert und in einer MitarbeiterInnenveranstaltung vorgestellt. Zudem wurde ein Gendertraining für (Nachwuchs-)Führungskräfte durchgeführt. Dabei war es schwierig, die wissen- 
schaftlichen Bereichsleiter, die nicht am Kompetenzzentrum angestellt sind, zu erreichen und einzubinden.

Im Bereich Öffentlichkeitsarbeit wurde ein neuer Messestand entworfen, der die Werte des Unternehmens in Form einer Wordcloud darstellt. Diese wurden im Rahmen von Workshops mit MitarbeiterInnen erarbeitet. So sollten die im Unternehmen geschaffenen besseren Rahmenbedingungen für Frauen und Männer nach außen getragen werden, um das Kompetenzzentrum als attraktiven Arbeitgeber zu positionieren. Zudem wird ein Video über Praktikantinnen für die Homepage erstellt.

In Hinblick auf die Work Life Balance wurde ein Gesundheitsförderungsprojekt initiiert. Dabei sollte die Gesundheitsförderung unter Genderaspekten neu gestaltet und verbessert werden. Es erfolgte eine MitarbeiterInnenbefragung, die die Belastungen im Arbeitsalltag und spezifische Bedürfnisse von Frauen identifizieren sollte. In der Folge wurden Gesundheitszirkel gegründet, die mit externer Hilfe Maßnahmen zur Gesundheitsförderung abgeleitet haben. Daraus entstanden u. a. ein Ernährungsworkshop, ein Anti-Stress-Workshop, Entspannungsübungen für Arbeitspausen und Wirbelsäulengymnastik. Bei diesen Veranstaltungen war die Teilnahme von Frauen überproportional. Eine zweite Mitarbeiterlnnenbefragung dient der Evaluierung der Maßnahmen, die in der Folge sicherlich weitergeführt werden. ${ }^{33}$

\section{Ergebnisse und Wirkungen}

Beim Management von VIRTUAL VEHICLE konnte nicht zuletzt durch die Akquirierung der Fördermittel für das FEMtech Karriere Projekt, die die Wichtigkeit des Themas unterstreicht, ein Umdenken bewirkt werden. Waren früher Sätze wie „es gibt halt keine Frauen in der Technik" an der Tagesordnung, sind diese kaum mehr zu hören. Die Gendertrainings erhöhten die Sensibilität der Führungskräfte in Hinblick darauf, wie Unterschiede zwischen den Geschlechtern wahrgenommen werden können und wie damit in Hinblick auf Motivation und Feedbackkultur umgegangen werden kann. Inzwischen kam es auch zur Implementierung der ersten Gruppenleiterin, was sicherlich auch teilweise auf den im Projekt initiierten Umdenkprozess zurückzuführen ist.

Der Frauenanteil im technischen Bereich hat sich bei VIRTUAL VEHICLE zwischen Ende 2012 und Ende 2013 von 11 \% auf 14 \% erhöht und die HR-Verantwortliche kann beobachten, dass es nun wesentlich mehr Bewerberinnen gibt. Studentinnen erklären, dass sie sich vom Kompetenzzentrum angesprochen und eingeladen fühlen. Auch die Fluktuationsrate konnte durch die Personalentwicklungsmaßnahmen, die zum Großteil im Projekt entstanden sind, von $10 \%$ auf $5 \%$ gesenkt wer-

33 Zum Zeitpunkt des Interviews war die Befragung noch nicht abgeschlossen, ebenso wie das FEMtech Karriere-Projekt. 
den. Die Väterkarenz scheint zum Standard zu werden, die meisten Väter nehmen zumindest 2 Monate davon in Anspruch.

Einen positiven Nebeneffekt brachte zudem die Installierung der Genderbeauftragten. Diese wird nun vermehrt bei Projektanträgen hinzugezogen, bei denen die Genderrelevanz und die Zusammensetzung des Forschungsteams argumentiert werden muss. Führungskräfte und ProjektleiterInnen schätzen diese im Haus verfügbare Kompetenz sehr.

\section{Lessons learnt}

Obwohl die Personalentwicklung und Nachwuchspflege im Allgemeinen und die Frauenförderung im Besonderen in einem Kompetenzzentrum grundsätzlich auf der Agenda steht, können konkrete Maßnahmen kaum ohne zusätzliche Fördermittel umgesetzt werden. Diese verleihen dem Thema außerdem intern zusätzliche Wichtigkeit. In der Forschungseinrichtung ist man sich bewusst, dass Änderungen nicht von heute auf morgen erfolgen können, aber davon überzeugt, dass ein solches Projekt mittel- bis langfristig starke Wirkungen hervorrufen kann. Dafür spricht die breite positive Resonanz auf die Initiative. So empfiehlt die HR-Verantwortliche die Programmlinie auch regelmäßig anderen HR-ManagerInnen weiter. Zudem bietet das Kompetenzzentrum neben dem FEMtech Karriereprojekt auch FEMtech Praktika für Studentinnen und ist in Talente regional-Projekte involviert. 


\section{Fallstudie FEMtech Karriere: Wogoto}

\section{Organisation}

Im-plan-tat, Reinberg und Partner OG, ist ein technisches Büro für Raumplanung mit Sitz in Krems/Donau und einer Zweigstelle in Tulln (Niederösterreich). Im Unternehmen sind insgesamt 10 Personen beschäftigt, vier Partnerlnnen (davon eine Frau) und sechs Mitarbeiterlnnen (davon fünf Frauen). Das Leistungsspektrum umfasst Raum- und Standortplanung, Ressourcen- und Umweltmanagement, Infrastrukturplanung sowie Freizeitinfrastruktur und Tourismus, wobei das Unternehmen bis dato v. a. in Niederösterreich tätig ist.

\section{Ziele und Motivation}

Das Unternehmen war durch eine sehr flache Hierarchie gekennzeichnet und einer der Geschäftsführer wollte das Team neu strukturieren und nachhaltig ans Unternehmen binden. Über die Aussendungen der FFG (das Unternehmen hat viel Erfahrung mit der Forschungsförderung) wurde er auf FEMtech Karriere aufmerksam und erkannte, dass die Programmlinie sehr geeignet für die spezifischen Herausforderungen im Unternehmen war. Da das Team vorwiegend aus jungen Frauen besteht, galt es zum einen das Arbeitsleben und den Arbeitsalltag von Frauen in technischen Berufen und die damit verbundene Problematik und zum anderen das Thema Vereinbarkeit von Beruf und Familie verstärkt zu adressieren. Mit der Umsetzung dieser Vorhaben im Rahmen des FEMtech Projekts Wogoto konnten die Personalentwicklungspläne systematisch und wesentlich breiter umgesetzt und eine intensive Auseinandersetzung mit der Genderthematik ermöglicht werden.

Das Projekt hatte folgende Ziele:

- Das Team für das Thema Gender zu sensibilisieren.

- Eine potenzielle Teamleiterin aus dem Team zu identifizieren und als stellvertretende Geschäftsführerin zu etablieren.

- Eine bessere Work-Life-Balance zu ermöglichen.

- Die Mitarbeiterinnen im männlich dominierten Umfeld zu stärken.

\section{Projektinhalte}

Das Projekt wurde mit externer Hilfe eines Coachs konzipiert, wobei sich das gesamte Team einbringen und zu behandelnde Themen vorschlagen konnte.

Zum Aufbau der Genderkompetenz wurden zunächst zwei Workshops zum Thema Gender Mainstreaming (allgemein und im Berufsfeld Raumplanung / Landschaftsplanung im Besonderen) getrennt für die Geschäftsführung und die Mitarbeiterinnen abgehalten. Im Anschluss fanden gemeinsame Workshops zu den Themen Berufsalltag von Technikerinnen in einem männlich dominierten Berufsfeld und Gender in der täglichen Planungsarbeit statt. 
Zur Identifikation einer Stellvertreterin setzte sich das Team in einem ganztätigen Workshop mit Schlittenhunden mit den eigenen Führungsqualitäten auseinander; die gewonnenen Eindrücke wurden in einem Reflexionsworkshop analysiert. Dabei kristallisierten sich zwei Personen für eine zukünftige Führungsposition heraus.

Im Bereich der Work-Life-Balance wurde ein Workshop zur Heranführung an das Thema sowie zur Burnout-Prävention durchgeführt. In einem zweiten Workshop galt es, das derzeitige Arbeitszeitmodell $z u$ analysieren und gegebenenfalls $z u$ adaptieren. Dabei kam es zu einer weiteren Flexibilisierung der Arbeitszeiten. Ein dritter Workshop befasste sich mit dem Thema Berufs- und Familienplanung, der durch die Schwangerschaft der Projektleiterin besondere Aktualität erlangte. Hierbei wurden der Einstieg nach der Karenz und der Wegfall von Führungskompetenz diskutiert.

Zum Thema Karriereentwicklung wurden allen Mitarbeiterinnen zwei Einzelcoachings angeboten sowie Mitarbeiterinnengespräche zur individuellen Weiterentwicklung geführt. Alle Mitarbeiterinnen nahmen in der Folge an Weiterbildungsmaßnahmen teil.

In Folge des Projekts kam es auch zu einer Überarbeitung der Firmenhomepage. Diese wurde gendersensibel gestaltet und das FEMtech Projekt wurde präsentiert. An der HAK Tulln wurde darüber hinaus in einem Workshop mit PädagogInnen und SchülerInnen das Berufsfeld Raum-, Energie- und Verkehrsplanung präsentiert und gezeigt, dass Technikerinnen in einem männlich dominierten Berufsfeld Erfolg haben können. In der Folge wurde eine SchülerInnengruppe (zwei Schülerinnen und ein Schüler mit Migrationshintergrund) bei Ihrer Maturaarbeit unterstützt.

In einem abschließenden Workshop wurde das Projekt reflektiert, ein gemeinsames Kunstwerk gestaltet und zukünftige Herausforderungen für das Unternehmen diskutiert.

\section{Ergebnisse und Wirkungen}

In Folge des von im-plan-tat durchgeführten FEMtech Karriere Projekts wurde eine Mitarbeiterin als Teamleiterin und stellvertretende Geschäftsführerin eingesetzt. Eine zweite zurzeit in Karenz befindliche Mitarbeiterin wurde als mögliche Führungskraft identifiziert. Die Organisation zeigt sich laut Geschäftsführung damit personell breiter und stabiler aufgestellt.

Ein sehr flexibles Arbeitszeitmodell wurde installiert. Dieses sieht zwei fixe Bürotage (die jedoch bei Bedarf verschoben werden können) vor, die sonstige Arbeitszeit ist frei einzuteilen (vorwiegend Telearbeit). Zudem werden bei der Studentin im Team Ferien und studienintensivere Zeiten bei der Zeiteinteilung verstärkt berücksichtigt. Ein Modell für einen zeitnahen Einstieg nach der Karenz wird erprobt. 
Das gesamte Team zeigt sich hinsichtlich der Genderthematik sensibilisiert, kennt die damit verbundene Problematik und kann sich auf bestimmte Situationen entsprechend vorbereiten bzw. in diesen besser reagieren (z.B. „Vorbehalte von Bürgermeistern können besser pariert werden"). Die Mitarbeiterinnen sind gestärkt und selbstbewusst in ihrer Rolle als Technikerinnen. Die Teamzusammengehörigkeit wurde gestärkt.

\section{Lessons learnt}

Obwohl alle gesetzten Ziele erreicht wurden, ist sich das Team von im-plan-tat bewusst, dass mit dem Projektende das Thema nicht von der Agenda verschwindet. Im Gegenteil zeigen sich die Genderthematik und die Work-Life-Balance dauerhaft präsent und es scheint klar, dass nun getroffene Vereinbarungen vor dem Hintergrund geänderter Rahmenbedingungen immer wieder hinterfragt und angepasst werden müssen.

FEMtech Karriere ermöglichte dem Unternehmen, Themen und Inhalte detailliert zu bearbeiten, die sonst im Arbeitsalltag untergingen. Insbesondere im-plan-tat als Kleinunternehmen mit beschränkten finanziellen Ressourcen wäre ohne die Förderung nicht in der Lage ein solches Projekt zu implementieren, obwohl die Notwendigkeit v. a. im technischen Bereich in hohem Maße gegeben ist. Daher scheint auch die Einreichung eines ähnlichen, weiterentwickelten Projekts vor dem Hintergrund sich ändernder Teamkonstellationen nicht unwahrscheinlich. 


\subsection{Zusätzliche Grafiken}

\section{Praktika für Schülerlnnen}

\section{Grafik 12 Verteilung der SchülerInnenpraktika nach Schultypen}

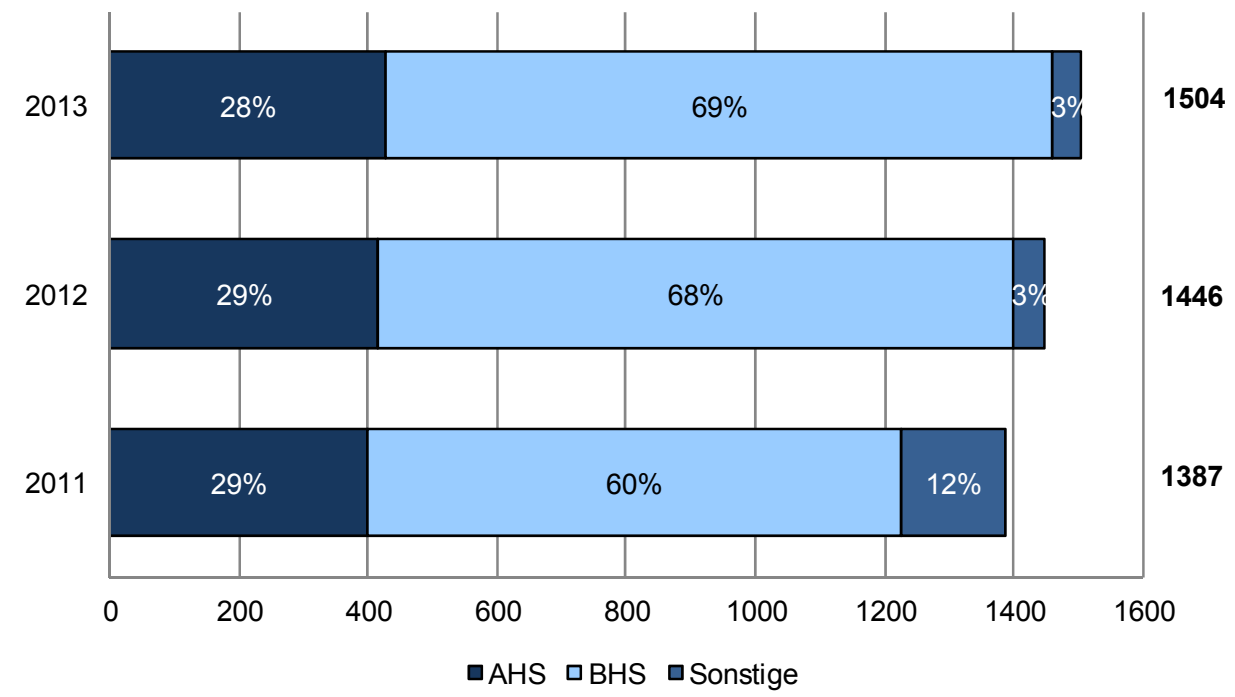

Quelle: FFG-Monitoring-Daten

\section{Grafik 13 Anspruchsgehalt des Praktikums}

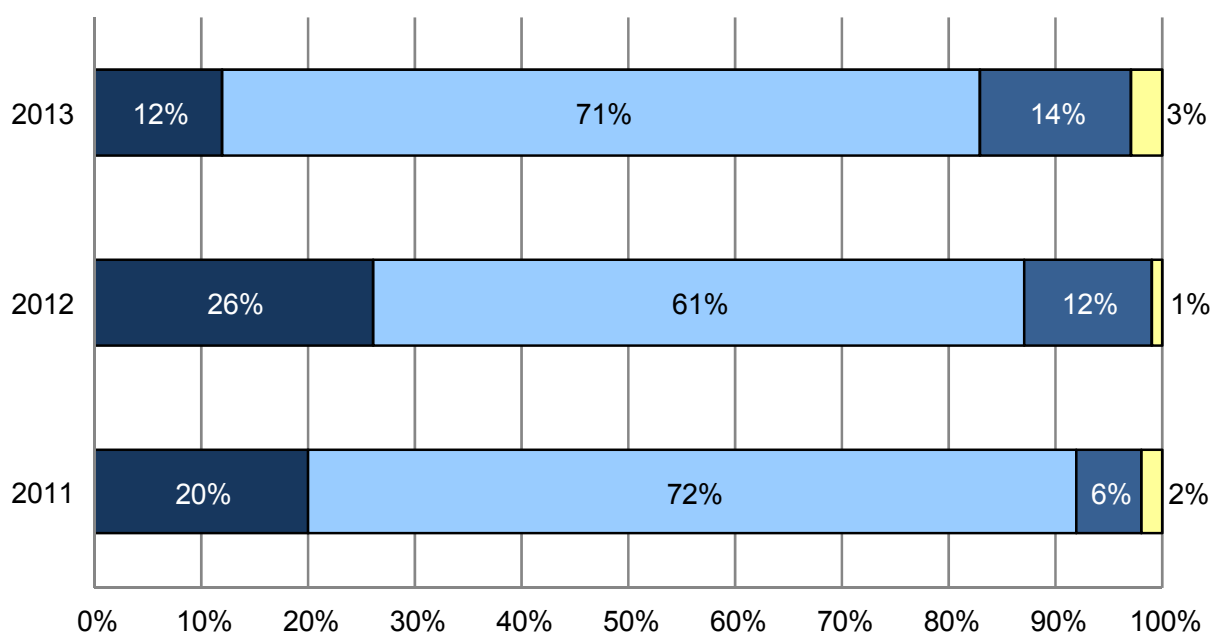

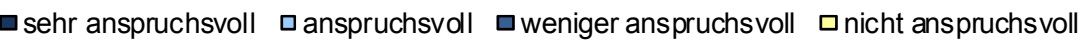

Quelle: Talente entdecken: Praktika für Schülerinnen und Schüler, Feedback SchülerInnen; n=133 (2011), 164 (2012), 187 (2013) 


\section{Talente regional}

Grafik 14 Initiative zur Teilnahme am Projekt

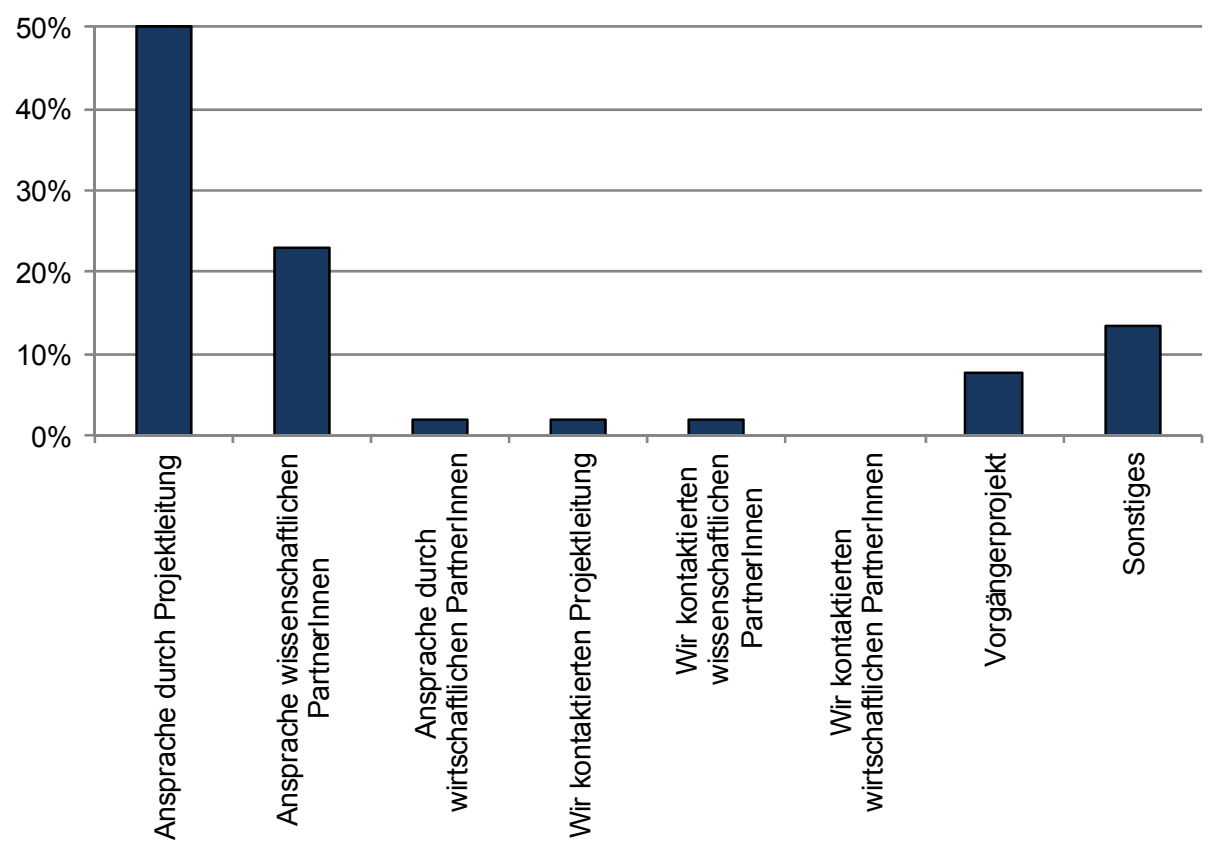

Quelle: Online-Befragung der in Talente regional involvierten Pädagoglnnen, $\mathrm{n}=52$

\section{Grafik 15 Kenntnis der Programmlinie Talente regional}

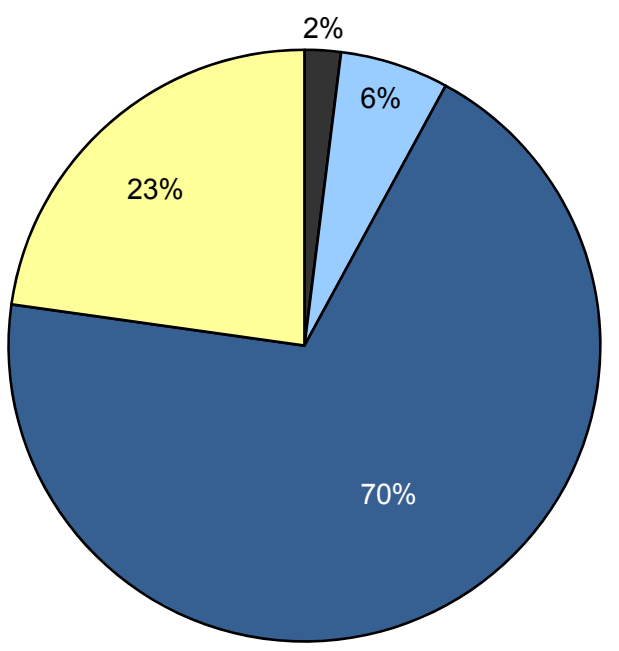

- Messebesuch

口Science Center Netzwerk

口Persönliche Kontakte

口Sonstiges

Quelle: Online-Befragung der in Talente regional involvierten Pädagoglnnen, $n=52$ 


\section{Grafik 16 Früherer Kontakt zu ProjektpartnerInnen}

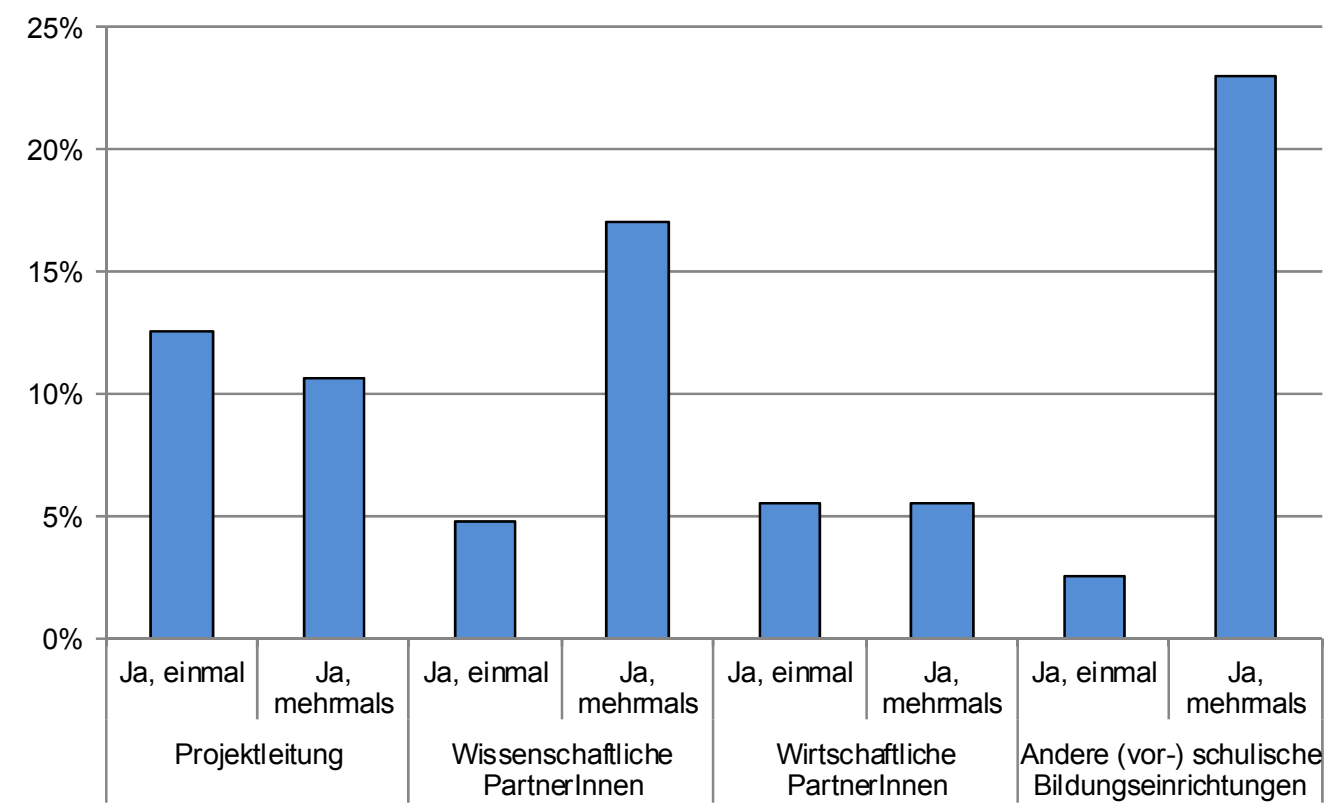

Quelle: Online-Befragung der in Talente regional involvierten PädagogInnen, n=36

\section{Grafik 17 Wirkungen bei den teilnehmenden Kindern/Jugendlichen}

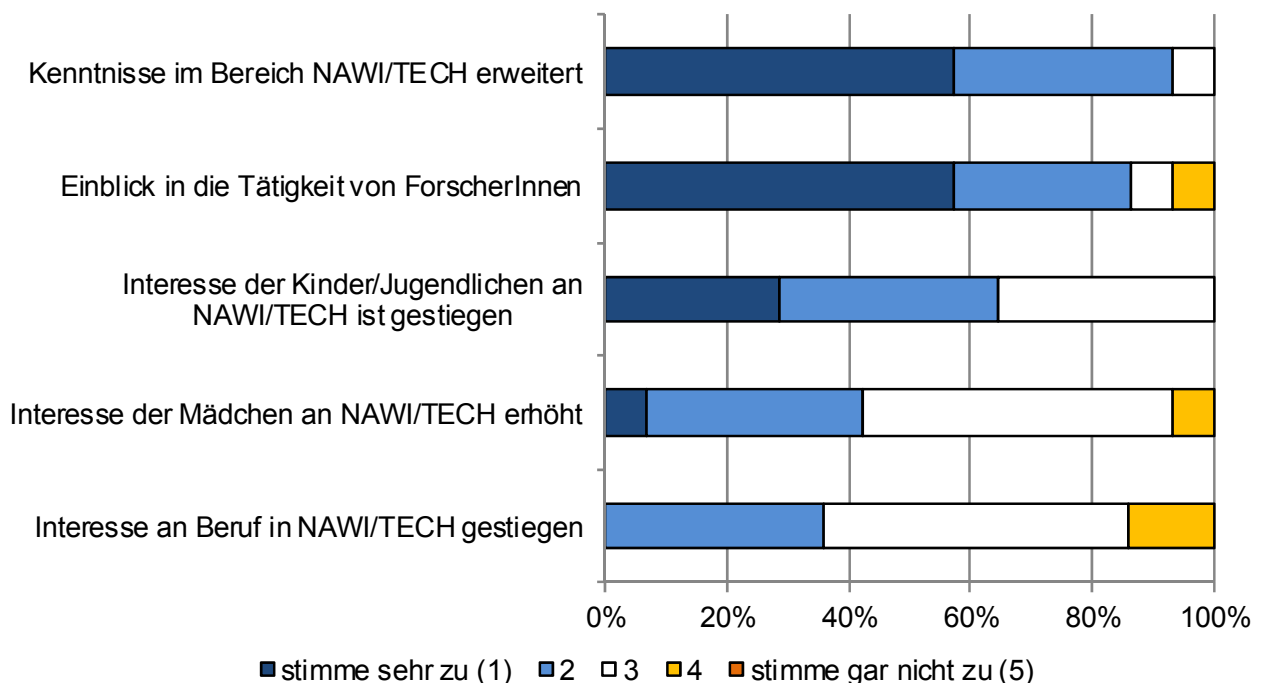

Quelle: Online-Befragung der in Talente regional involvierten PädagogInnen, n=14 
Grafik 18 Wirkungen bei den PädagogInnen

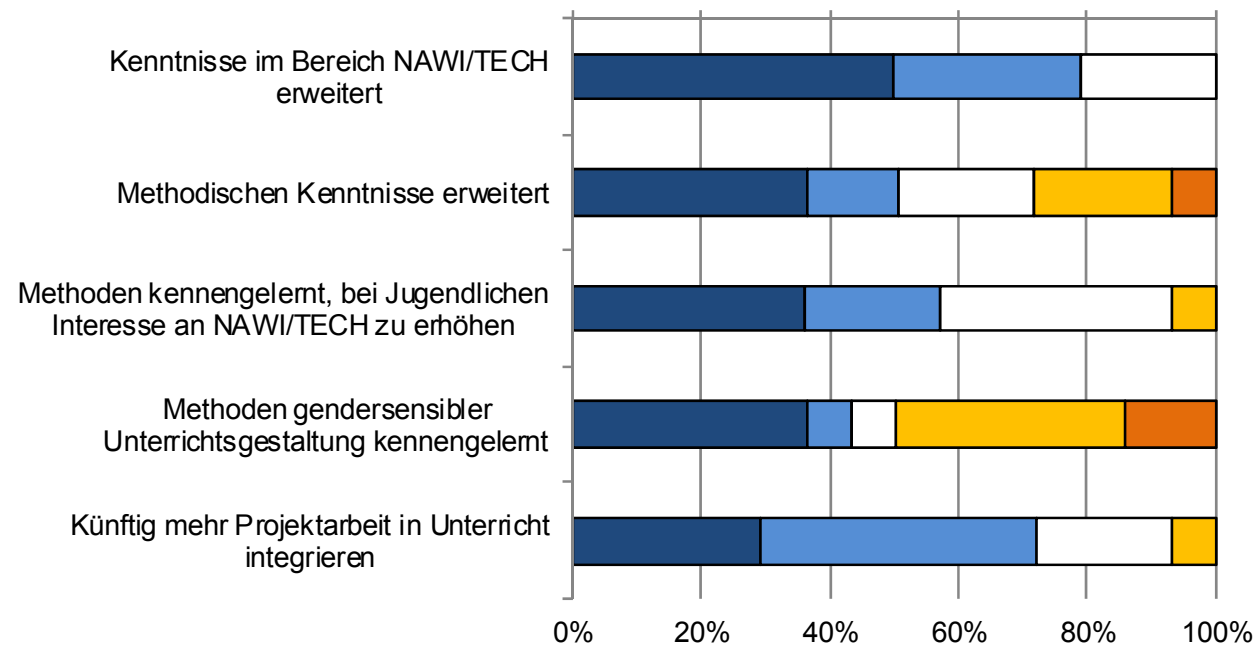

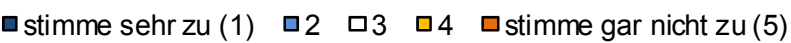

Quelle: Online-Befragung der in Talente regional involvierten PädagogInnen, $n=14$

\section{FEMtech Praktika für Studentinnen}

Grafik 19 Praktikumsdauer der FEMtech Praktika für Studentinnen

2. Ausschreibung

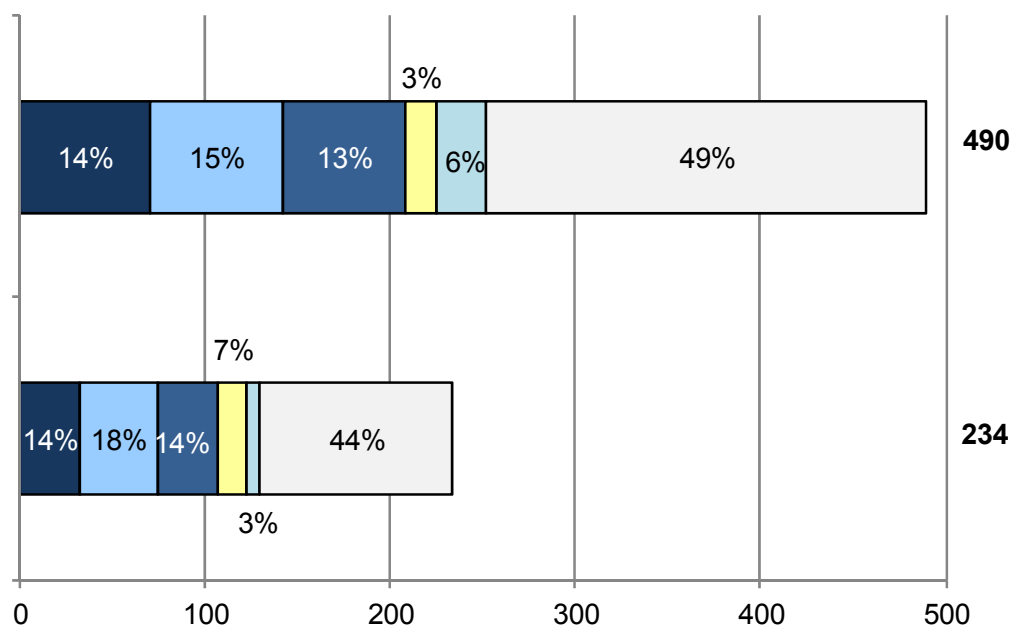

घ1 Monat $\square 2$ Monate $\square 3$ Monate $\square 4$ Monate $\square 5$ Monate $\square 6$ Monate

Quelle: Praktika für Studentinnen, Detailstatistik: geförderte Praktika 


\section{Gesamtbetrachtung}

Grafik 20 Verteilung der Organisationstypen und Projektteilnahmen

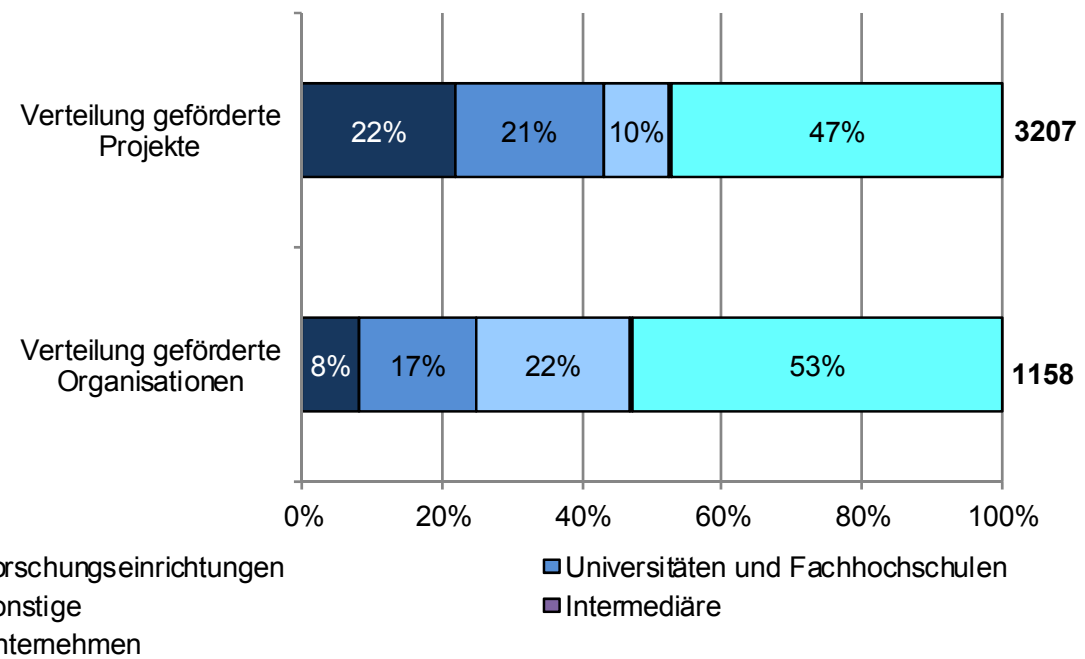

Quelle: FFG Monitoringdaten

\section{Grafik 21 Verteilung Erstförderungen}

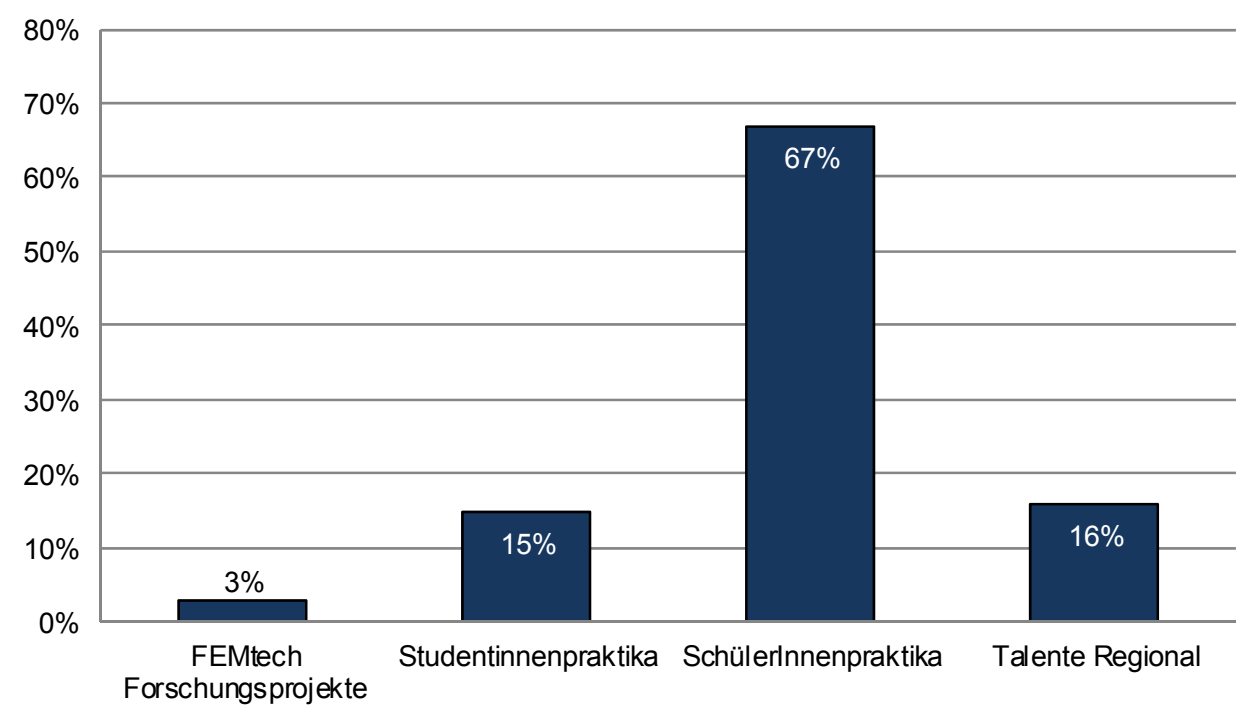

Quelle: FFG Monitoringdaten, n=157 (exklusive Karriere Grants) 
KMU Forschung Austria

Austrian Institute for SME Research 